RESISTANCE EXERCISE TIMING AND

METABOLIC RISK FACTORS IN TYPE 2 DIABETICS

A Dissertation
presented to
the Faculty of the Graduate School
at the University of Missouri -Columbia
In Partial Fulfillment
of the Requirements for the Degree
Doctor of Philosophy
by
TIMOTHY D. HEDEN
Dr. Jill A. Kanaley, Dissertation Advisor
JULY 2014


The undersigned, appointed by the Dean of the Graduate School, have examined the dissertation entitled

RESISTANCE EXERCISE TIMING AND

\section{METABOLIC RISK FACTORS IN TYPE 2 DIABETICS}

Presented by Timothy D. Heden,

a candidate for the degree of doctor of philosophy,

and herby certify that, in their opinion, it is worthy of acceptance

Professor Jill A. Kanaley

Assistant Professor R. Scott Rector

Associate Professor John P. Thyfault

Professor Frank W. Booth 


\section{ACKNOWLEDGEMENTS}

I would like to thank Jill Kanaley for her guidance and support over the past several years. I have acquired a great deal of knowledge and skills working in her lab, which helped me land a great post doc job. I thank Nathan Winn for helping with blood draws and blood processing during the exercise study days for my Dissertation project and with numerous other projects in the lab. I thank Ying Liu for placing the catheters, helping with blood draws and blood processing, and teaching me how to perform several different assays in the lab. I thank Andrea Mari for his assistance with the beta-cell function modeling and interpretation of the data. I thank John Thyfault for serving on my dissertation committee and for allowing me the privilege to work in his lab for a brief period of time to perform a pre-clinical animal study. I thank Frank Booth for serving on my dissertation committee and teaching me how to train rats on a treadmill. I thank Scott Rector for serving on my dissertation committee. I also thank Dr. Tom Thomas for his guidance during my first couple of years in the program at MU. 


\section{TABLE OF CONTENTS}

ACKNOWLEDGEMENTS .......................................................

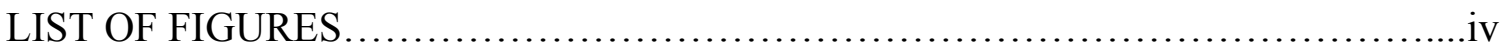

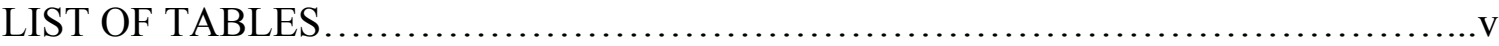

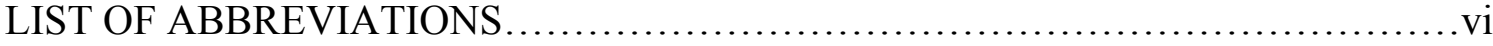

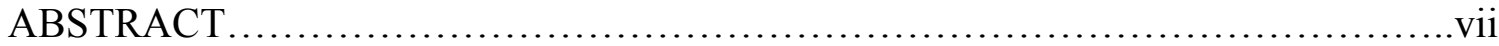

CHAPTER:

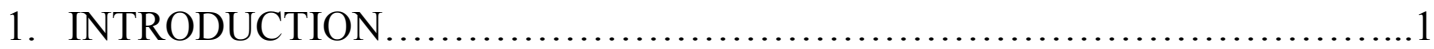

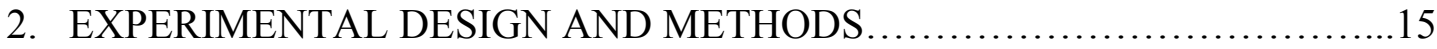

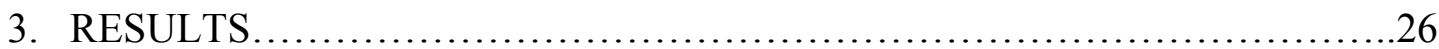

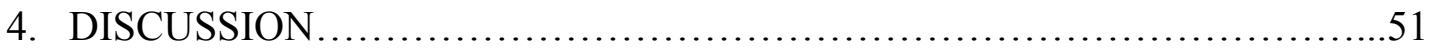

5. EXTENDED LITERATURE REVIEW .................................64

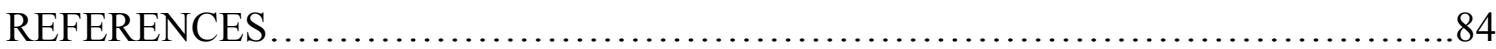

\section{APPENDIX:}

A. Informed Consent.................................................. 96

B. Manuscript...................................................... 103

C. Curriculum Vitae....................................................... 133

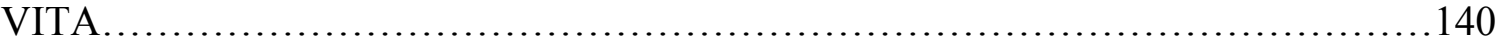




\section{LIST OF FIGURES}

Figure

1. U.S. Population Trends with Diagnosed Diabetes................................

2. Study Design For Specific Aim 1, 2, and 3.................................... 18

3. Blood Glucose Concentrations During Mixed Meal Testing $\ldots . . \ldots \ldots \ldots \ldots \ldots \ldots \ldots . . .28$

4. Change in Glucose iAUC During Each Exercise Trial In Each Subject...............29

5. Total Triacylglycerol Concentrations During Mixed Meal Testing...................30

6. Chylomicron Triacylglycerol Concentrations During Mixed Meal Testing............31

7. VLDL-1 Triacylglycerol Concentrations During Mixed Meal Testing................32

8. VLDL-2 Triacylglycerol Concentrations During Mixed Meal Testing................33

9. Change in Triacylglycerol iAUC During Each Exercise Trial In Each Subject ........34

10. Free Fatty Acid Concentrations During Mixed Meal Testing......................35

11. Insulin Concentrations During Mixed Meal Testing ............................. 36

12. C-peptide Concentrations During Mixed Meal Testing...........................37

13. Insulin Secretion Rates During Mixed Meal Testing ........................... 38

14. Insulin Clearance During Mixed Meal Testing.................................39

15. Glucagon Concentrations During Mixed Meal Testing..........................40

16. GIP Concentrations During Mixed Meal Testing.............................41

17. GLP-1 Concentrations During Mixed Meal Testing ...........................42

18. Acetaminophen Concentrations (Gastric Emptying) During Mixed Meal Testing...43

19. Subjective Well-being During Mixed Meal Testing..............................46

20. Nocturnal Blood Glucose Concentrations After Testing In The Lab...............47

21. Postprandial Glucose Concentrations After Breakfast..........................48

22. Blood Glucose Concentrations Over 24 Hour Period..............................49 


\section{LIST OF TABLES}

Table

1. Clinical Criteria for Normal, Pre-diabetic, and Diabetic Individuals.................

2. Hypothesized $4 \mathrm{~h}$ postprandial responses during each trial......................... 14

3. Participant Characteristics................................................ 26

4. Metabolic Data And Rating of Perceived Exertion During Exercise.................27

5. Energy Expenditure and Substrate Oxidation $~ 3.5$ - 4 h After Dinner Meal..........44

6. Beta-cell Function Parameters..............................................44

7. Nocturnal and Post-breakfast Glucose Responses..............................48

8. Average Blood Glucose and Glycemic Variability Over 24 h.....................50

9. Morning Fasting Measures The Day After Testing In The Lab....................50 


\section{LIST OF ABBREVIATIONS}

1. Aerobic Exercise (AE)

2. Resistance Exercise (RE)

3. No Resistance Exercise (NoRE)

4. Pre-dinner Resistance Exercise $(\mathrm{RE} \rightarrow \mathrm{M})$

5. Post-dinner Resistance Exercise $(\mathrm{M} \rightarrow \mathrm{RE})$

6. Type 2 Diabetes (T2D)

7. Incremental area under the curve (iAUC)

8. Free Fatty Acids (FFA)

9. Glucose-dependent Insulinotropic Polypeptide (GIP)

10. Glucagon-like Peptide-1 (GLP-1)

11. Triacylglycerol (TAG)

12. Very-low Density Lipoproteins (VLDL)

13. Glycated hemoglobin (HbA1c)

14. Continuous Glucose Monitor System (CGMS)

15. 10 Repetition Maximum (10-RM)

16. Respiratory Exchange Ratio (RER)

17. Quantitative Insulin Sensitivity Check Index (QUICKI)

18. Homeostatic Model Assessment of Insulin Resistance (HOMA-IR) 


\title{
RESISTANCE EXERCISE TIMING AND \\ METABOLIC RISK FACTORS IN TYPE 2 DIABETICS
}

Timothy D. Heden

Dr. Jill A. Kanaley, Dissertation Supervisor

\begin{abstract}
Abnormally elevated postprandial glucose and triacylglycerol (TAG) concentrations are risk factors for cardiovascular disease in patients with type-2 diabetes. The most effective time to exercise to lower postprandial glucose and TAG concentrations is unknown. Thus, the aim of this study was to determine what time is more effective, either pre- or post-dinner resistance exercise (RE), at improving postprandial risk factors in patients with type-2 diabetes. Methods: Thirteen obese patients with type 2 diabetes completed three trials in a random order in which they consumed a standardized dinner meal with 1) no $\mathrm{RE}(\mathrm{NoRE}), 2)$ pre-dinner $\mathrm{RE}(\mathrm{RE} \rightarrow \mathrm{M})$, and 3) post-dinner RE beginning $45 \mathrm{~min}$ after dinner $(\mathrm{M} \rightarrow \mathrm{RE})$. During each trial blood samples were taken to measure glucose, TAG, acetaminophen (gastric emptying), endocrine responses, and mathematical modeling was used to asses beta-cell function. Indirect calorimetry was used to measure energy expenditure and substrate oxidation. A continuous glucose monitor was used to assess nocturnal and morning glycemic control the next day. A fasting blood sample was taken the following morning and the quantitative insulin sensitivity check index (QUICKI) was used to estimate whole body insulin sensitivity and the homeostatic model assessment of insulin resistance (HOMA-IR) was used as an estimate of hepatic insulin resistance. Results: The postprandial glucose iAUC was reduced $(\mathrm{P}<0.05)$ by $\sim 18 \%$ and $30 \%$ during the $\mathrm{RE} \rightarrow \mathrm{M}$ and $\mathrm{M} \rightarrow \mathrm{RE}$ trials, respectfully, compared to NoRE, with no
\end{abstract}


difference between RE trials. The postprandial total TAG iAUC was $\sim 92 \%$ lower (P $<$ 0.05 ) during $\mathrm{M} \rightarrow \mathrm{RE}$ compared to NoRE and $\mathrm{RE} \rightarrow \mathrm{M}$, an effect due to lower VLDL-1 TAG concentrations. $\mathrm{RE} \rightarrow \mathrm{M}$ and $\mathrm{M} \rightarrow \mathrm{RE}$ reduced the insulin $i \mathrm{AUC}$ by $35 \%$ and $48 \%$, respectfully, compared to NoRE $(\mathrm{P}<0.05)$, but via different mechanisms as $\mathrm{RE} \rightarrow \mathrm{M}$ enhanced insulin clearance, whereas $\mathrm{M} \rightarrow \mathrm{RE}$ reduced pancreatic insulin secretion and enhanced insulin clearance. The postprandial GLP-1 iAUC was $\sim 49 \%$ lower $(\mathrm{P}<0.05)$ during $\mathrm{M} \rightarrow \mathrm{RE}$ compared to NoRE and $\mathrm{RE} \rightarrow \mathrm{M}$. Gastric emptying, beta-cell function, postprandial substrate oxidation, GIP, nocturnal and morning glycemic control, and the QUICKI and HOMA-IR index the next morning were not significantly different between trials $(\mathrm{P}>0.05)$. Conclusions: Post-dinner RE improves metabolic risk factors more effectively than pre-dinner RE in obese patients with type 2 diabetes. However, the acute metabolic health benefits of RE are short-lived and do not extend into the overnight or morning period. 


\section{CHAPTER 1 - INTRODUCTION}

Type 2 diabetes (T2D) is the most common form of diabetes mellitus and can be characterized by fasting and postprandial hyperglycemia. Clinically, T2D is diagnosed when an individual has a glycosylated hemoglobin (HbAlc) level $\geq 6.5 \%$, a fasting plasma glucose $\geq 126 \mathrm{mg} / \mathrm{dL}$ (fasting defined as no caloric intake for at least $8 \mathrm{~h}$ ), or a plasma glucose $\geq 200 \mathrm{mg} / \mathrm{dL}$ at $2 \mathrm{~h}$ post an oral glucose tolerance test (Table 1).

Table 1. Clinical criteria for normal, pre-diabetic, and diabetic individuals.

\begin{tabular}{lccc}
\hline & HbAlc $(\%)$ & $\begin{array}{c}\text { Fasting Plasma Glucose } \\
(\mathrm{mg} / \mathrm{dL})\end{array}$ & $\begin{array}{c}\text { Two-hour Oral Glucose } \\
\text { Tolerance Test }(\mathrm{mg} / \mathrm{dL})\end{array}$ \\
\hline Diabetes & $\geq 6.5$ & $\geq 126$ & $\geq 200$ \\
Pre-Diabetes & 5.7 to 6.4 & 100 to 125 & 140 to 199 \\
Normal & $\leq 5.6$ & $\leq 99$ & $\leq 139$ \\
\hline
\end{tabular}

Source: [1]

The prevalence of T2D is increasing in the U.S. [2-3] and developed nations around the world [4-5]. In the U.S., over 21 million Americans ( $7 \%$ of the population) have been diagnosed with diabetes [6] (Figure 1).

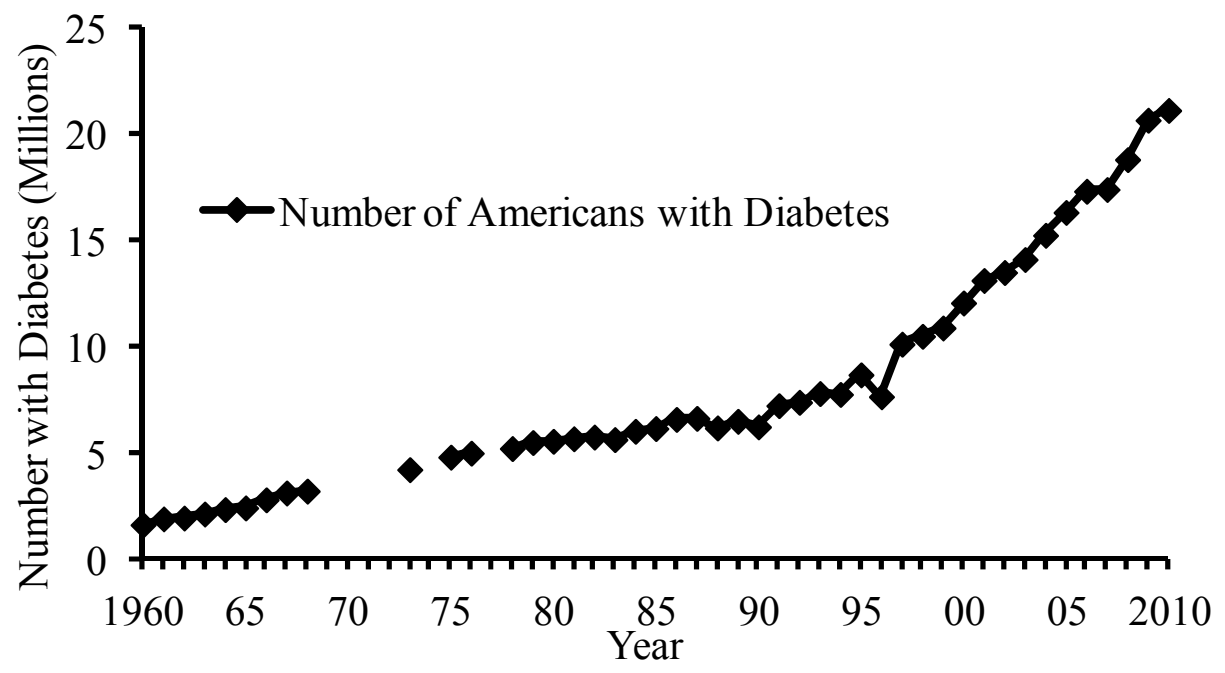

Figure 1. U.S. population trends with diagnosed diabetes [6]. 
According to the World Health Organization, diabetes is the $7^{\text {th }}$ leading cause of death in developed nations [7]. In addition, T2D provokes the development of several other diseases including vascular (macro and micro) disease (the leading cause of death in developed nations), stroke, hypertension, blindness, kidney disease, nervous system disease, and if untreated can lead to amputations [8]. In addition, the health care costs of treating T2D and its co-morbidities are staggering and place a heavy burden on healthcare budgets [9-12]. Thus, it is critical that effective therapies be identified that prevent or treat $\mathrm{T} 2 \mathrm{D}$. In the treatment of $\mathrm{T} 2 \mathrm{D}$, the main goal is to increase insulin sensitivity and attenuate hyperglycemia and hypertriacylglyceridemia, which can be accomplished with bariatric surgery, pharmacologically, and/or with lifestyle intervention. In particular, exercise is effective at improving metabolic control in individuals with $\mathrm{T} 2 \mathrm{D}$, and thus is a cornerstone in the treatment of T2D.

\section{Exercise Guidelines for Type 2 Diabetics}

Numerous studies show that aerobic exercise (AE) training, resistance exercise (RE) training, or a combination of both improves glycemic control or prevents glycemic control from worsening in individuals with T2D [13-31]. Further, combination training has been shown to be more effective than either one alone in improving glycemic control [13-14]. Thus, the current exercise guidelines for individuals with T2D provided by the American College of Sports Medicine and American Diabetes Association state individuals with T2D should partake in at least $150 \mathrm{~min} /$ week of moderate to vigorous AE spread out over $3 \mathrm{~d}$ per week and engage in moderate to vigorous RE at least 2-3 d per week [32-34]. Although acute exercise or exercise training generally improves glycemic and lipemic control, inter-individual variability is large as there is considerable 
heterogeneity in responses [35-37], and there are even a couple of studies demonstrating a single session of exercise has no significant impact on glycemic [38-39] or lipemic [37, 40] control in individuals with T2D. It is not completely clear why some individuals are "exercise sensitive" and have beneficial changes in glycemia and/or lipemia with exercise and some individuals are "exercise insensitive" and do not experience improvements or have a worsening of glycemic or lipemic control with exercise. Possible explanations for this phenomenon after exercise training could be genetic factors [41] or baseline (pretraining) fasting blood glucose concentrations [42-43]. Following an acute session of exercise, a lack of change in glycemia with exercise has been related to $\mathrm{HbAlc}$, such that individuals with a greater HbA1c (thus higher glucose concentrations) have bigger improvements in glycemic control following an acute AE session, whereas individuals with more tightly controlled glycemic control (lower HbAlc concentrations) have less of an improvement [36]. In another study, an acute session of AE prior to a meal did not result in a significant change in postprandial TAG concentrations on average, but there was tremendous inter-individual variability in the response with some individuals having improvements, while others had no improvement or the postprandial TAG response increased. Interestingly, in this study the postprandial TAG response was related to the propensity to increase hepatic fatty acid oxidation following $\mathrm{AE}$, such that individuals that had greater increases in hepatic fatty acid oxidation following $\mathrm{AE}$ had lower postprandial TAG concentrations [37]. Another possibility that has been overlooked is the timing of exercise around a meal. An unexamined hypothesis is that, and in the context of acute exercise, some individuals are exercise insensitive (non-responders) because they are exercising at an inappropriate time relative to a meal, and that moving 
the exercise session from before a meal to after a meal may perhaps make an individual exercise sensitive (responder). Currently, the exercise guidelines for individuals with T2D only include the recommended mode, dosage, frequency, and intensity, but do not include any information on the best time to exercise, particularly relative to the last meal (before or after), to improve metabolic risk factors including postprandial glucose and TAG concentrations. With a push toward personalized medicine, an understanding of the best time to exercise to improve glycemic and lipemic control is warranted so that more effective exercise prescriptions can be made.

\section{Exercise Timing and Postprandial Glycemia and Lipemia in Type 2 Diabetics}

The few studies that have directly examined how exercise timing impacts glycemic and lipemic control are acute exercise studies utilizing AE. The first series of studies highlighting the potential importance of exercise timing were done by Poirier and colleagues [44-46]. In one study, capillary blood glucose was measured before and after 1 $\mathrm{h}$ of moderate intensity cycling $\left(60 \%\right.$ of $\left.\mathrm{VO}_{2} \max \right)$ either in the fasted state or after a meal (within 5 h). Exercise in the fasting state did not change blood glucose levels, whereas post-meal exercise within $5 \mathrm{~h}$ of the last meal reduced blood glucose considerably (between $28-43 \%$ decrease) [45]. Using a similar study design, Gaudet-Savard et al. [44] showed that exercise in the fasted state resulted in variable changes in blood glucose depending on the pre-exercise glucose level such that individuals with pre-exercise blood glucose levels $<6 \mathrm{mmol} / 1$ would experience an increase in blood glucose during exercise, while individuals with pre-exercise blood glucose levels $>6 \mathrm{mmol} / \mathrm{l}$ would experience no change or a decrease in blood glucose during exercise. On the contrary, the response was more uniform with post-meal exercise, as exercise in the fed state reduced blood glucose 
concentrations in all individuals regardless of the pre-exercise blood glucose level, although the glucose lowering effect of exercise was more pronounced in individuals with higher blood glucose concentrations. In addition, post-meal exercise lowered blood glucose the most when performed within $5 \mathrm{~h}$ of the last meal. A limitation of these studies is the lack of stringent dietary control, blood glucose was only measured before and after exercise, and there was no direct comparison of pre-meal vs. post-meal exercise. In a more carefully controlled study, $1 \mathrm{~h}$ of moderate intensity $\mathrm{AE}\left(60 \%\right.$ of $\mathrm{VO}_{2}$ max $)$ performed after breakfast in the lab (starting $2 \mathrm{~h}$ after breakfast) lowered blood glucose concentrations more so than exercise in the fasted state [46]. This study is limited because there was no direct comparison of pre-meal vs. post-meal exercise on postprandial glucose responses. To my knowledge, the only study to directly compare the effect of pre-meal AE against post-meal AE was done by Colberg et al. [47], and they found that 20 min of self-paced treadmill walking performed 20 min after a dinner meal significantly lowered postprandial blood glucose concentrations, but the same bout of exercise performed prior to dinner had no effect on postprandial blood glucose concentrations. Taken together, these data indicate that post-meal AE has more of a beneficial impact on postprandial glycemia than postabsorptive $\mathrm{AE}$ in individuals with T2D.

Only three studies have examined the impact of acute $\mathrm{AE}$ on postprandial triacylglycerol (TAG) concentrations in individuals with T2D. In two of the studies [37, 40], the $\mathrm{AE}$ bout was performed the day prior to the test meal given, and these studies reported that on average prior AE had no effect on postprandial TAG concentrations, compared to a no exercise trial, although the response was highly variable. Interestingly, 
in another study the AE bout was performed starting 90 min after a high fat breakfast meal, and in this study the exercise bout significantly reduced the average postprandial TAG response in individuals with T2D [48]. Although no study has directly compared pre-meal exercise to post-meal exercise, the available research suggests that the timing of $\mathrm{AE}$ around a meal might be critical in lowering postprandial TAG concentrations. It is possible that changing exercise timing from pre-meal to post-meal may make an individual more exercise sensitive, although this hypothesis has never been examined.

The aforementioned studies cited examined AE, but no study has examined how resistance exercise (RE) timing relative to dinner alters postprandial glycemia and lipemia. From a practical perspective many obese type 2 diabetic patients may not enjoy $\mathrm{AE}$ or be motivated to perform $\mathrm{AE}$ soon after a meal, but $\mathrm{RE}$ may be a practical alternative and may be preferred over $\mathrm{AE}$ in some obese individuals because traditional $\mathrm{RE}$ is performed with the person sitting or lying down and there are short work periods with long rests between sets. However, compared to AE, RE is a very different physiological stimulus (i.e. RE is anaerobic, does not involve continuous skeletal muscle contractions, and has lower energy expenditure), thus it is not clear if RE would improve postprandial glycemia and lipemia as robustly as AE. Some studies report acute RE prior to a meal improves glycemic control [49-50], while other studies report it does not [38], and no study has assessed how acute RE alters postprandial TAG concentrations. In addition, these studies assessed the effects of RE on postprandial glycemia and lipemia after a breakfast or lunch meal, and not a dinner meal later in the day, a time when postprandial glucose and TAG concentrations have been reported to be highest in individuals with T2D [51-52]. Thus, more research is needed to validate RE as an 
effective means to improve postprandial glucose and TAG concentrations. Further, the best time to perform RE in obese patients with T2D needs to be established. Knowledge of the best time to perform RE will help health care professionals design more personalized exercise programs that maximize the metabolic benefits of exercise. This information is of clinical interest and will provide translational knowledge that can be used to make better exercise prescriptions and exercise guidelines for individuals with T2D.

\section{Why Might Post-meal Exercise Be Better at Improving Glycemic and Lipemic Control?}

There are several potential mechanisms explaining why post-meal exercise may be superior at lowering postprandial glucose than pre-meal exercise in individuals with T2D. Although both pre- and post-meal exercise may increase skeletal muscle glucose uptake, post-meal exercise may increase skeletal muscle glucose uptake more. This premise is supported by studies by Larsen et al. [53-54] that illustrated that compared to a no exercise trial, moderate or high intensity AE performed 45 min after a breakfast meal increased the rate of disappearance of the infused tracer $3-\left[{ }^{3} \mathrm{H}\right]$-glucose (labeling endogenous glucose) without altering its rate of appearance. In addition, the postprandial glucose response to a subsequent lunch meal was unaltered in this study, which further supports the concept that post-meal exercise is better compared to pre-meal exercise at lowering postprandial glucose concentrations. Since individuals with T2D (non-insulin dependent) have some insulin secretion [53-54], it is feasible that post-meal exercise increases skeletal muscle glucose uptake to a greater extent compared to pre-meal exercise because of the synergistic effect of insulin and skeletal muscle contractions on increasing skeletal muscle glucose uptake in addition to insulin suppressing endogenous 
glucose output from the liver. After ingestion of a mixed meal, the insulin/glucagon ratio increases, which results in two simultaneous effects including 1) attenuated hepatic glucose output [55] and 2) increases in skeletal muscle blood flow and capillary recruitment [56], glucose transporter type 4 (GLUT-4) translocation [57], and blood glucose uptake [58]. Although these processes are impaired in individuals with T2D, the effect of insulin on skeletal muscle blood glucose uptake is enhanced by skeletal muscle contractions, as data has shown insulin [57] and muscle contractions [57, 59] independently increase skeletal muscle GLUT-4 translocation and blood glucose uptake, and when combined synergistically increase skeletal muscle GLUT-4 translocation and/or blood glucose uptake to a greater extent than either one alone in humans [57] and rodents [60-61]. Thus, postprandial exercise in the face of increasing insulin concentrations with a prior meal should synergistically increase skeletal muscle blood glucose uptake and suppress hepatic glucose output, and result in lower postprandial blood glucose compared to pre-meal exercise.

Additionally, reduced gastric emptying may work in conjunction with increased skeletal muscle blood glucose uptake and reduced hepatic glucose output to lower postprandial blood glucose concentrations with post-meal exercise, but not with pre-meal exercise. Prior research has shown that postprandial AE augments the postprandial glucagon-like peptide-1 (GLP-1) response in healthy individuals, an effect that was associated with lower blood glucose concentrations [62]. One function of GLP-1 is to inhibit gastric emptying [63-65]. High intensity AE has been shown to reduce gastric emptying of a carbohydrate solution in healthy men [66-67], thus it is possible that the reduced gastric emptying caused by higher intensity AE is mediated by higher GLP-1 
concentrations (although this has never been examined experimentally). The effect that RE has on GLP-1 or gastric emptying in individuals with T2D is unknown. However, it is plausible that post-meal RE would augment postprandial GLP-1 concentrations, and this would be associated with reduced gastric emptying and reduced glucagon concentrations (as GLP-1 has also been shown to inhibit glucagon release from the pancreas), an effect that will not be present with pre-meal RE. Ultimately, a reduced rate of gastric emptying will reduce glucose delivery to the intestine, which will reduce glucose dependent insulinotropic polypeptide (GIP) secretion from the intestine, and glucose uptake into the blood stream, thereby resulting in lower postprandial blood glucose, insulin, and cpeptide concentrations with post-meal RE compared to pre-meal RE.

The available research suggests that post-meal AE reduces postprandial TAG concentrations [48], while pre-meal AE does not in patients with type 2 diabetes [37, 40]. This effect may be mediated by the interplay of a couple of different mechanisms involving increased clearance of TAG and/or reduced secretion of TAG. During postmeal exercise, circulating free fatty acid concentrations are low (because the meal increases insulin concentrations, and insulin reduces lipolysis) so there may not be enough free fatty acids to supply the increase in skeletal muscle fatty acid oxidation induced by exercise, thus more TAG are hydrolyzed so that free fatty acids from TAG can be liberated and taken up from the blood by the exercising skeletal muscle. In addition, post-meal exercise likely increases skeletal muscle blood flow, which increases blood flow through previously non-flowing capillaries, allowing more TAG to be exposed to lipoprotein lipase (enzyme that hydrolyzes TAG to glycerol and free fatty acids) which would produce more widespread hydrolysis of TAG. 
Another possibility is that post-meal exercise reduces endogenous and exogenous

TAG secretion. Post-meal exercise may reduce gastric emptying, which will slow the digestion and absorption of dietary fatty acids and thus reduce the secretion of exogenous chylomicron TAG from the intestine. It is also possible post-meal exercise reduces endogenous very-low density lipoprotein TAG (VLDL-TAG) secretion from the liver perhaps by changing gut blood flow, hormones/metabolites concentrations delivered to the liver, or neural input to the liver. All of these mechanisms are speculative and warrant further investigation.

\section{Acute Resistance Exercise and Nocturnal and Fasting Blood Glucose}

Although many individuals with T2D spend the majority of their day in the postprandial state during the day, a significant portion of their day is during the overnight period, and the lowering of nocturnal blood glucose concentrations is of clinical interest and an important component in the treatment of T2D. Only two studies have directly examined how acute RE impacts nocturnal blood glucose concentrations, and both studies used continuous glucose monitoring (CGM) technology. van Dijk et al. [50] reported that an acute bout of $\mathrm{RE}$ in the morning prior to lunch reduced the prevalence of nocturnal (12-6 a.m.) hyperglycemia (defined as time spent with blood glucose $>180$ $\mathrm{mg} / \mathrm{dL}$ ) the following night but did not significantly alter the average glucose concentration [50]. In contrast, Bacchi et al. [39] reported that an acute bout of RE at night prior to dinner ( 6:30 p.m.) did not alter nocturnal $(1-5$ a.m.) average blood glucose concentrations. In addition, some [49, 68], but not all [69] research has demonstrated that acute RE does not improve insulin sensitivity or lowers fasting blood glucose concentrations the next morning in individuals with T2D or impaired fasting 
blood glucose concentrations. Based on the majority of these studies, it appears that premeal exercise prior to lunch or dinner does not alter subsequent nocturnal blood glucose concentrations or insulin sensitivity the next day. It is not known if performing RE after dinner, a time that is closer to the nocturnal period, would have a more significant impact on lowering nocturnal blood glucose concentrations.

\section{Summary}

In summary, T2D places a large burden on an individual's health and well-being in addition to the health care system [9-12], and thus cost friendly and effective treatment strategies are needed to reduce these burdens. Overall, exercise improves glycemic and lipemic control in individuals with T2D, but most studies show great variability in the response with some individuals being exercise sensitive (i.e. have improvements in glycemic and lipemic control with acute exercise, responders) while others are exercise insensitive (i.e. do not have improvements in glycemic or lipemic control with acute exercise, non-responders) [35-37]. It is possible that some individuals are not sensitive to exercise because they are exercising at an inappropriate time, but the most optimal time to exercise is not clear. Although limited, some data suggests that post-meal AE is more beneficial at improving postprandial glucose and TAG concentrations than pre-meal AE in individuals with T2D [47-48]. The superior effect of post-meal AE on lowering postprandial blood glucose and TAG concentrations may be partially mediated by reduced gastric emptying and gut blood flow, although the relationship between different exercise times and changes in gastric emptying and postprandial glycemia and lipemia has never been studied. The impact that RE timing (pre-meal RE vs. post-meal RE) has on postprandial or nocturnal glycemic or lipemic control in individuals with T2D has 
never been studied. Obese individuals with $\mathrm{T} 2 \mathrm{D}$ may not enjoy AE or be motivated to perform $\mathrm{AE}$ soon after a meal, thus $\mathrm{RE}$ is an alternative option and may be preferred over $\mathrm{AE}$ in obese individuals. Compared to $\mathrm{AE}, \mathrm{RE}$ is a very different physiological stimulus (i.e. RE is anaerobic, recruits more type II skeletal muscle fibers, and is not continuous), thus it is not clear if post-meal RE would improve postprandial glucose and TAG concentrations as robustly as AE. Further, little research has examined how a single RE session alters glycemic control in individuals with $\mathrm{T} 2 \mathrm{D}$, and the data are mixed as some studies report RE improves glycemic control in individuals with T2D [49-50] while other studies report it does not [38]. No study has assessed how a single session of RE alters postprandial TAG concentrations in individuals with T2D. Therefore, more research is needed to validate $\mathrm{RE}$ as an effective means to improve metabolic control in the evening and examine how RE timing relative to a dinner meal impacts post-meal metabolic control to identify the best time to exercise in obese individuals with T2D. Knowledge of the best time to exercise will allow health care practitioners to make more effective exercise prescriptions that maximize the metabolic benefits of RE and reduce exercise non-responders.

\section{Specific Aims, Hypotheses, Delimitations, Significance, and Innovation}

The overarching goal of this dissertation project was to determine if performing RE after a meal is better at improving metabolic control (possibly by reducing gastric emptying), compared to pre-meal RE, in individuals with T2D. Importantly, another goal of this project was to determine how the different RE times altered subjective well-being (pleasure) to determine how feasible each RE time is (i.e. did the participants actually 
enjoy exercising before or after they ate compared to not exercising). The specific aims of this project are:

Specific Aim 1: To determine if post-dinner RE is more effective compared to pre-dinner $\mathrm{RE}$ at improving postprandial glucose and TAG concentrations.

Aim 1a: To determine if alterations in pancreatic (insulin, c-peptide, and glucagon) and gut (glucose-dependent insulinotropic polypeptide and glucagon-like peptide-one) hormone concentrations, whole body substrate oxidation, gastric emptying, free fatty acids, and beta-cell function are related to RE induced changes in postprandial glucose and TAG concentrations.

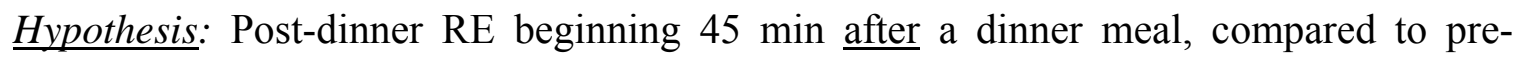
dinner RE ending $\sim 30$ min prior to a dinner meal, will result in lower $4 \mathrm{~h}$ postprandial glucose, TAG, insulin, c-peptide, GIP, free fatty acids, and gastric emptying responses but a greater GLP-1 response and enhanced beta-cell function in individuals with T2D. The originally proposed $4 \mathrm{~h}$ postprandial responses are illustrated in Table 2 .

Specific Aim 2: To determine if post-dinner RE is more effective compared to pre-dinner $\mathrm{RE}$ at lowering nocturnal (12 - 6 a.m.) blood glucose concentrations, fasting morning blood glucose concentrations, and postprandial blood glucose concentrations to a standardized breakfast meal the next morning.

Hypothesis: Post-meal RE will be more effective compared to pre-dinner RE at improving nocturnal blood glucose concentrations and fasting and postprandial glucose concentrations the next morning because post-meal RE will be performed closer to when these measurements are taken. 
Specific Aim 3: To determine how RE timing impacts subjective well-being (pleasure).

Hypothesis: Subjective well-being will be greater when RE is performed compared to no exercise, independent of its timing.

Table 2. Hypothesized $4 \mathrm{~h}$ postprandial responses during each trial.

\begin{tabular}{|c|c|c|c|}
\hline Organ & No Exercise & Pre-meal RE & Post-meal RE \\
\hline Stomach & $\downarrow$ Gastric Emptying & $\downarrow$ Gastric Emptying & $\downarrow \downarrow$ Gastric Emptying \\
\hline & $\begin{array}{l}\uparrow \uparrow \text { Glucose Output } \\
\uparrow \text { GLP-1 } \\
\uparrow \uparrow \text { GIP }\end{array}$ & $\begin{array}{l}\uparrow \uparrow \text { Glucose Output } \\
\uparrow \text { GLP-1 } \\
\uparrow \uparrow \text { GIP }\end{array}$ & $\begin{array}{l}\uparrow \text { Glucose Output } \\
\uparrow \uparrow \text { GLP-1 } \\
\uparrow \text { GIP }\end{array}$ \\
\hline Liver & $\begin{array}{l}\uparrow \text { Glucose Uptake } \\
\downarrow \text { Glucose Output }\end{array}$ & $\begin{array}{l}\uparrow \text { Glucose Uptake } \\
\downarrow \text { Glucose Output }\end{array}$ & $\begin{array}{l}\uparrow \text { Glucose Uptake } \\
\downarrow \text { Glucose Output }\end{array}$ \\
\hline & $\uparrow$ Glucose Uptake & $\uparrow$ Glucose Uptake & $\uparrow \uparrow$ Glucose Uptake \\
\hline Pancreas & $\begin{array}{l}\uparrow \uparrow \text { Insulin } \\
\uparrow \uparrow \text { c-peptide } \\
\leftrightarrow \text { Glucagon } \\
\downarrow \downarrow \text { Beta Cell Function }\end{array}$ & $\begin{array}{l}\uparrow \text { Insulin } \\
\uparrow \text { c-peptide } \\
\stackrel{\leftrightarrow}{\leftrightarrow} \text { Glucagon } \\
\uparrow \text { Beta Cell Function }\end{array}$ & $\begin{array}{l}\uparrow \text { Insulin } \\
\uparrow \text { c-peptide } \\
\stackrel{\leftrightarrow}{\leftrightarrow} \text { Glucagon } \\
\uparrow \uparrow \text { Beta Cell Function }\end{array}$ \\
\hline Brain & $\downarrow$ Well-being (Pleasure) & $\uparrow \uparrow$ Well-being (Pleasure) & $\uparrow \uparrow$ Well-being (Pleasure) \\
\hline
\end{tabular}

\section{Significance and Innovation}

For the first time, this study examined if post-dinner RE is superior compared to pre-dinner RE at improving postprandial risk factors in individuals with T2D both in a lab setting and under free-living conditions outside of the lab. These findings have direct translational application for exercise prescription for individuals with T2D, as knowledge of how different RE times relative to a meal impacts risk factors and insulin sensitivity will allow more effective exercise prescriptions to be made that will maximize the metabolic benefits of RE. 


\section{CHAPTER 2 - EXPERIMENTAL DESIGN AND METHODS}

\section{Participants}

The University of Missouri Health Science Institutional Review Board approved this study protocol and all participants provided written informed consent. All participants included in this study were obese (defined as body mass index $>30 \mathrm{~kg} / \mathrm{m}^{2}$ and body fat $>22 \%$, assessed via the BOD POD $\left.{ }^{\circledR}\right)$, physician diagnosed with type 2 diabetes, receiving standard medical care, non-smokers, not using insulin, had no history of surgery for weight loss, refrained from performing exercise or going on any special diets while participating, and were weight stable while participating in this study. The participants were instructed to take their medications at the usual dose, frequency, and time and not to change this routine while participating in this study.

\section{General Study Design}

Prior to beginning the study, all participants performed baseline testing which included measuring resting energy expenditure and free-living physical activity energy expenditure, strength testing, and two familiarization sessions (described in detail later). Following this, each participant performed three different, $3 \mathrm{~d}$ trials (counterbalanced design, participants serve as their own controls) in a random order.

The study design is illustrated in Figure 2 (page 18).

Specific Aim 1: To determine if post-dinner RE is more effective compared to pre-dinner $\mathrm{RE}$ at improving postprandial glucose and TAG concentrations.

Aim 1a: To determine if alterations in pancreatic (insulin, c-peptide, and glucagon) and gut (glucose-dependent insulinotropic polypeptide and glucagon-like peptide-one) hormone concentrations, whole body substrate oxidation, gastric emptying, free fatty 
acids, and beta-cell function are related to RE induced changes in postprandial glucose and TAG concentrations.

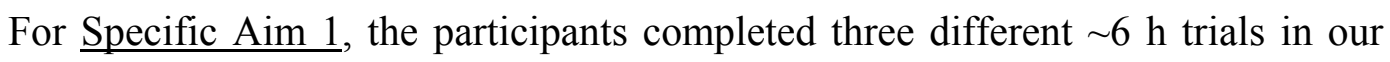
Exercise Physiology Lab. Each trial started between 3 - 5:30 p.m. (depending on the participants schedule), and upon arrival for each trial a venous catheter was inserted into the forearm of the participant. Blood samples were taken every 5 or 10 min during the first $4 \mathrm{~h}$ while in the lab, and then every 30 min during the last $2 \mathrm{~h}$ in the lab until the end of testing ( $\sim 9-11: 30$ p.m.). Sometime between $5-7$ p.m. (depending on arrival) the participants began eating a standardized dinner meal and were given 20 min to eat the meal. During the no RE trial (NoRE), the participants remained sedentary during testing. During the pre-dinner $\mathrm{RE}$ trial $(\mathrm{RE} \rightarrow \mathrm{M}), \sim 45$ min of $\mathrm{RE}$ was performed prior to dinner (ending $\sim 20-30$ min prior to dinner). During the post-dinner $R E$ trial $(\mathrm{M} \rightarrow \mathrm{RE}), \sim 45 \mathrm{~min}$ of $R E$ was performed beginning $45 \mathrm{~min}$ after finishing the meal. During the $\mathrm{M} \rightarrow \mathrm{RE}$ trial, the RE session began 45 min after dinner because we wanted the participants to finish the RE session prior to the postprandial glucose peak (which in our lab in well controlled $\mathrm{T} 2 \mathrm{D}$ is $\sim 1 \mathrm{~h}$ and 15 min after ingestion of a mixed meal) and prior to the contents of the meal being emptied from the stomach. Thus, the RE session would attenuate the blood glucose response to the meal to a greater extent than waiting longer after the meal. Acetaminophen was ingested with the dinner meal, and was used to indirectly assess gastric emptying. In addition to blood sampling, indirect calorimetry was used to measure energy expenditure and whole body substrate oxidation and subjective well-being measurements were taken after each blood draw for $\sim 6 \mathrm{~h}$ while the participants were in the lab (more specific details of these measurements are described later). After the last 
blood sample was taken the participants were released from the lab and allowed to resume normal daily activities and sleep in their own home.

Specific Aim 2: To determine if post-dinner RE is more effective compared to pre-dinner RE at lowering nocturnal (12 - 6 a.m.) blood glucose concentrations, fasting morning blood glucose concentrations, and postprandial blood glucose concentrations to a standardized breakfast meal the next morning.

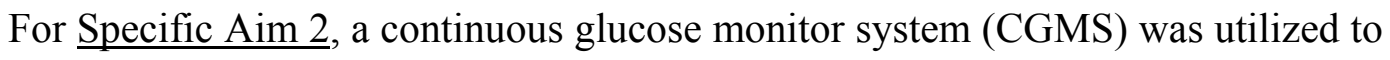
assess glucose concentrations under free-living conditions during the overnight period and to a standardized breakfast meal the morning after testing in the lab. During the evening of day one, the participants reported to the lab and the CGMS was inserted and the participants were trained on how to take and record a finger stick blood glucose measure. Following training, the participants were given their prepackaged study meals and instructed on when to eat the meals. On day two, the participants ate the prepackaged meals at the scheduled times on their own in the morning and afternoon. The evening meal was eaten in the lab while testing for Specific Aim 1 was occurring. Prior to the breakfast and lunch meal on day 2, the participants took and recorded a finger stick blood glucose measure, which was used to calibrate the CGMS. On day three of all trials, the participants reported to the lab in the morning after an overnight fast and a fasting blood sample was taken, and they were then given a standardized breakfast meal. The CGMS was used to measure the $4 \mathrm{~h}$ postprandial blood glucose response after breakfast. 
Specific Aim 3: To determine how resistance exercise timing impacts subjective wellbeing (pleasure).

For Specific Aim 3, a visual analog scale was used to assess the subjective wellbeing of each subject while testing in the lab was occurring. After every blood draw (every 5-10 min during the first $4 \mathrm{~h}$ of testing and every 30 min during the final $2 \mathrm{~h}$ of testing), the participants rated their subjective well-being.

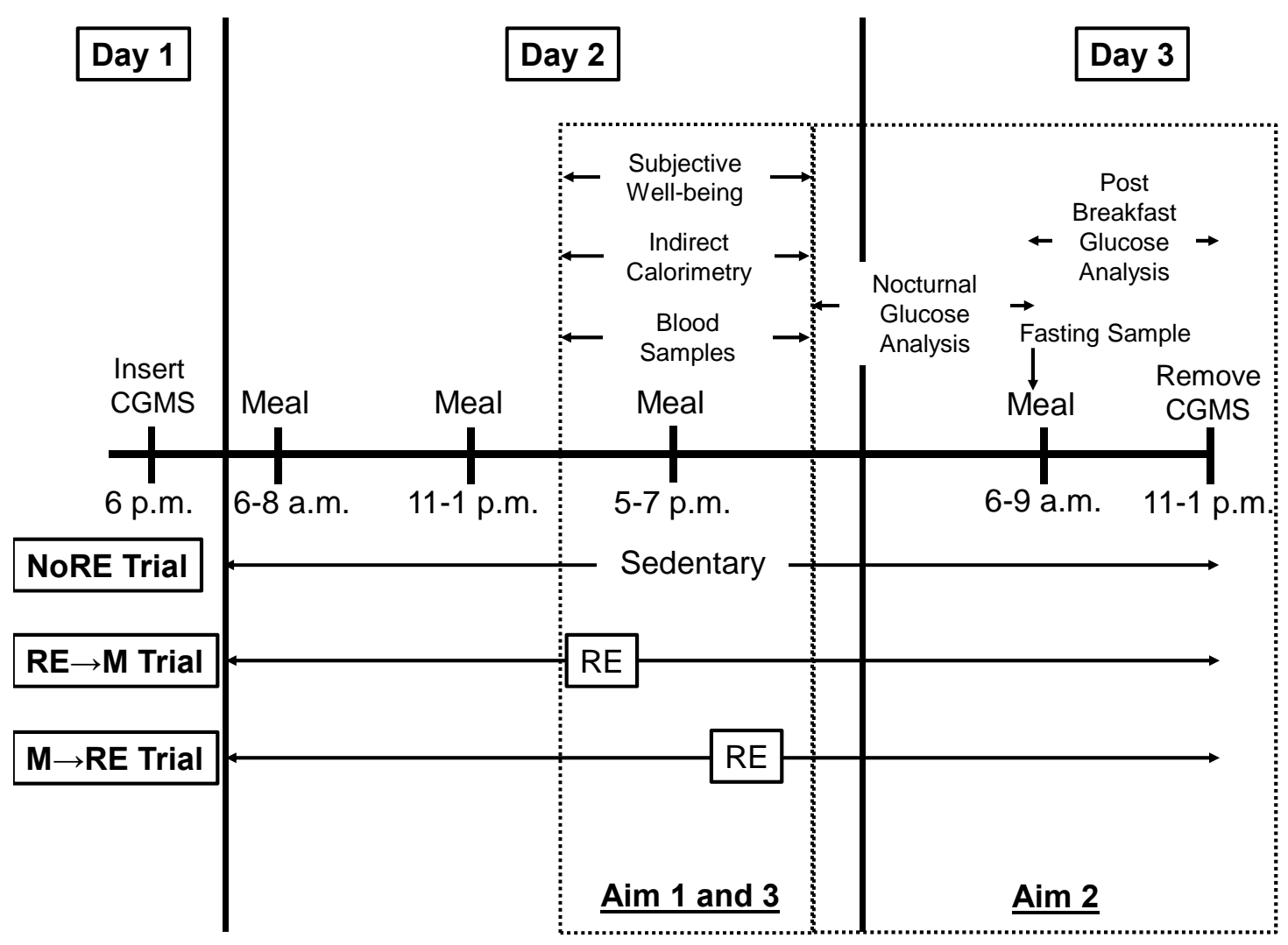

Figure 2. Study Design for Specific Aim 1, 2, and 3. 


\section{Detailed Methods}

Medications: During each trial the participants were instructed not to alter their medication use and to continue to take their prescription medication at the same dosage, time, and frequency that they have been taking them.

Diet: During day two and the morning of day three of each experimental trial, the participants were provided with a standardized diet. Prior to beginning the study, total daily energy expenditure was estimated for each participant. This was accomplished by measuring resting energy expenditure (REE) using indirect calorimetry (Parvomedics) and free-living physical activity energy expenditure with a BodyMedia ${ }^{\circledR}$ armband (BodyMedia, Inc, Pittsburgh, PA). The sum of these two measurements was used as an estimate of total daily energy requirements. For all the meals, the percent of energy from each macronutrient was $50 \%$ carbohydrate, $35 \%$ fat, and $15 \%$ protein. The breakfast meal was 585 Calories and consisted of an English muffin, cheddar cheese, one large egg, ham, hash brown, ketchup, and apple or orange juice. The lunch meal was 583 Calories and consisted of white bread, ham, mayonnaise, cheddar cheese, a granola bar, and apple or orange juice. The dinner meal was spaghetti noodles, spaghetti sauce with beef added, garlic bread, a lemon lime flavored soda, and $1.5 \mathrm{~g}$ of acetaminophen (to assess gastric emptying). The Caloric content of the dinner meal was calculated by subtracting 1,186 Calories (from breakfast and lunch meals) from the estimated total daily energy expenditure for that participant. On the morning of day 3 , the participants were given the same standardized breakfast that they consumed during day 2 .

Gastric Emptying: During day two, the participants ingested $1.5 \mathrm{~g}$ of acetaminophen with the dinner meal in the lab. The area under the curve (AUC) provides an estimate of the 
gastric emptying rate [70]. Prior research has shown that acetaminophen does not alter the glycemic response to a test meal in healthy or diabetic individuals [71] and pilot data in our lab showed that acetaminophen did not interfere with YSI or CGMS glucose measurements (unpublished data).

Indirect Calorimetry and Heart Rate: A ParvoMedics TrueOne ${ }^{\circledR} 2400$ metabolic measurement system (Sandy Lake, UT) was used to measure REE and energy expenditure and whole body substrate oxidation during exercise and throughout each trial according to manufacturer specifications. The REE measurement was conducted in the morning after an overnight fast. Prior to the REE measurement, the participants sat quietly for $\sim 10-15$ min prior to the REE measurement. During the REE, participants lied supine in a recliner for 30 min while all expired air was collected and analyzed for $\mathrm{O}_{2}$ and $\mathrm{CO}_{2}$ content. The $\mathrm{O}_{2}$ and $\mathrm{CO}_{2}$ values were converted to a $\mathrm{REE}$ estimate using the ParvoMedics software (Weir Equation).

During testing for each trial, indirect calorimetry was used to measure energy expenditure, $\mathrm{VO}_{2}$, and whole body substrate oxidation at three different time points including: 1 ) for a duration of $\sim 45 \mathrm{~min}$ prior to dinner (starting $\sim 115$ min prior to dinner and during exercise during the $\mathrm{RE} \rightarrow \mathrm{M}$ trial), 2) for a duration of $\sim 45$ min after dinner (starting $\sim 45$ min after dinner and during exercise during the $M \rightarrow R E$ trial), and 3) for a duration of 15-30 min starting $\sim 3.5 \mathrm{~h}$ after dinner. Heart rate was measured during exercise only using a Polar heart rate monitor.

Subjective Perceived Exertion and Well-being: Ratings of perceived exertion (RPE) were measured at the end of every RE set using Borg's 6-20 scale to determine how hard the participants perceived each set to be. Well-being was assessed using a computerized 0- 
$100 \mathrm{~cm}$ visual analog scale after every blood draw. The question was worded "How strong is your overall feeling of well-being (pleasure)" and the participants marked a single horizontal line through the vertical line on the scale in-between the anchors "not at all" and "extremely".

Blood Glucose Monitoring: A CGMS (iPro CGMS; Medtronic, Minneapolis, MN, USA) was used to assess free-living glucose responses during the testing period. The CGMS was inserted just beneath the skin in the periumbilical region of the abdomen. The participants were instructed to take and record a finger stick blood glucose (Accu-Chek Compact Plus; Roche Diagnostics, Indianapolis, IN), and to take one finger stick blood glucose prior to each meal during the testing period. The fingerstick blood glucose readings were used to calibrate the CGMS according to manufacturer specifications. Data from the CGMS was processed using Solutions Software for CGMS iPro (Version 2.0A). Resistance Exercise: For baseline testing, each participant was familiarized with RE so that they were not naïve to RE before testing. First, the participants completed familiarization session one to teach them how to properly perform each exercise. During familiarization one, the weight for the resistance exercises was light $(\sim 10-40 \%$ of bodyweight) and the participants performed 1-2 sets of 10 repetitions with a 1 min rest period between each set of the following exercises (performed in this order): Leg press, seated calf raises, seated chest flies, seated back flies, back extensions, shoulder raises, leg curls, and abdominal crunches. Next, after familiarization session one, strength testing ensued and the 10 repetition maximum (10-RM) for each exercise described previously (except abdominal crunches) was determined. During 10-RM testing, each exercise began with a warm-up set $(\sim 10-40 \%$ of bodyweight), and then for each 
subsequent set additional weight was added until the 10-RM for the exercise was determined ( $\sim 2$ min of rest was allotted between each attempt). Lastly, after the strength testing session and after at least a $3 \mathrm{~d}$ recovery period, the participants performed familiarization session two. For familiarization session two, the participants performed three sets (1-2 min rest between sets) of 10 repetitions using the 10-RM weight determined previously for the exercises described previously. The first set for each exercise was a warm-up set and the weight used was $50 \%$ of the participants $10-\mathrm{RM}$. After the warm-up set, the weight for the next two sets was the participants previously determined 10-RM. After familiarization session one, strength testing, and familiarization session two were completed, the participants began performing the exercise trials. During the $\mathrm{RE} \rightarrow \mathrm{M}$ and $\mathrm{M} \rightarrow \mathrm{RE}$ trials, the acute bout of exercise during these trials was the same as familiarization session two.

Blood Collection and Analysis: Upon arrival for testing on day two, a venous catheter was inserted into the antecubital vein of the participant. During the first $4 \mathrm{~h}$ of testing a blood sample was taken every 5 or $10 \mathrm{~min}$, and during the last $\sim 2 \mathrm{~h}$ of testing a blood sample was taken every $30 \mathrm{~min}$. Blood samples at each time point $(\sim 1.5-8.5 \mathrm{~mL})$ were transferred immediately into chilled EDTA tubes pretreated with Aprotinin (Thermo Fisher Scientific, Inc, USA) only (for TAG samples), chilled EDTA tubes pretreated with dipeptidyl peptidase-4 inhibitor (Millipore, USA), Pefabloc ${ }^{\circledR}$ SC (DSM Nutritional Products AG, Switzerland), and Aprotinin (Millipore, USA, for hormone, glucose, and FFA), or EDTA tubes not treated with any protease inhibitors (for acetaminophen assay). Within 30 min from collection, the blood was separated by centrifugation at 3,000 RPM for $10 \mathrm{~min}$ at $4^{\circ} \mathrm{C}$ and frozen at $-80^{\circ} \mathrm{C}$ until analysis. 
Separation of Lipoprotein Species: Separation of plasma chylomicron $\left(\mathrm{S}_{\mathrm{f}}>400\right)$, VLDL1 ( $\mathrm{S}_{\mathrm{f}}$ 60-400), and VLDL-2 ( $\mathrm{S}_{\mathrm{f}}$ 20-60) was performed using density gradient ultracentrifugation, essentially as described by Karpe et al. [72], but with minor modification as we used a different rotor. Using fresh plasma, $0.1 \mathrm{~g}$ of $\mathrm{KBr}$ was mixed into $1 \mathrm{~mL}$ of plasma to bring the density up to $1.1 \mathrm{~g} / \mathrm{L}$ in a polyallomer ultracentrifuge tube (14 x $89 \mathrm{~mm}$. tube, Beckman Coulter, Inc, USA). Next, $3 \mathrm{ml}$ of $\mathrm{KBr}$ salt solution of density $1.1 \mathrm{~g} / \mathrm{l}$ was carefully layered on top of the plasma. $\mathrm{Next}, 4 \mathrm{ml}$ of $\mathrm{NaCl}$ solution of density $1.065 \mathrm{~g} / 1$ was layered on top of the $1.1 \mathrm{~g} / 1$ solution, followed by $3 \mathrm{ml}$ of $\mathrm{KBr}$ solution of density $1.020 \mathrm{~g} / \mathrm{l}$ being layered on top of the $1.065 \mathrm{~g} / \mathrm{l}$ solution. Lastly, $1 \mathrm{ml}$ of $\mathrm{NaCl}$ solution of density $1.006 \mathrm{~g} / 1$ was layered on top of the $1.020 \mathrm{~g} / 1$ solution. Ultracentrifugation was performed using a TH-641 swinging bucket rotor at 40,000 RPM and $10^{\circ} \mathrm{C}$. For the separation of each lipoprotein species, consecutive ultracentrifucations of $32 \mathrm{~min}$ (chylomicrons), $3 \mathrm{~h}$ and $28 \mathrm{~min}$ (VLDL-1), and $16 \mathrm{~h}$ (VLDL-2) were performed. After each run, the top $1 \mathrm{~mL}$ layer was carefully removed and frozen at $-80^{\circ} \mathrm{C}$ until analysis. Before the next run, the tube was refilled with $1 \mathrm{~mL}$ of $\mathrm{NaCl}$ solution of density $1.006 \mathrm{~g} / \mathrm{l}$.

Biochemical Analyses: Plasma TAG (Infinity ${ }^{\mathrm{TM}}$, Thermo Fisher Scientific Inc, USA), free fatty acids (Wako Chemicals USA, Inc. USA), and acetaminophen concentrations were determined using a colorimetric assay (Cambridge Life Science LTD, United Kingdom). Plasma hormone concentrations were determined from the same sample using a MILLIPLEX magnetic bead-based immunoassay (Millipore, USA). Hematocrit was measured after every blood draw and all samples were corrected for changes in plasma volume. Plasma volume variations $(\Delta \mathrm{VP})$ were calculated from hematocrit variations $(\mathrm{Ht}$, 
$\mathrm{Ht} 1=$ baseline $\mathrm{Ht}, \mathrm{Ht} 2=$ after baseline $\mathrm{Ht}$ ) using the following formula: $\% \Delta \mathrm{VP}=$ $100 *[(\mathrm{Ht} 1-\mathrm{Ht} 2) /(\mathrm{Ht} 2 *(100-\mathrm{Ht} 1))]$. Corrected plasma values were calculated using the following formula: Corrected Value $=($ Initial Value*100 $) /(100-\Delta V P)$.

Beta-cell function: Model based beta-cell function parameters were calculated using a mathematical model developed by Mari et al. [73-74] and the insulin secretion rates (ISR) used in the model were calculated by deconvoluting c-peptide concentrations [75]. Briefly, beta-cell glucose sensitivity is the slope of the dose response relation between insulin secretion, glucose concentration, and the potentiation factor. The potentiation factor ratio is the ratio between potentiation at the end of the dinner meal (220-240 min) and the initial value (0-20 min). Rate sensitivity represents the enhancement of insulin secretion proportional to the rate of change of plasma glucose.

Calculations: For specific aim 1 and 3, postprandial responses were quantified using the incremental area under the curve (iAUC), which was calculated using the trapezoidal method [76]. Gastric emptying was quantified using the iAUC. For specific aim 2, the data from the CGMS included 1) average nocturnal glucose concentration from 12-6 a.m., 2) time spent hyperglycemic (blood glucose $>180 \mathrm{mg} / \mathrm{dL}$ ), 3) time spent hypoglycemic (blood glucose $<70 \mathrm{mg} / \mathrm{dL}$ ), 4) the 4 h postprandial glucose iAUC to the breakfast meal on day 3 of each protocol, 5) the average morning blood glucose concentrations (6 a.m. to 12 p.m.), and 6) $24 \mathrm{~h}$ average blood glucose concentrations and $24 \mathrm{~h}$ glycemic variability from the start of lunch on day two thru the beginning of lunch on day three of each testing trial. Glycemic variability was assessed using the M-value [77], mean amplitude of glycaemic excursion (MAGE) [78], J-index [79], continuous overlapping net glycaemic action (CONGA-1, -2 , and -4) [80], and the liability index 
[81]. Insulin clearance was calculated as the molar ratio of insulin to c-peptide at each time point, as a percent $((1-[$ insulin/c-peptide pmol/L] $) * 100)$. The change in the glucose and TAG iAUC was calculated by subtracting the iAUC during the NoRE trial from the iAUC during either the $\mathrm{M} \rightarrow \mathrm{RE}$ or $\mathrm{RE} \rightarrow \mathrm{M}$ trial.

Statistical Analysis: Statistical analyses were performed using the IBM SPSS statistical software version 20 (IBM Corporation, Armonk, NY, USA). For specific aim 1 and 3, a repeated measures ANOVA with follow-up Bonferroni adjusted post hoc t-tests were used to compare the $4 \mathrm{~h}$ post-dinner iAUC responses for all variables. Significance was set at $\mathrm{P} \leq 0.05$. For Specific Aim 2, a repeated measures ANOVA with Bonferroni adjusted post hoc t-tests was used to compare 1) average nocturnal glucose concentration from 12-6 a.m., 2) time spent hyperglycemic (blood glucose $>180 \mathrm{mg} / \mathrm{dL}$ ), 3) time spent hypoglycemic (blood glucose $<70 \mathrm{mg} / \mathrm{dL}$ ), 4) the 4 h post-meal glucose iAUC to the breakfast meal on day 3 of each protocol, 5) the average glucose concentrations from 6 a.m. to 12 p.m. on day three of each trial and from the start of lunch on day two thru the start of lunch on day three, and 6) all of the glycemic variability indexes listed above. The values for the baseline characteristics are reported as means \pm standard deviation, while all other values are reported as means \pm standard error of the mean. 


\section{CHAPTER 3 - RESULTS}

\section{Participant Characteristics}

The participant characteristics are listed in Table 3. A total of 13 obese (BMI 30$\left.45 \mathrm{~kg} / \mathrm{m}^{2}\right)$ men and women (5 males) with type 2 diabetes completed this study. The participants were weight stable while enrolled in this study as body weight was not significantly different $(\mathrm{P}>0.05)$ between study days (NoRE body weight: $104.2 \pm 22.8$ $\mathrm{kg}$; RE $\rightarrow$ M body weight: $103.9 \pm 22.7 \mathrm{~kg} ; \mathrm{M} \rightarrow \mathrm{RE}$ body weight: $104.2 \pm 22.9 \mathrm{~kg}$ ).

Table 3. Participant Characteristics ( $\mathrm{N}=13,5$ Males)

\begin{tabular}{lc}
\hline Age $(\mathrm{yr})$ & $48.5 \pm 11.9$ \\
Height $(\mathrm{m})$ & $1.67 \pm 0.11$ \\
Weight $(\mathrm{kg})$ & $103.2 \pm 22.8$ \\
Body mass index $\left(\mathrm{kg} / \mathrm{m}^{2}\right)$ & $36.7 \pm 5.3$ \\
Body fat percent & $39.5 \pm 8.6$ \\
Fasting Glucose $(\mathrm{mg} / \mathrm{dL})$ & $148 \pm 41$ \\
Fasting Hemoglobin A1c (\% [mmol/mol]) & $7.2 \pm 1.1[55]$ \\
Diagnosed with Type 2 Diabetes (yr) & $3.7 \pm 3.9$ \\
Participants using diabetes medication, $\mathrm{n}$ & 12 \\
\hline
\end{tabular}

Values are means \pm standard deviation 


\section{Indirect Calorimetry, Heart Rate, and Rating of Perceived Exertion During Exercise}

The metabolic data and average rating of perceived exertion during each RE session are listed in Table 4. The average heart rate was 4 beats per minute higher $(\mathrm{P}<$ $0.05)$ during the $\mathrm{M} \rightarrow \mathrm{RE}$ trial $(110 \pm 4 \mathrm{bpm})$ compared to the $\mathrm{RE} \rightarrow \mathrm{M}$ trial $(106 \pm 4 \mathrm{bpm})$, while all other variables were similar between $\mathrm{RE}$ trials $(\mathrm{P}>0.05)$. In addition, $\mathrm{RE}$ significantly increased oxygen consumption, energy expenditure, and the respiratory exchange ratio compared to $\operatorname{NoRE}(\mathrm{P}<0.05)$.

Table 4. Metabolic data and rating of perceived exertion during resistance exercise session.

\begin{tabular}{lcc}
\hline & $\mathrm{RE} \rightarrow \mathrm{M}$ & NoRE-1 \\
\hline Time (min) & $46 \pm 1$ & $46 \pm 1$ \\
Oxygen consumption ( $\left.\mathrm{ml} \mathrm{kg}^{-1} \mathrm{~min}^{-1}\right)$ & $6.1 \pm 0.4$ & $2.9 \pm 0.1^{*}$ \\
Energy Expenditure (Gross Calories) & $140 \pm 14$ & $63 \pm 3^{*}$ \\
Respiratory Exchange Ratio & $1.00 \pm 0.01$ & $0.83 \pm 0.01^{*}$ \\
Heart Rate (bpm) & $106 \pm 4$ & \\
Average RPE (BORG 6-20 scale) & $12 \pm 1$ & \\
\hline & $\mathrm{M} \rightarrow \mathrm{RE}$ & NoRE-2 \\
\hline Time (min) & $47 \pm 1$ & $47 \pm 1$ \\
Oxygen consumption (ml kg-1 $\mathrm{min}^{-1}$ ) & $6.0 \pm 0.3$ & $2.9 \pm 0.1^{*}$ \\
Energy Expenditure (Gross Calories) & $141 \pm 14$ & $66 \pm 5^{*}$ \\
Respiratory Exchange Ratio & $1.00 \pm 0.01$ & $0.87 \pm 0.01^{* \dagger}$ \\
Heart Rate (bpm) & $110 \pm 4 * *$ & \\
Average RPE (BORG 6-20 scale) & $12 \pm 1$ & \\
\hline Values are means \pm standard err of the &
\end{tabular}

Values are means \pm standard error of the mean.

The data under NoRE-1 is during the same time frame (i.e. before dinner) as $\mathrm{RE} \rightarrow \mathrm{M}$ but during the NoRE trial, while the data under NoRE-2 is during the same time frame (i.e. after dinner) as $\mathrm{M} \rightarrow \mathrm{RE}$ but during the NoRE trial. $* \mathrm{P}<0.05$ compared to $\mathrm{RE} \rightarrow \mathrm{M}$ or $\mathrm{M} \rightarrow \mathrm{RE}$.

$* * \mathrm{P}<0.05$ compared to heart rate during the $\mathrm{RE} \rightarrow \mathrm{M}$ trial. $\dagger \mathrm{P}<0.05$ compared to NoRE-1.

For the variables time, oxygen consumption, energy expenditure, respiratory exchange ratio, and heart rate, only data for $\mathrm{N}=12$ is reported. For RPE, data for $\mathrm{N}=13$ is reported. 


\section{Blood Glucose Concentrations During Mixed Meal Testing}

The blood glucose time course (Figure 3A), pre-dinner iAUC (Figure 3B), and post-dinner iAUC (Figure 3C) are illustrated in Figure 3. Performing RE prior to dinner reduced blood glucose concentrations, as the $1.6 \mathrm{~h}$ blood glucose iAUC was significantly lower $(\mathrm{P}<0.05)$ during the $\mathrm{RE} \rightarrow \mathrm{M}$ trial compared to the NoRE and $\mathrm{M} \rightarrow \mathrm{RE}$ trials. The postprandial blood glucose iAUC was reduced by $33 \%$ and $18 \%$ during the $\mathrm{M} \rightarrow \mathrm{RE}$ trial and $\mathrm{RE} \rightarrow \mathrm{M}$ trial, respectively, compared to the NoRE trial (both $\mathrm{P}<0.05$ ). The $4 \mathrm{~h}$ blood glucose iAUC was not different between exercise trials $(\mathrm{P}>0.05)$.

\section{A. Glucose Time Course}

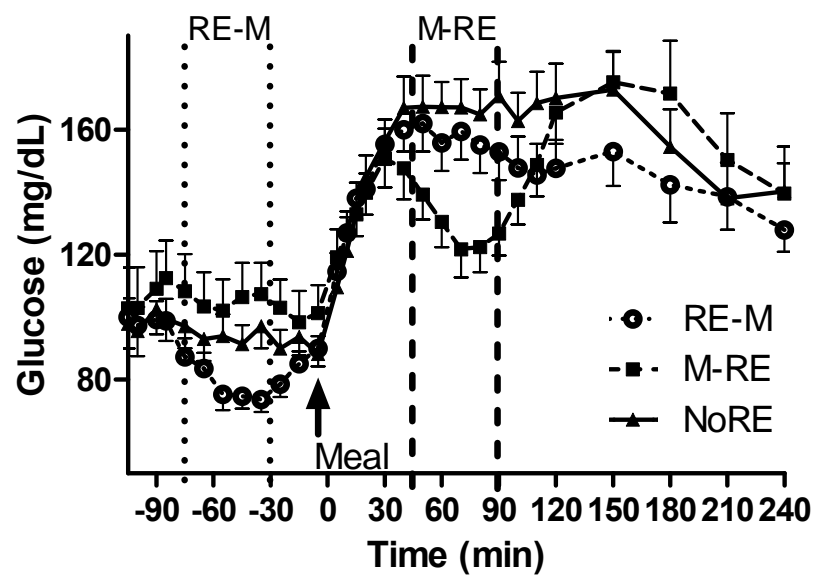

B. Pre-meal Glucose iAUC

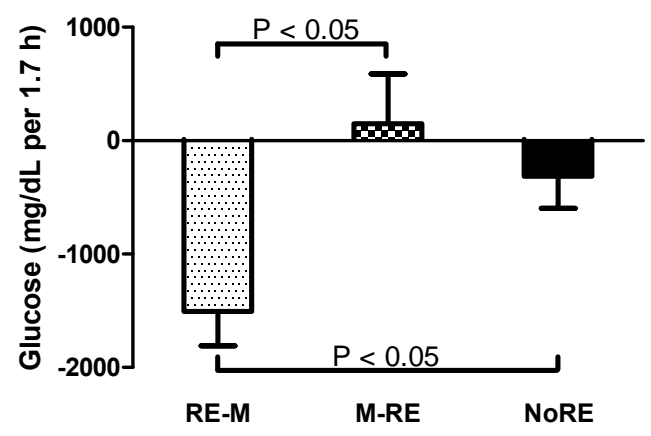

C. Post-meal Glucose iAUC

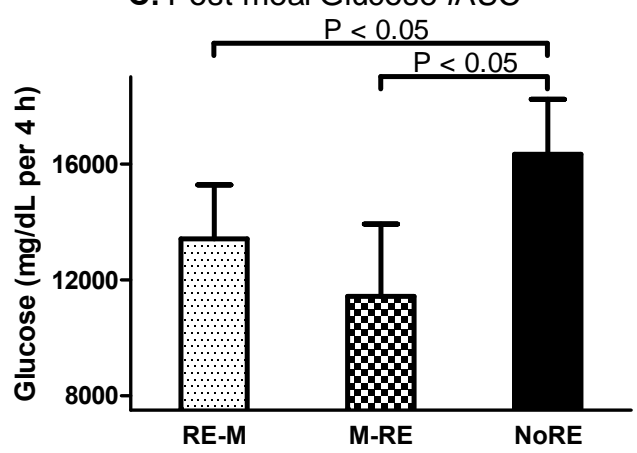

Figure 3. Blood Glucose Concentrations During Mixed Meal Testing. Values are means \pm standard error of the mean. 


\section{Individual Glucose Responses}

Figure 4 illustrates the change in the $4 \mathrm{~h}$ glucose iAUC during each RE trial for each individual person that participated in this study. Interestingly, 6 / 13 people had better improvements in glycemic control with post-meal RE, while 6/13 people had better improvements with pre-meal RE, and one person did not see an improvement with $\mathrm{RE}$ at either time. Thus, overall it appears that neither RE time is better compared to the other at lowering post-meal glucose concentrations. In three cases, it appears that RE timing played a role in exercise insensitivity. For example, subject 6 had a worsening of glycemic control with pre-meal RE, but post-meal RE improved glycemic control in this individual. Subject 10 and 11 had a worsening of glycemic control with post-meal RE, but pre-meal RE improved glycemic control in these individuals.

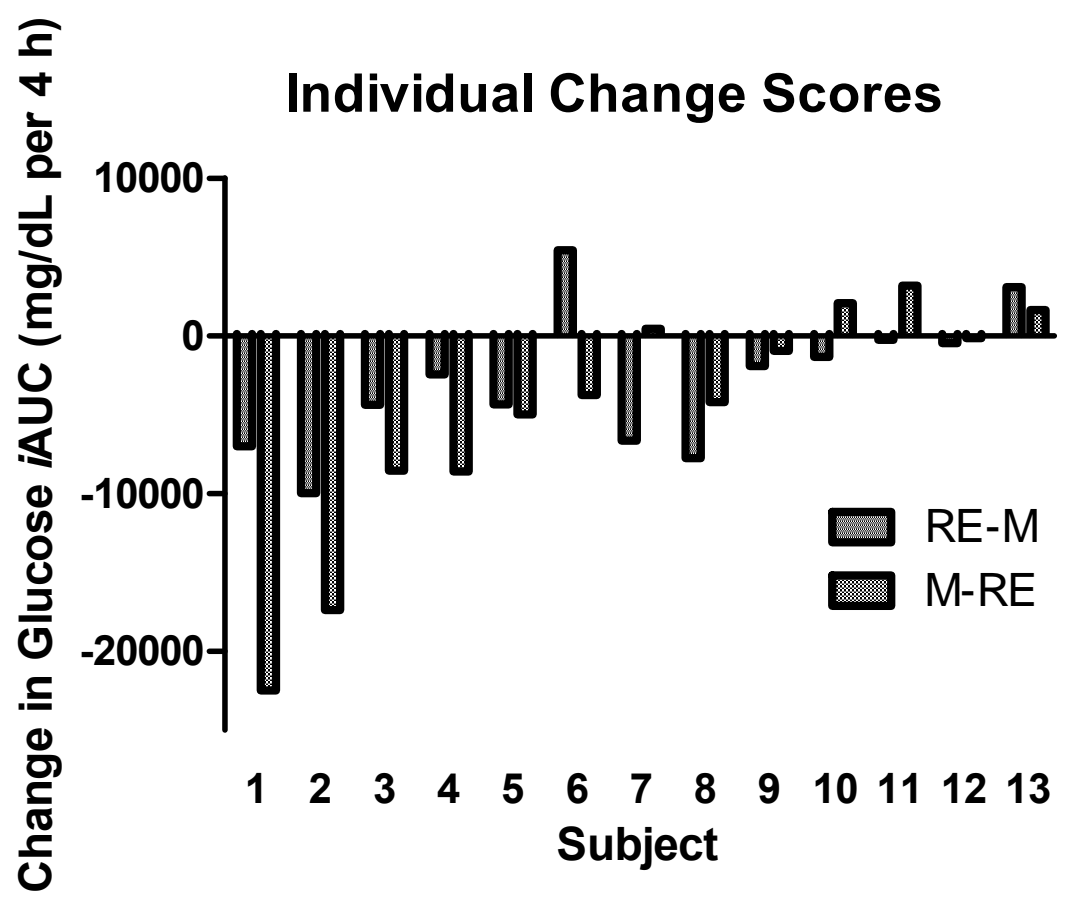

Figure 4. Change in glucose iAUC during each exercise trial in each subject. 


\section{Triacylglycerol Concentrations During Mixed Meal Testing}

The total TAG, chylomicron TAG, VLDL-1 TAG, and VLDL-2 TAG time course and iAUC during each trial are illustrated in Figure 5A and 5B, 6A and 6B, 7A and 7B, and $\mathbf{8 A}$ and $\mathbf{8 B}$, respectfully. During the $\mathrm{M} \rightarrow \mathrm{RE}$ trial, the postprandial total TAG iAUC and VLDL-1 TAG iAUC was reduced by $\sim 92 \%$ compared to the NoRE and RE $\rightarrow \mathrm{M}$ trials $(\mathrm{P}<0.05)$. The chylomicron TAG iAUC and VLDL-2 TAG iAUC was not different between trials $(\mathrm{P}>0.05)$.
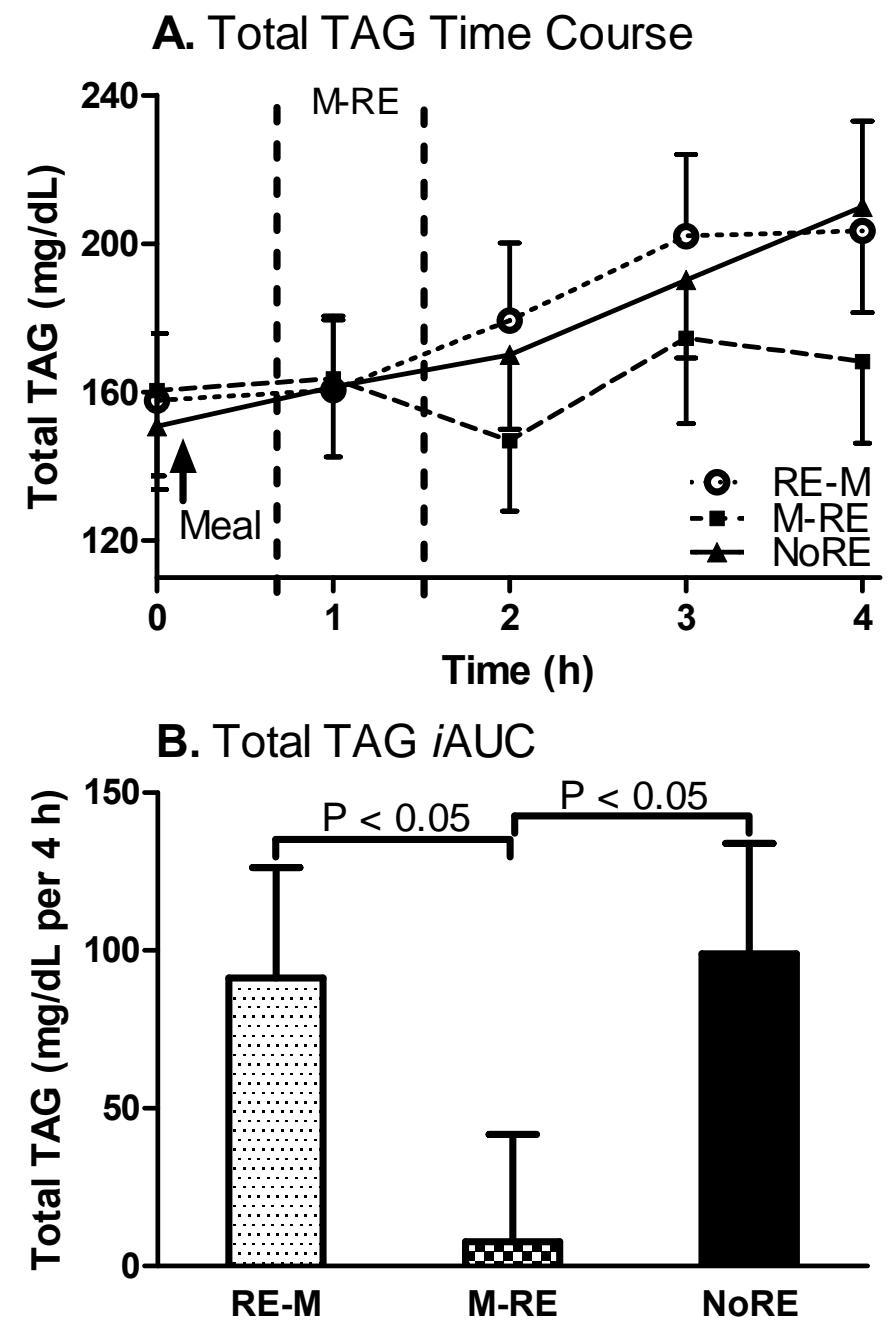

Figure 5. Total Triacylglycerol Concentrations During Mixed Meal Testing. Values are means \pm standard error of the mean. 


\section{A. Chylomicron TAG Time Course}
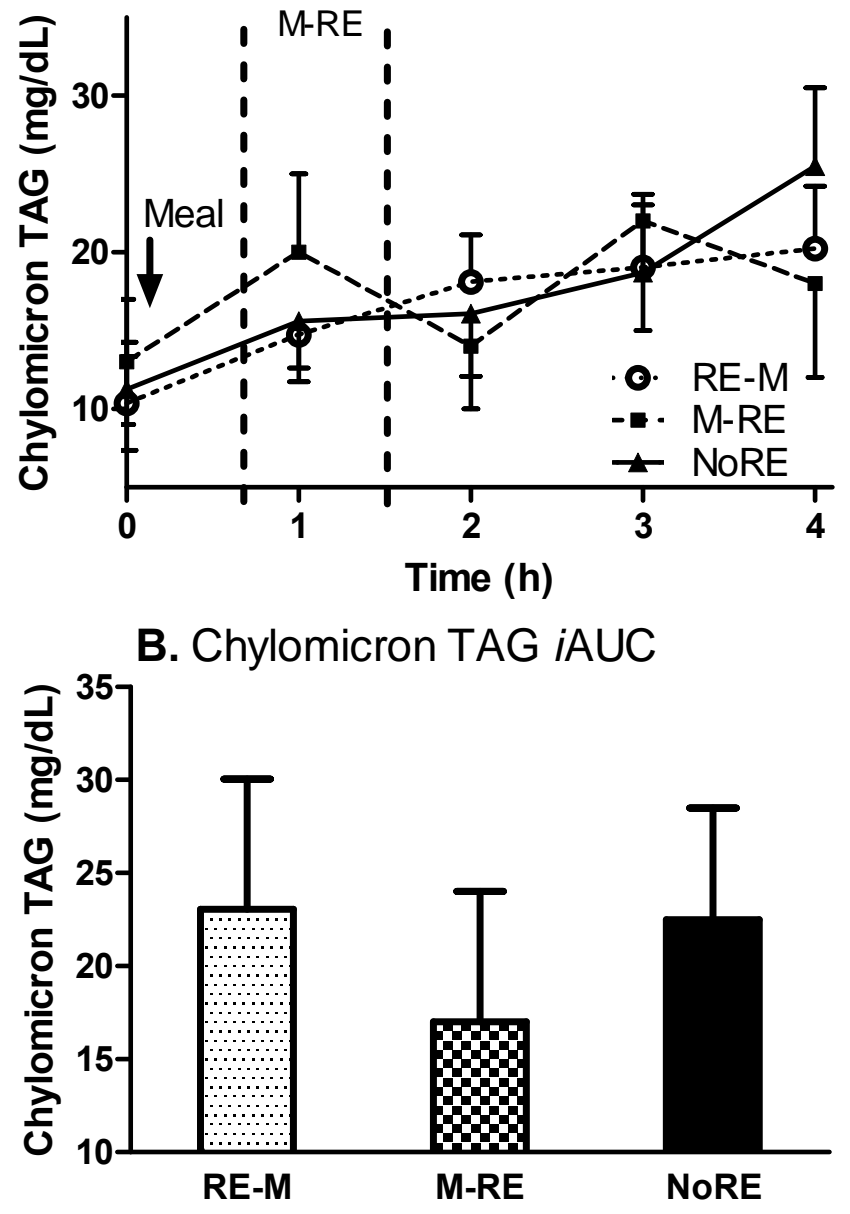

Figure 6. Chylomicron Triacylglycerol Concentrations During Mixed Meal Testing. Values are means \pm standard error of the mean. 
A. VLDL-1 TAG Time Course
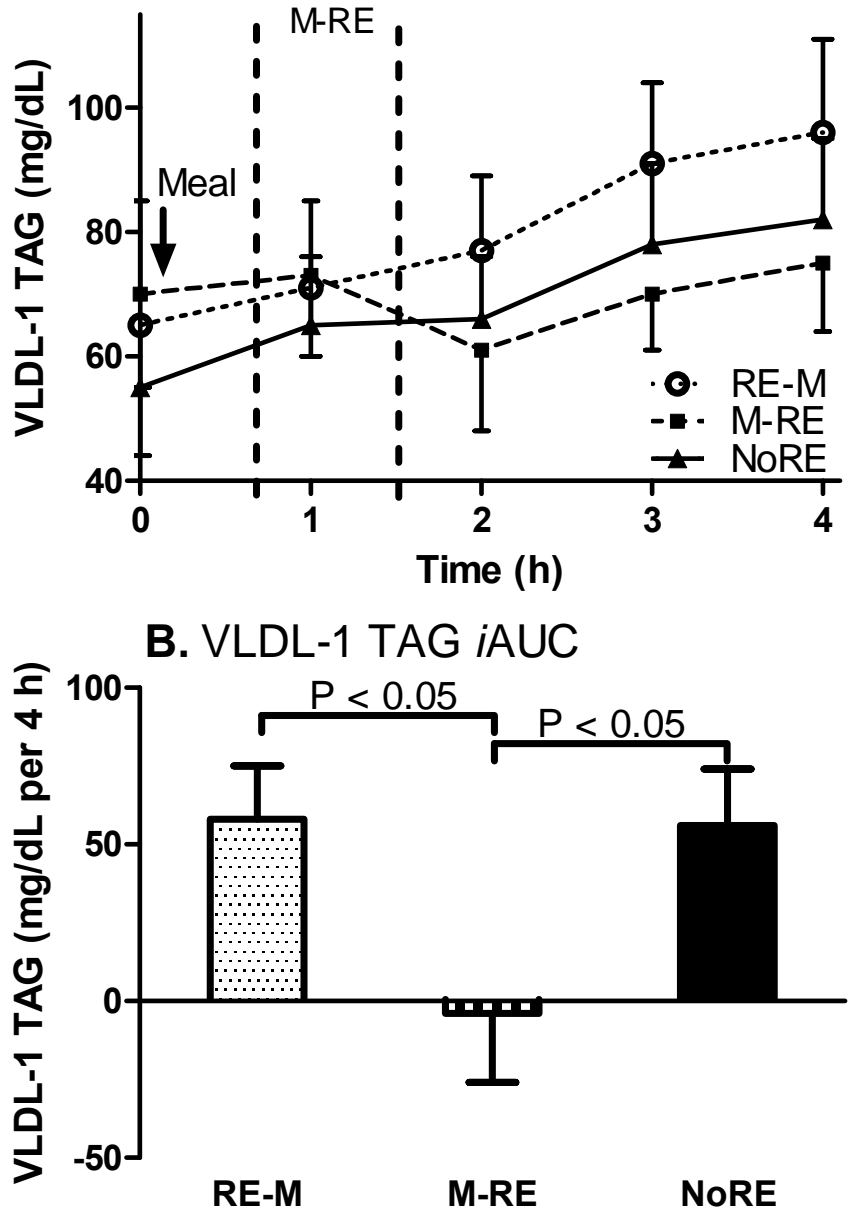

Figure 7. VLDL-1 Triacylglycerol Concentrations During Mixed Meal Testing. Values are means \pm standard error of the mean. 
A. VLDL-2 TAG Time Course
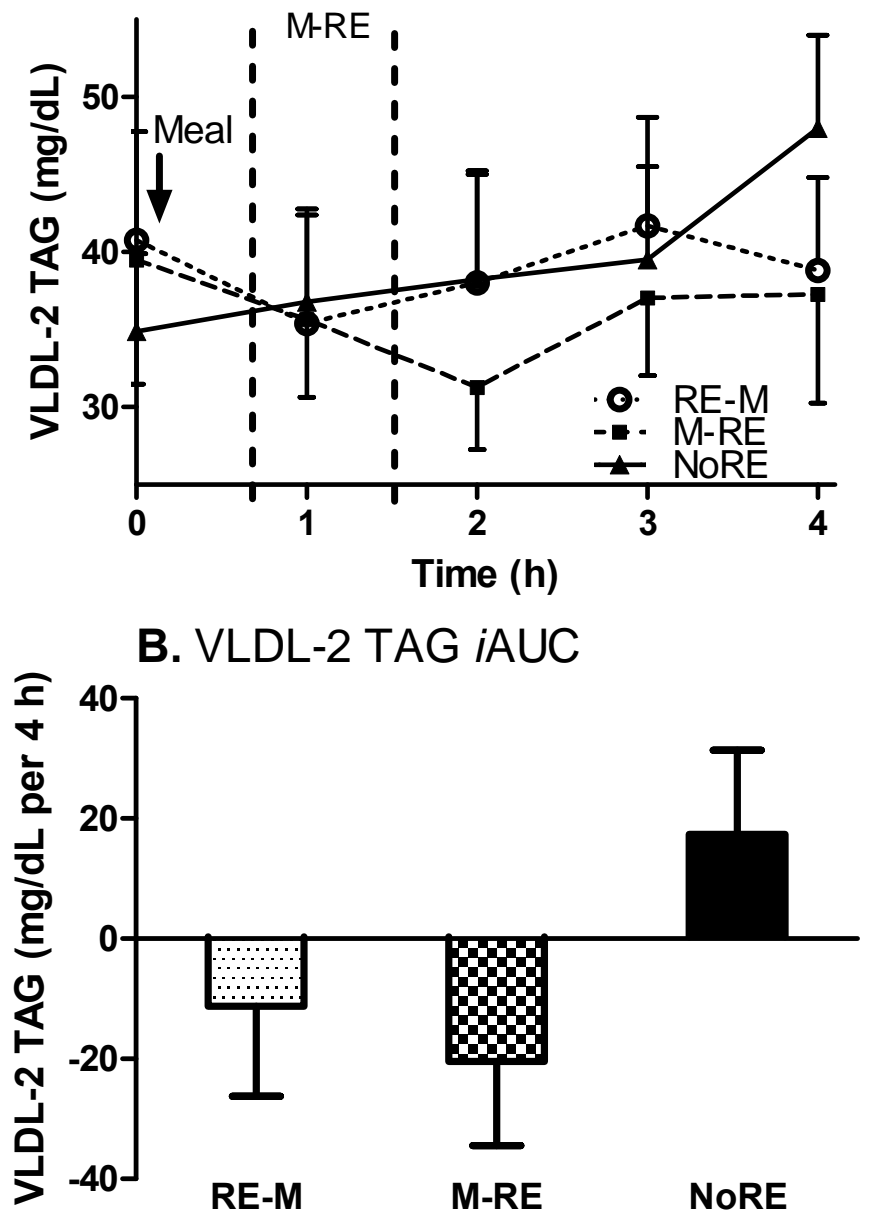

Figure 8. VLDL-2 Triacylglycerol Concentrations During Mixed Meal Testing. Values are means \pm standard error of the mean. 


\section{Individual Triacylglycerol Responses}

Figure 9 illustrates the individual TAG responses during each RE trial in each subject. In 7 / 13 people, post-meal RE was better at lowering postprandial TAG concentrations compared to pre-meal RE, while pre-meal RE was better (only slightly) in 3 / 13 people compared to post-meal RE. In three individuals, neither pre-meal nor postmeal RE improved postprandial TAG concentrations.

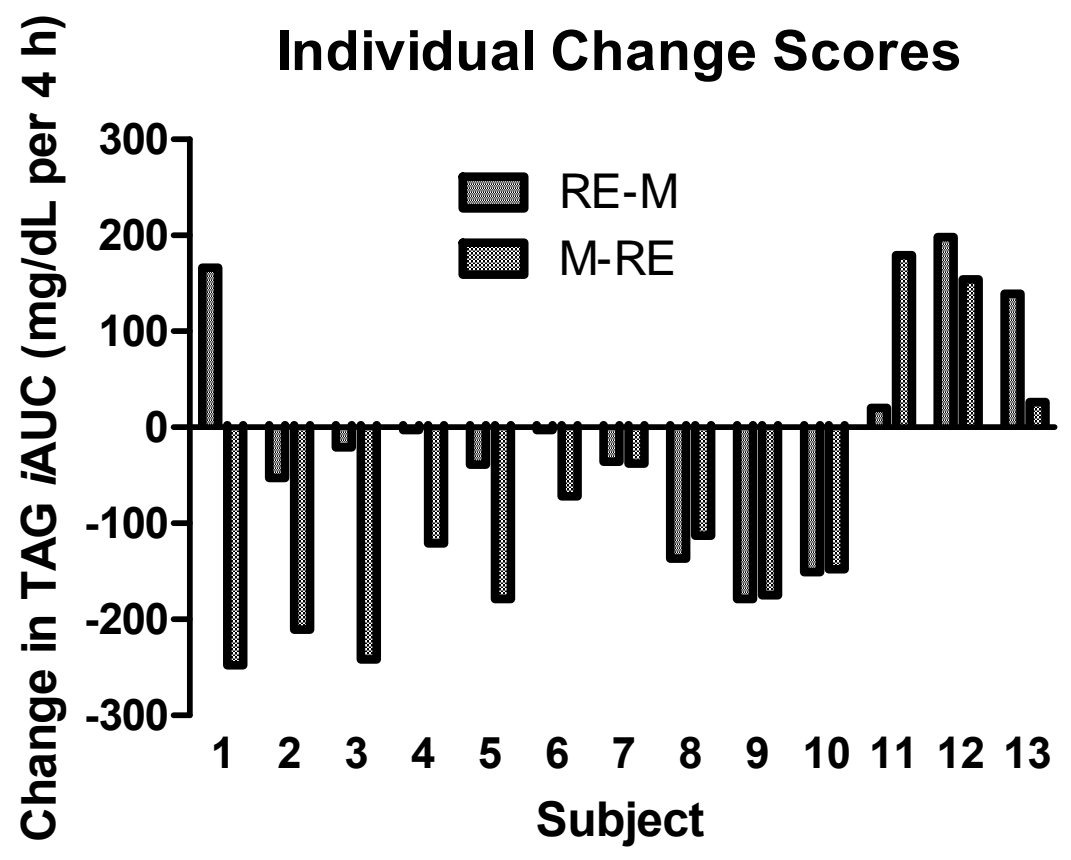

Figure 9. Change In Triacylglycerol iAUC During Each Exercise Trial In Each Subject. 


\section{Free Fatty Acid Concentrations During Mixed Meal Testing}

The plasma FFA time course (Figure 10A), pre-dinner FFA iAUC (Figure 10B), and post-dinner FFA iAUC (Figure 10C) during testing are illustrated in Figure 10. The rise in pre-meal FFA concentrations was significantly attenuated (lower pre-meal $1.6 \mathrm{~h}$ FFA $i$ AUC) during the $\mathrm{RE} \rightarrow \mathrm{M}$ trial compared to the NoRE and $\mathrm{M} \rightarrow \mathrm{RE}$ trials $(\mathrm{P}<0.05)$. Similarly, the drop in postprandial FFA concentrations was significantly less (higher [less negative] post-meal FFA iAUC) during the $\mathrm{RE} \rightarrow \mathrm{M}$ trial compared to the NoRE and $\mathrm{M} \rightarrow \mathrm{RE}$ trials $(\mathrm{P}<0.05)$.

A. Free Fatty Acid Time Course
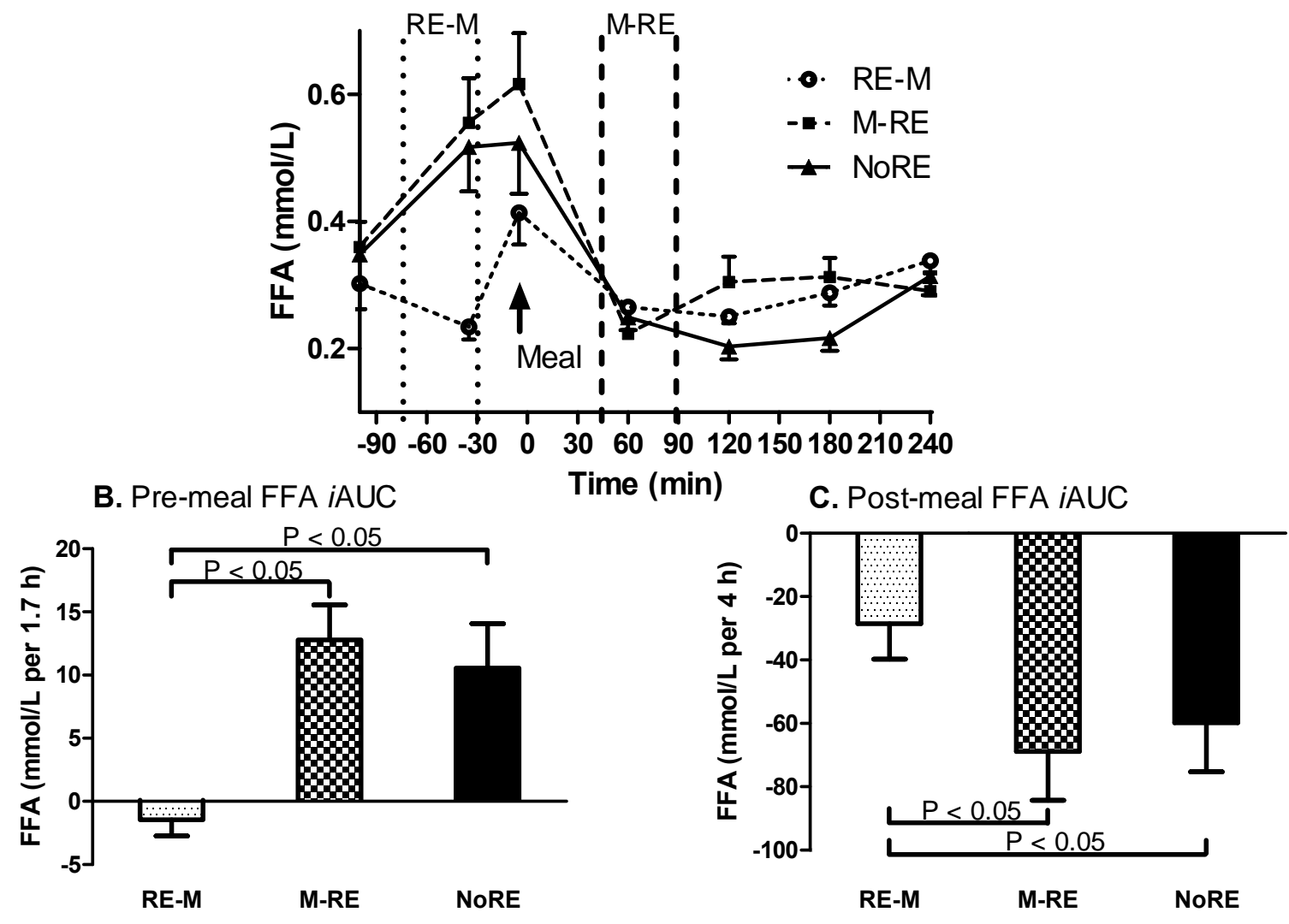

Time (min)

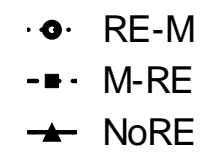

$\mp$ NoRE

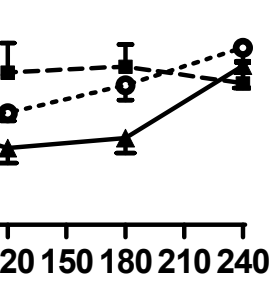

Figure 10. Free Fatty Acid Concentrations During Mixed Meal Testing. Values are means \pm standard error of the mean. 


\section{Insulin Concentrations During Mixed Meal Testing}

The insulin time course (Figure 11A), pre-dinner iAUC (Figure 11B), and postdinner iAUC (Figure 11C) are illustrated in Figure 11. The pre-dinner $1.6 \mathrm{~h}$ insulin iAUC was not different between trials $(\mathrm{P}>0.05)$. The postprandial $4 \mathrm{~h}$ insulin iAUC was $39 \%$ and $31 \%$ lower during the $\mathrm{M} \rightarrow \mathrm{RE}$ and $\mathrm{RE} \rightarrow \mathrm{M}$ trials, respectively, compared to the NoRE trial $(\mathrm{P}<0.05)$

A. Insulin Time Course

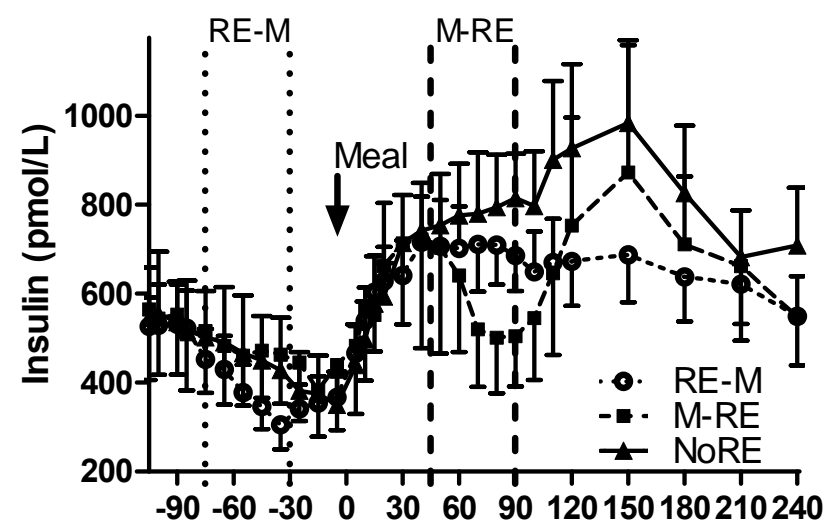

B. Pre-meal Insulin iAUC

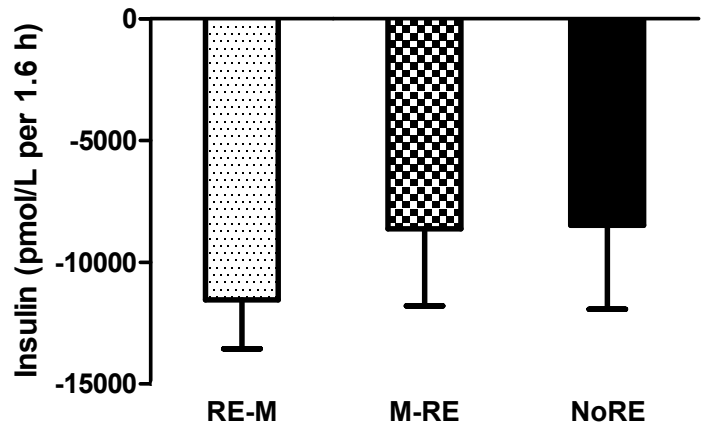

Time (min)

C. Post-meal Insulin iAUC

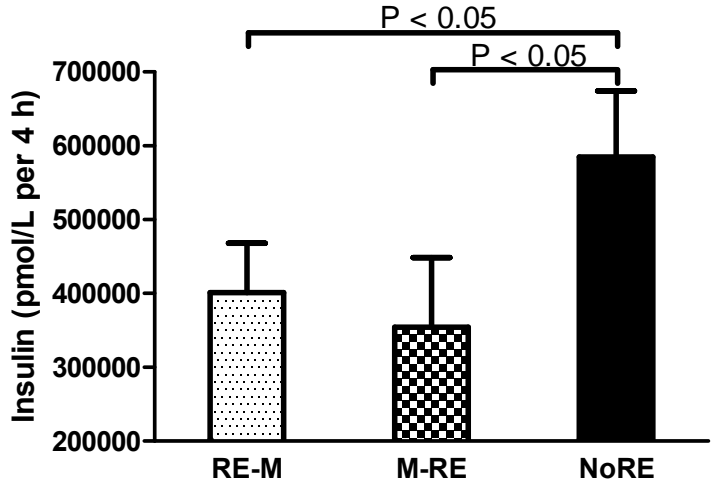

Figure 11. Insulin Concentrations During Mixed Meal Testing. Values are means \pm standard error of the mean. 


\section{C-peptide Concentrations During Mixed Meal Testing}

The c-peptide time course (Figure 12A), pre-dinner iAUC (Figure 12B), and post-dinner iAUC (Figure 12C) are illustrated in Figure 12. The pre-dinner $1.6 \mathrm{~h} \mathrm{c}$ peptide iAUC was significantly lower during the $\mathrm{RE} \rightarrow \mathrm{M}$ trial compared to the $\mathrm{M} \rightarrow \mathrm{RE}$ and NoRE trials $(\mathrm{P}<0.05)$, indicating $\mathrm{RE}$ decreased c-peptide concentrations prior to dinner. The postprandial $4 \mathrm{~h}$ c-peptide iAUC was $45 \%$ lower during the $\mathrm{M} \rightarrow \mathrm{RE}$ trial compared to the NoRE trial $(\mathrm{P}<0.05)$. Despite the postprandial $4 \mathrm{~h}$ c-peptide iAUC being $35 \%$ lower during the $\mathrm{M} \rightarrow \mathrm{RE}$ trial compared to the $\mathrm{RE} \rightarrow \mathrm{M}$, this difference was not significant $(\mathrm{P}>0.05)$.

A. C-peptide Time Course
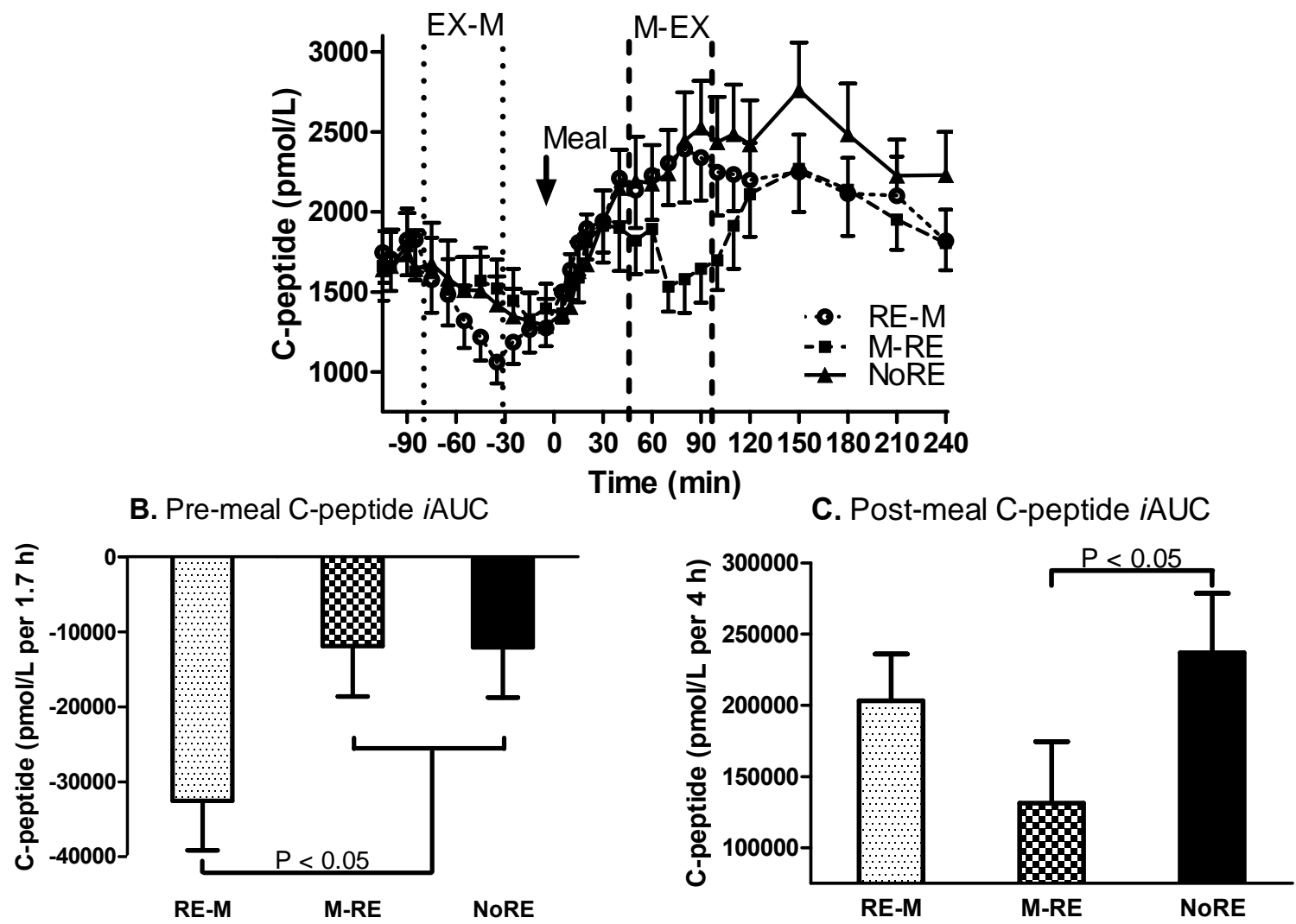

Figure 12. C-peptide Concentrations During Mixed Meal Testing. Values are means \pm standard error of the mean. 


\section{Insulin Kinetics During Mixed Meal Testing}

The insulin secretion time course (Figure 13A) and iAUC (Figure 13B) are illustrated in Figure 13. The postprandial $4 \mathrm{~h}$ ISR iAUC was $40 \%$ lower during the $\mathrm{M} \rightarrow \mathrm{RE}$ trial compared to the NoRE trial $(\mathrm{P}<0.05)$. There were no other significant differences in insulin secretion between trials $(\mathrm{P}>0.05)$.
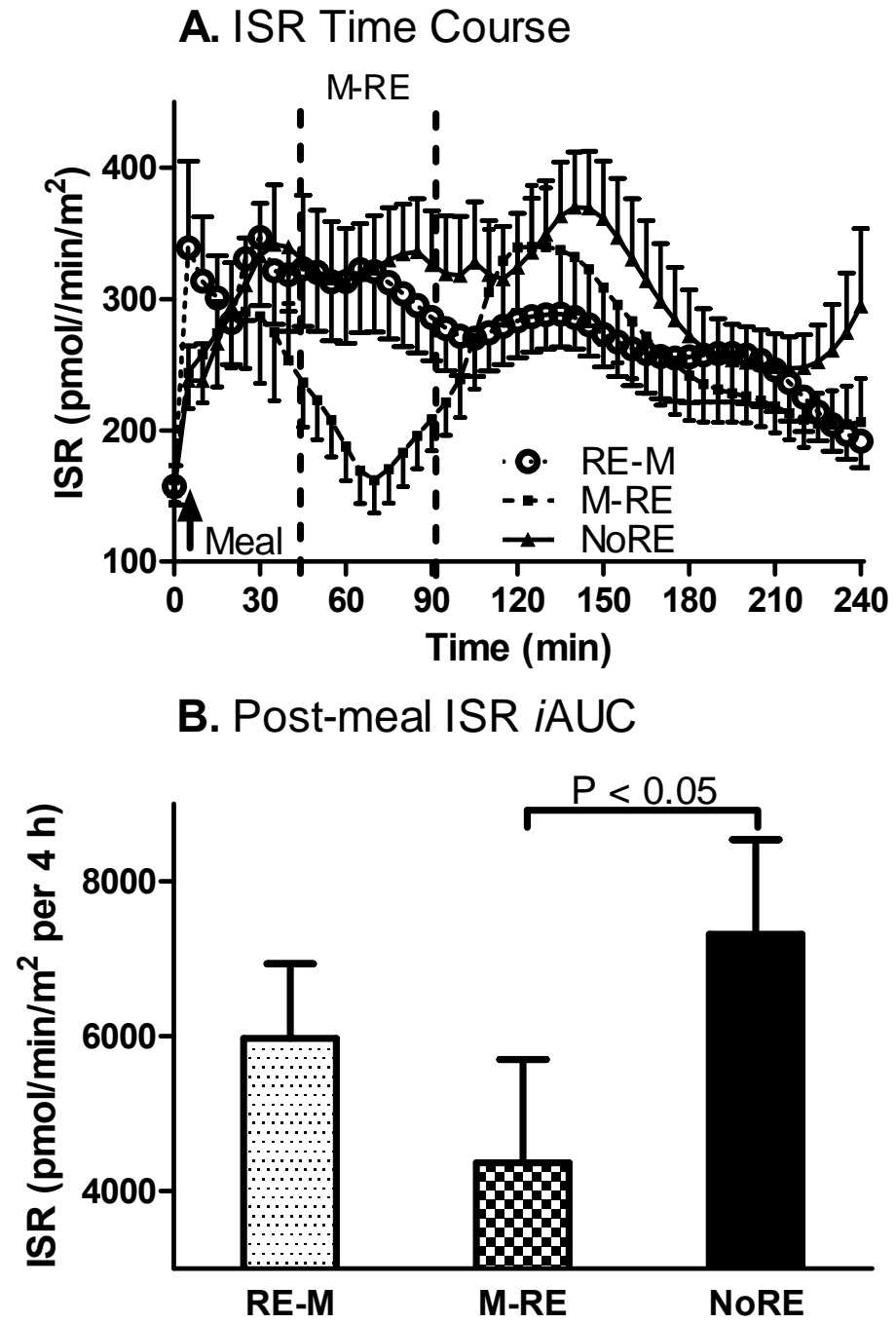

Figure 13. Insulin Secretion Rates During Mixed Meal Testing. Values are means \pm standard error of the mean. 
The insulin clearance time course (Figure 14A) and iAUC (Figure 14B) are illustrated in Figure 14. The postprandial $4 \mathrm{~h}$ insulin clearance iAUC was significantly different between each trial $(\mathrm{P}<0.05)$ and was highest in the $\mathrm{RE} \rightarrow \mathrm{M}$ trial, lowest in the NoRE trial, while insulin clearance during the $\mathrm{M} \rightarrow \mathrm{RE}$ trial was in between the other two trials.

A. Insulin Clearance Time Course

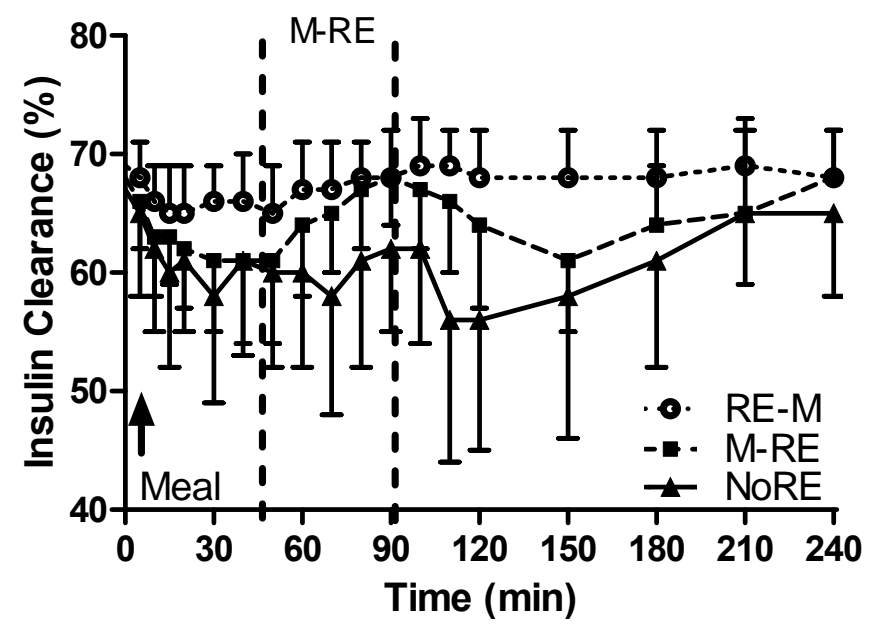

B. Post-meal Insulin Clearance iAUC

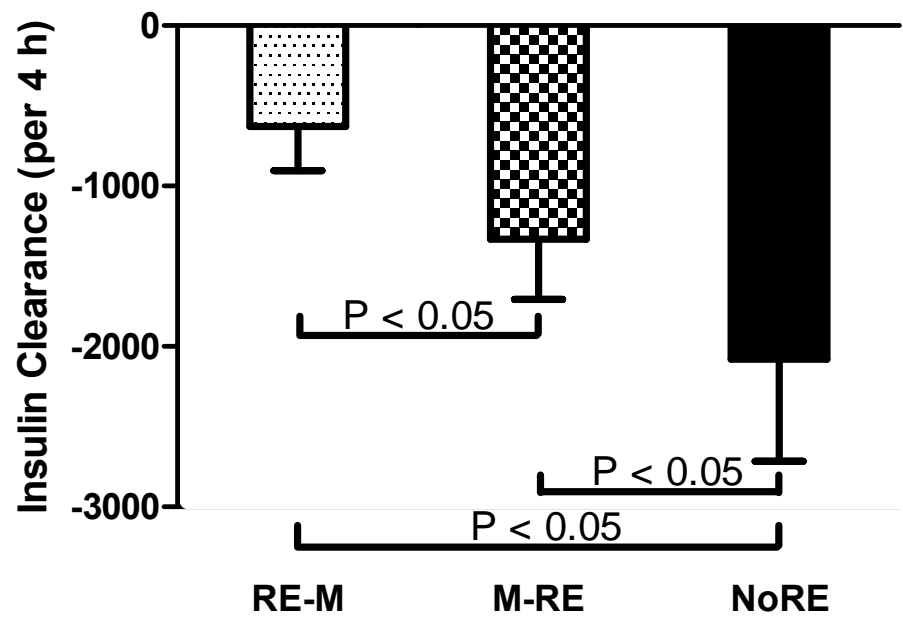

Figure 14. Insulin Clearance During Mixed Meal Testing. Values are means \pm standard error of the mean. 


\section{Glucagon Concentrations During Mixed Meal Testing}

The glucagon time course (Figure 15A), pre-dinner iAUC (Figure 15B), and post-dinner iAUC (Figure 15C) during mixed meal testing are illustrated in Figure 15. Pre-meal glucagon responses were not different between trials $(\mathrm{P}>0.05)$. The postprandial $4 \mathrm{~h}$ glucagon iAUC was significantly lower $(\mathrm{P}<0.05)$ during the $\mathrm{RE} \rightarrow \mathrm{M}$ trial compared to the other trials because glucagon increased slightly during this trial prior to the meal, and thus when the meal was eaten glucagon concentrations dropped further compared to the other trials.
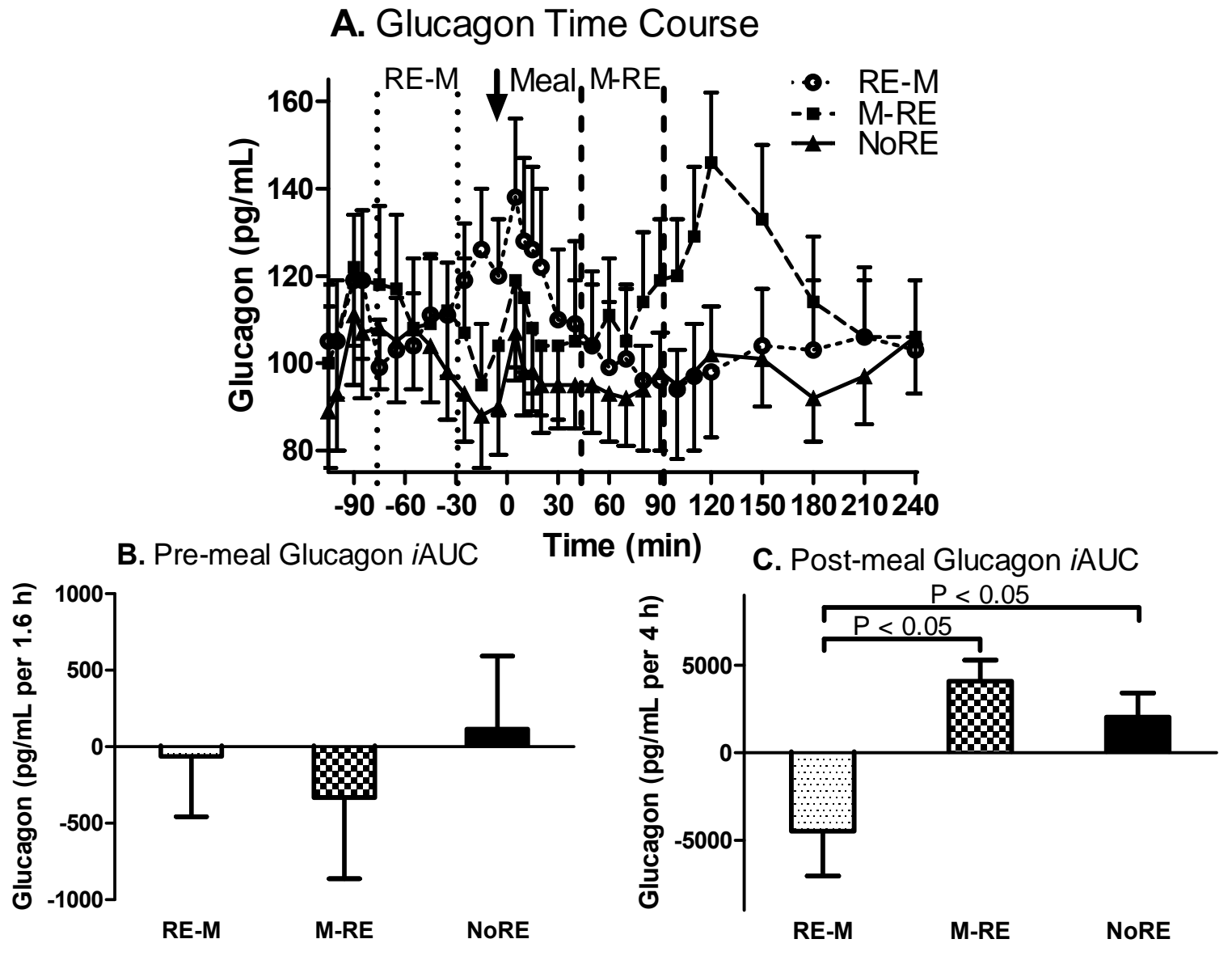

Figure 15. Glucagon Concentrations During Mixed Meal Testing.

Values are means \pm standard error of the mean. 


\section{Incretin Hormone Concentrations During Mixed Meal Testing}

The GIP time course (Figure 16A), pre-dinner iAUC (Figure 16B), and postdinner iAUC (Figure 16C) during mixed meal testing are illustrated in Figure 16. Neither pre- nor post-dinner GIP concentrations were significantly different between the different trials $(\mathrm{P}>0.05)$.
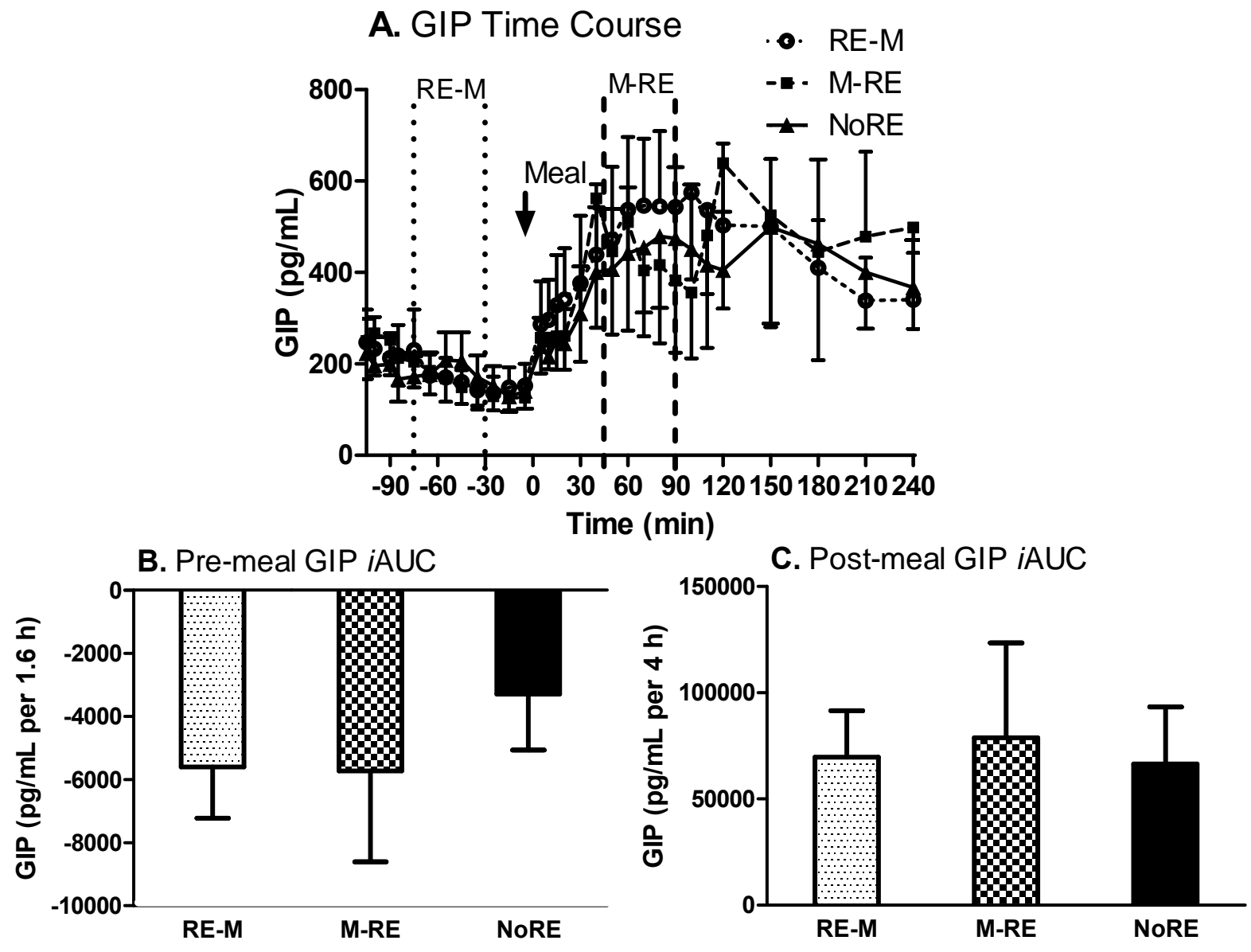

Figure 16. GIP Concentrations During Mixed Meal Testing. Values are means \pm standard error of the mean. 
The GLP-1 time course (Figure 17A), pre-dinner iAUC (Figure 17B), and postdinner iAUC (Figure 17C) during testing are illustrated in Figure 17. Pre-dinner GLP-1 concentrations were not different between trials $(\mathrm{P}>0.05)$. However, during the $\mathrm{M} \rightarrow \mathrm{RE}$ trial, the postprandial $4 \mathrm{~h}$ GLP- 1 iAUC was $50 \%$ and $49 \%$ lower compared to the $\mathrm{RE} \rightarrow \mathrm{M}$ and NoRE trial, respectfully $(\mathrm{P}<0.05)$, indicating post-dinner RE reduced postprandial GLP-1 concentrations.

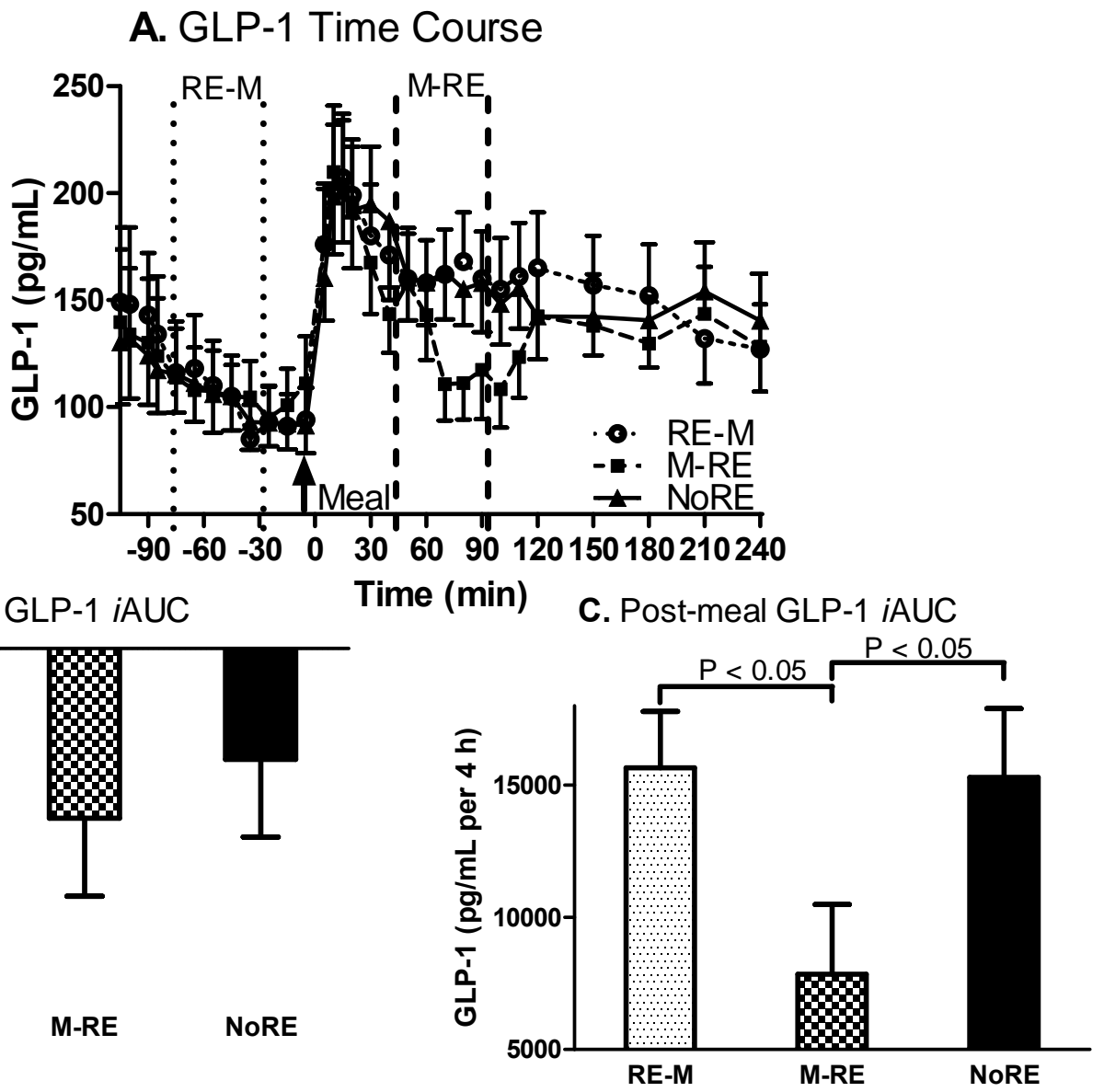

Figure 17. GLP-1 Concentrations During Mixed Meal Testing. Values are means \pm standard error of the mean. 


\section{Acetaminophen (Gastric Emptying) Responses}

The acetaminophen time course (Figure 18A) and post-dinner iAUC (Figure 18B) are illustrated in Figure 18. The $4 \mathrm{~h}$ acetaminophen iAUC, $2 \mathrm{~h}$ acetaminophen iAUC, the change in acetaminophen concentrations from 40-90 min post-meal (which corresponds to the time frame when RE was performed), or the time to peak concentration was not significantly different between trials $(\mathrm{P}>0.05)$.
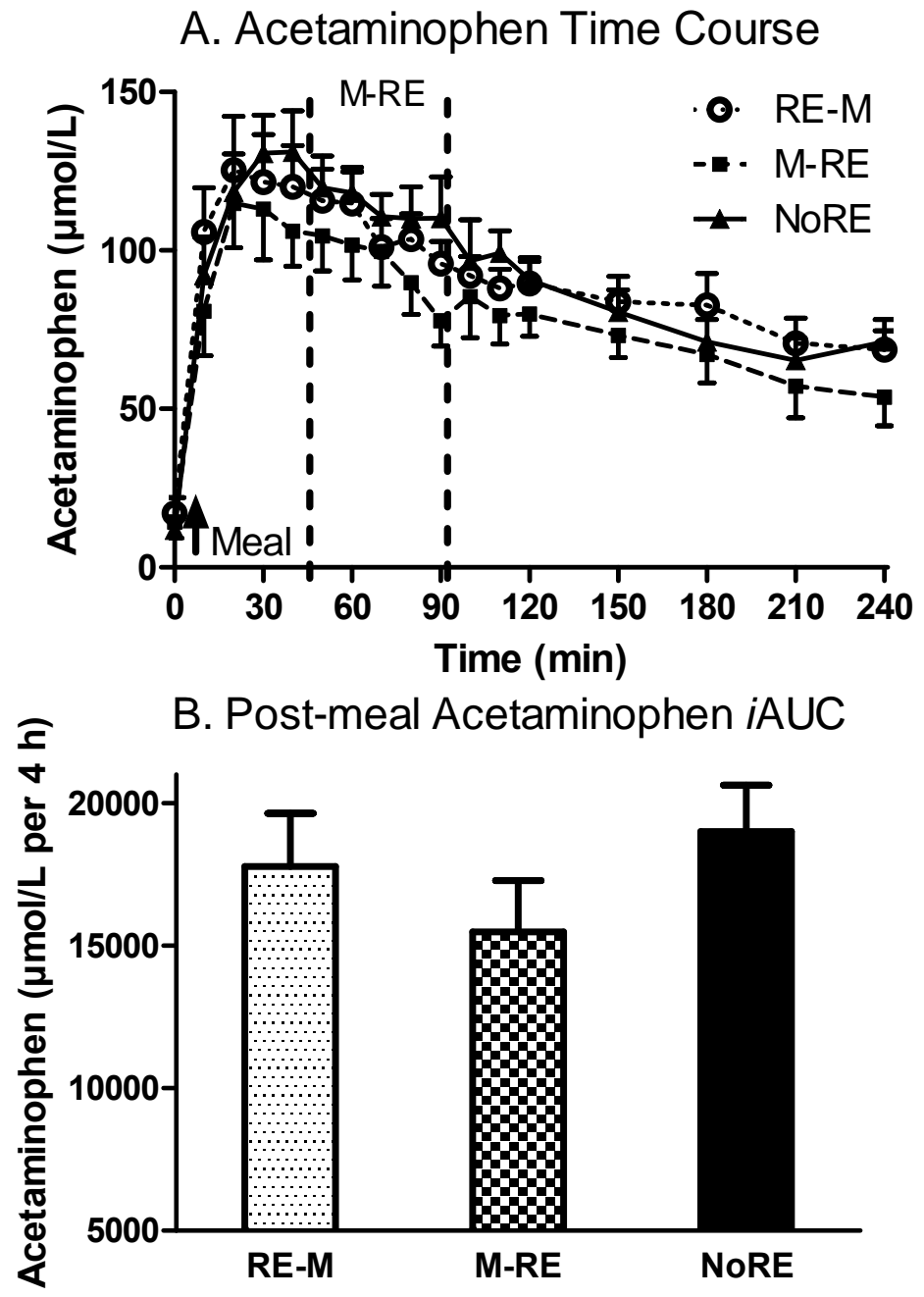

Figure 18. Acetaminophen Concentrations (Gastric Emptying) During Mixed Meal Testing.

Values are means \pm standard error of the mean. 


\section{Energy Expenditure and Whole Body Substrate Oxidation}

Table 5 lists the average energy expenditure and respiratory exchange ratio (RER) from $\sim \min 210-240$ after dinner. There were no significant differences in energy expenditure or RER between the trials.

Table 5. Energy Expenditure and Substrate Oxidation $\sim 3.5$ - 4 h After Dinner Meal

\begin{tabular}{lcc}
\hline & Energy Expenditure (kcal/min) & RER \\
\hline $\mathrm{NoRE}$ & $1.4 \pm 0.1$ & $0.85 \pm 0.01$ \\
$\mathrm{RE} \rightarrow \mathrm{M}$ & $1.4 \pm 0.1$ & $0.83 \pm 0.01$ \\
$\mathrm{M} \rightarrow \mathrm{RE}$ & $1.5 \pm 0.1$ & $0.83 \pm 0.01$ \\
\hline
\end{tabular}

Values are means \pm standard error of the mean.

$\mathrm{RER}=$ Respiratory exchange ratio (lower number $=$ greater fat oxidation, higher number = greater carbohydrate oxidation).

\section{Beta-cell Function Responses}

Table 6 lists the beta-cell function parameters during each testing trial. There were no significant differences $(\mathrm{P}>0.05)$ in beta-cell glucose sensitivity, rate sensitivity, or the potentiation factor ratio between trials.

Table 6. Beta-cell Function Parameters

\begin{tabular}{lccc}
\hline & NoRE & $\mathrm{RE} \rightarrow \mathrm{M}$ & $\mathrm{M} \rightarrow \mathrm{RE}$ \\
\hline $\begin{array}{l}\text { Beta-cell Glucose Sensitivity } \\
\left(\left(\mathrm{pmol} /\left(\mathrm{min} \mathrm{m}^{2} \cdot \mathrm{mM}\right)\right)\right.\end{array}$ & $39 \pm 5$ & $50 \pm 8$ & $50 \pm 9$ \\
$\begin{array}{l}\text { Rate Sensitivity } \\
\left(\mathrm{pmol} /\left(\mathrm{m}^{2} \cdot \mathrm{mM}\right)\right)\end{array}$ & $306 \pm 93$ & $402 \pm 141$ & $307 \pm 86$ \\
$\begin{array}{l}\text { Potentiation Factor Ratio } \\
\text { (fold) }\end{array}$ & $1.25 \pm 0.18$ & $0.87 \pm 0.08$ & $1.01 \pm 0.13$ \\
\hline
\end{tabular}

Values are means \pm standard error of the mean. 


\section{Subjective Well-being and Exercise Timing Preference}

The pre-dinner subjective well-being time course (Figure 19A), pre-dinner iAUC (Figure 19B), post-dinner time course (Figure 19C), and post-dinner iAUC (Figure 19D) are illustrated in Figure 19. The change in subjective well-being, rather than the absolute well-being value, was used because the initial (baseline) subjective well-being value was significantly different between trials $(\mathrm{P}<0.05)$, thus to better capture the change in well-being, change scores were used. Prior to the meal during the $\mathrm{RE} \rightarrow \mathrm{M}$ trial, the $1.6 \mathrm{~h}$ subjective well-being iAUC was significantly greater compared to the NoRE trial $(\mathrm{P}<0.05)$ and tended to be greater compared to the $\mathrm{M} \rightarrow \mathrm{RE}$ trial $(\mathrm{P}=0.064)$, indicating that subjective well-being increased more when the participants exercised prior to the meal compared to when they did not exercise. After the meal, the $4 \mathrm{~h}$ subjective well-being iAUC was significantly greater $(\mathrm{P}<0.05)$ during the $\mathrm{RE} \rightarrow \mathrm{M}$ and $\mathrm{M} \rightarrow \mathrm{RE}$ trials, compared to the NoRE trial, and there was no statistically significant difference between exercise trials. 
A. Pre-meal Change in Well-Being

B. Post-meal Change in Well-Being
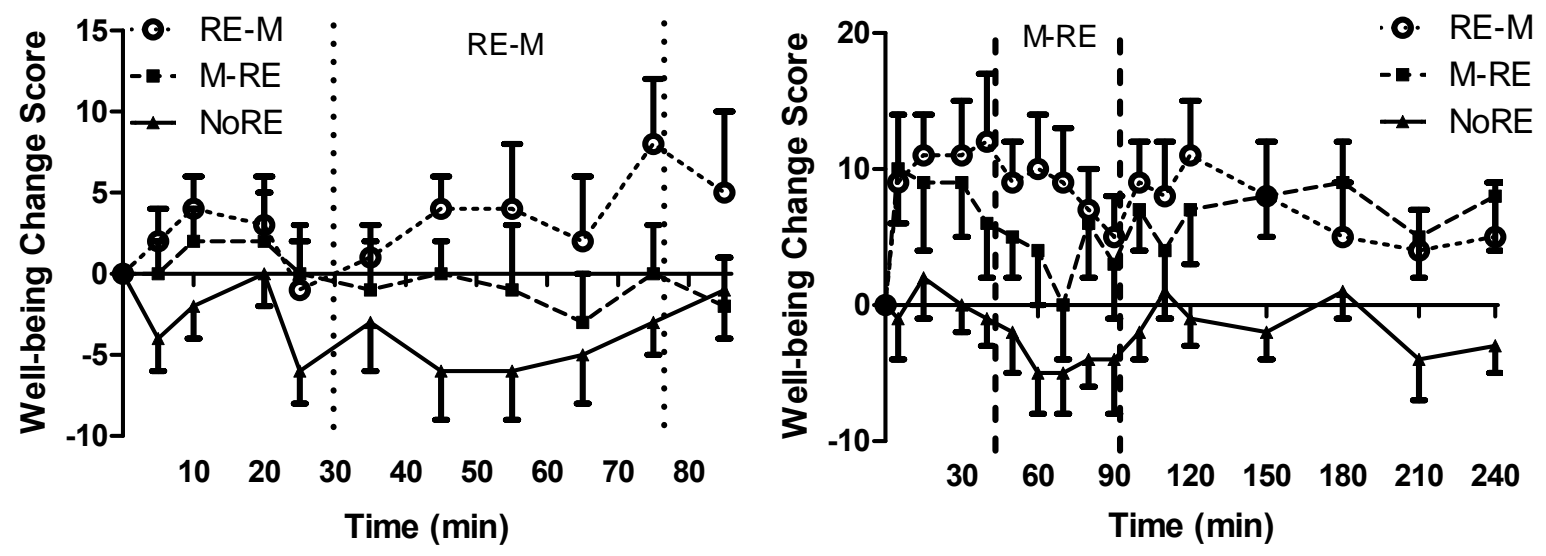

C. Pre-meal Well-being iAUC
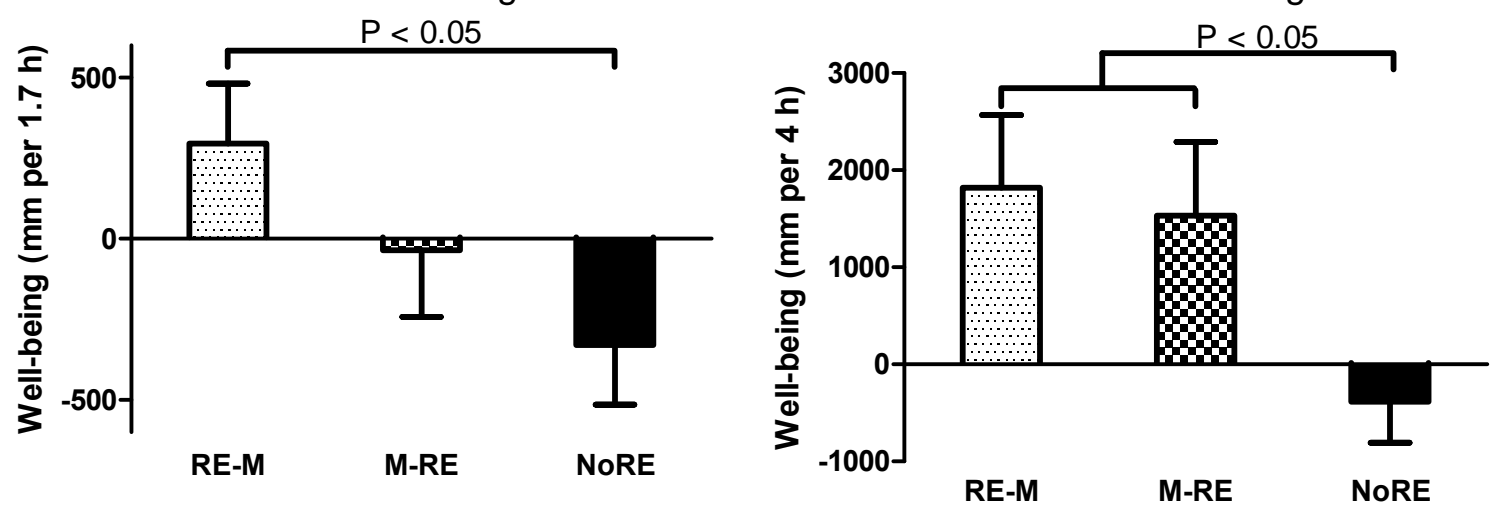

Figure 19. Subjective Well-being During Mixed Meal Testing.

Values are means \pm standard error of the mean. 


\section{Nocturnal Glucose Responses and Post-breakfast Glucose Responses}

Nocturnal and post-breakfast GGMS measured glucose responses for each trial are illustrated in Figure 20 and $\mathbf{2 1}$ and listed in Table 7. There were no significant differences $(\mathrm{P}>0.05)$ between trials for average nocturnal blood glucose concentrations, nocturnal time spent hyperglycemic or hypoglycemic, the $4 \mathrm{~h}$ post-breakfast glucose iAUC responses, or the post-breakfast time spent hyperglycemic and hypoglycemic.

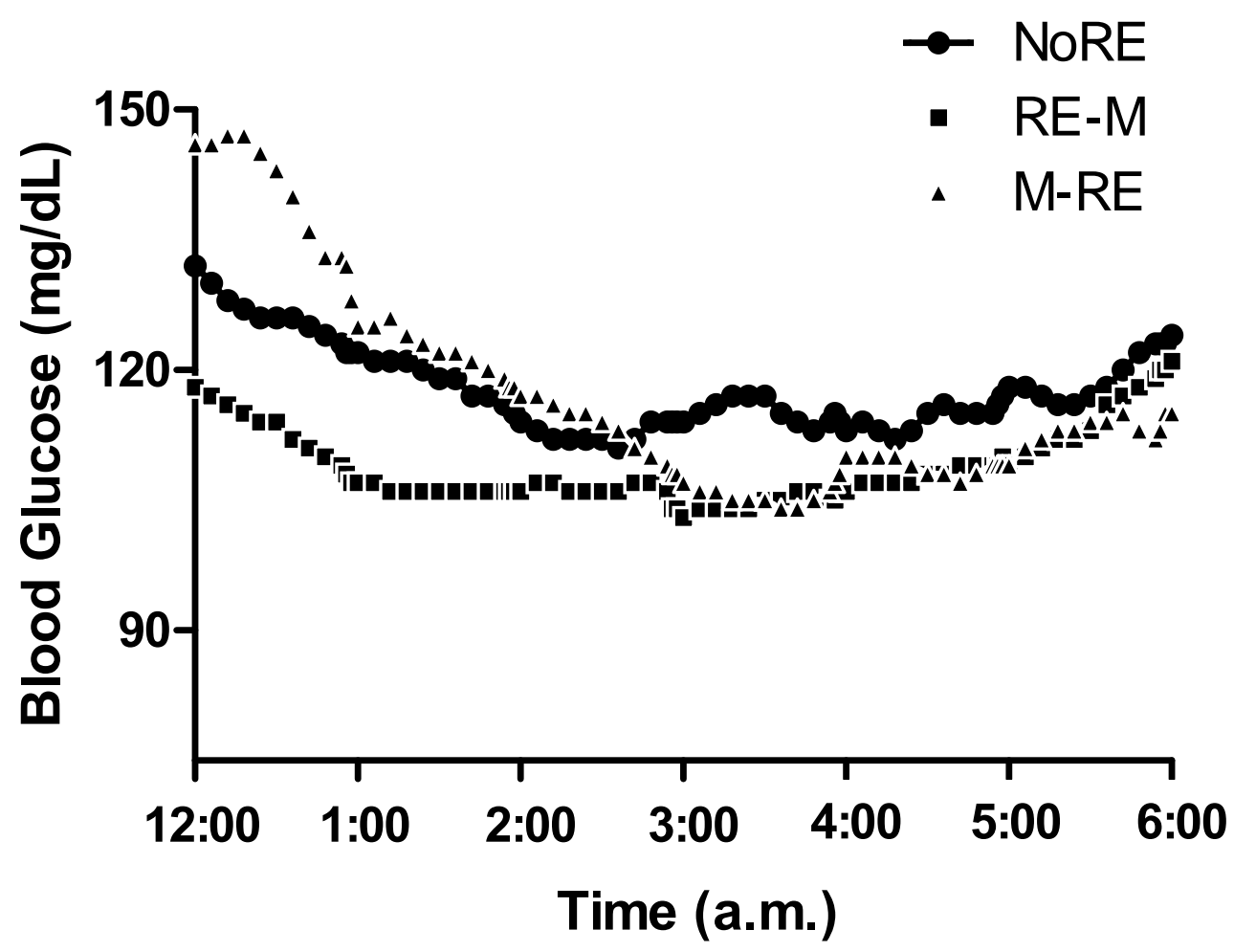

Figure 20. Nocturnal Blood Glucose Concentrations After Testing In The Lab Values are means. 


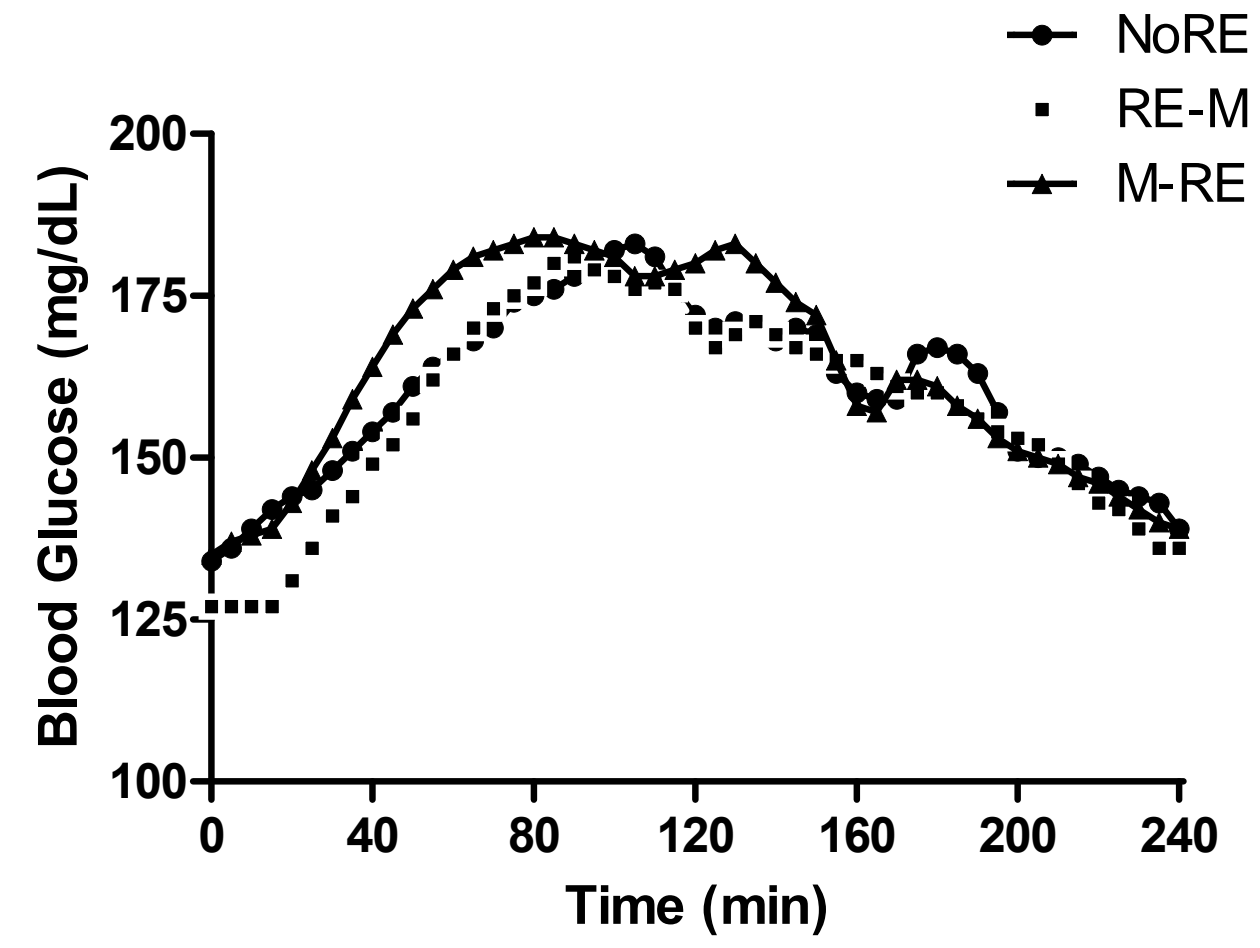

Figure 21. Postprandial Glucose Concentrations After Breakfast.

Values are means.

Table 7. Nocturnal and Post-Breakfast Glucose Responses

\begin{tabular}{lccc}
\hline & NoRE & $\mathrm{RE} \rightarrow \mathrm{M}$ & $\mathrm{M} \rightarrow \mathrm{RE}$ \\
\hline $\begin{array}{l}\text { Nocturnal Glucose Responses (12-6 a.m.) } \\
\text { Average Blood Glucose (mg/dL) }\end{array}$ & $114 \pm 9$ & $107 \pm 7$ & $115 \pm 10$ \\
$\begin{array}{l}\text { Time Hyperglycemic (min) } \\
\text { (Blood Glucose }>180 \mathrm{mg} / \mathrm{dL})\end{array}$ & $0.4 \pm 0.6$ & $2.7 \pm 3.9$ & $14.6 \pm 10.6$ \\
$\begin{array}{l}\text { Time Hypoglycemic (min) } \\
\text { (Blood Glucose }<70 \mathrm{mg} / \mathrm{dL})\end{array}$ & $26.5 \pm 30.5$ & $17.7 \pm 7.8$ & $6.9 \pm 3.7$ \\
$\begin{array}{l}\text { Post-Breakfast Glucose Responses } \\
\text { Average Blood Glucose (mg/dL) }\end{array}$ & $160 \pm 11$ & $157 \pm 12$ & $163 \pm 17$ \\
$\begin{array}{l}\text { ( h iAUC (mg/dL per 4 h) } \\
\text { Time Hyperglycemic (min) }\end{array}$ & $1288 \pm 307$ & $1486 \pm 419$ & $1356 \pm 387$ \\
$\begin{array}{l}\text { (Blood Glucose }>180 \mathrm{mg} / \mathrm{dL}) \\
\text { Time Hypoglycemic (min) }\end{array}$ & $80.8 \pm 22.7$ & $43.3 \pm 19.0$ & $66.3 \pm 27.5$ \\
(Blood Glucose $<70 \mathrm{mg} / \mathrm{dL})$ & 0 & 0 & 0 \\
\hline
\end{tabular}

Values are means \pm standard error of the mean.

The data for the nocturnal glucose response is for $\mathrm{N}=13$. Due to a malfunction in one of the CGMS during testing, data for one subject was lost thus data for post-breakfast glucose responses is for $\mathrm{N}=12$. 


\section{Glycemic Variability}

Figure 22 illustrates the $24 \mathrm{~h}$ blood glucose concentrations from the beginning of lunch on day two of each trial to the beginning of lunch on day three of each trial. Table 8 lists the average blood glucose concentrations and glycemic variability over $24 \mathrm{~h}$ between the start of breakfast on day two of each trial thru the start of breakfast on day three of each trial. There were no significant differences $(\mathrm{P}>0.05)$ for any variable measured.

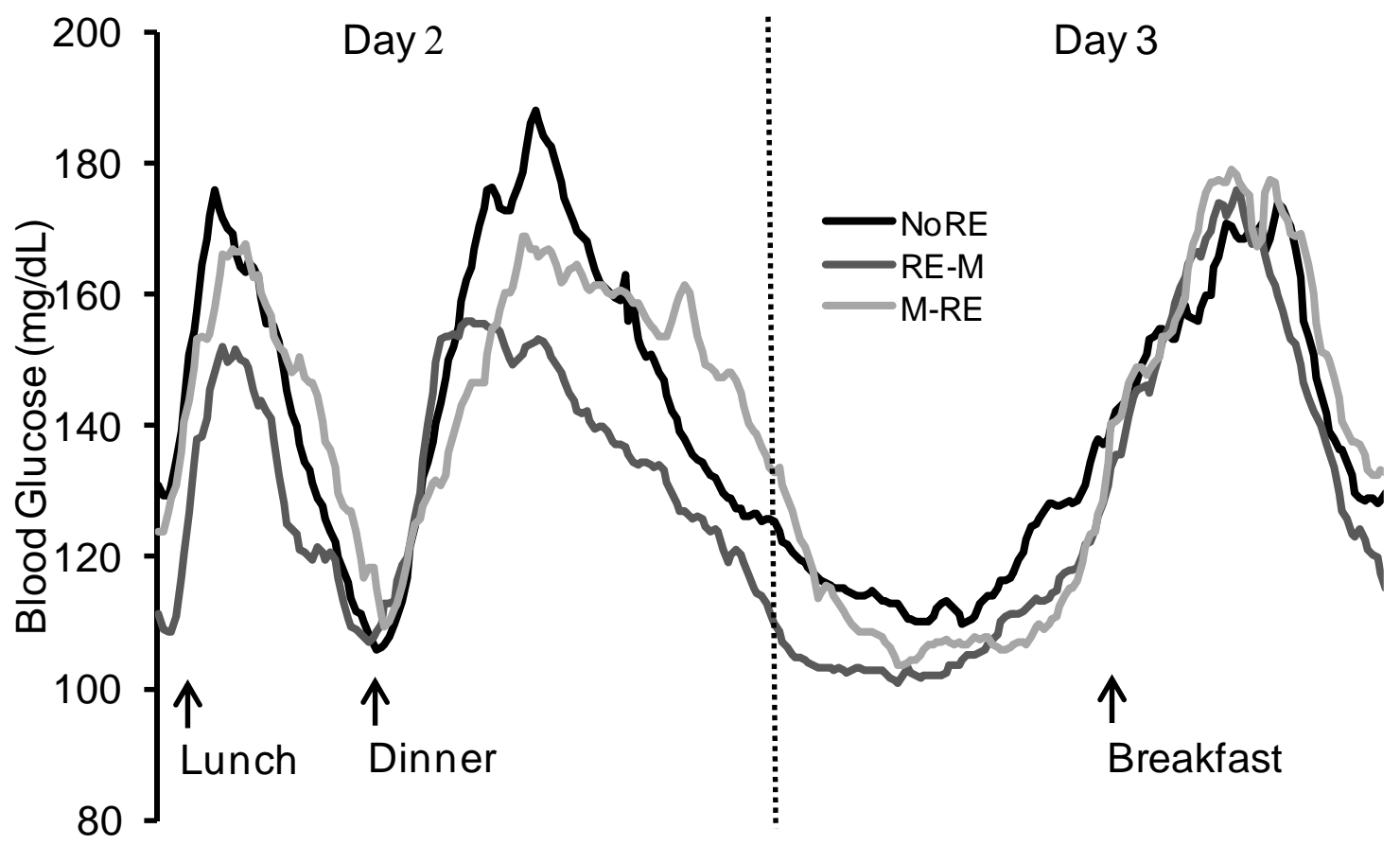

Figure 22. Blood Glucose Concentrations Over 24 Hour Period. Values are means.

Data is for $\mathrm{N}=11$ participants as 2 participants had missing data so they were excluded. 
Table 8. Average Blood Glucose and Glycemic Variability Over $24 \mathrm{~h}$

\begin{tabular}{lccc}
\hline & NoRE & RE-M & M-RE \\
\hline Average Blood Glucose (mg/dL) & $141 \pm 8$ & $130 \pm 7$ & $140 \pm 10$ \\
Standard Deviation & $33.4 \pm 3.5$ & $31.1 \pm 4.3$ & $38 \pm 4.4$ \\
CONGA-1 & $124 \pm 7$ & $115 \pm 6$ & $123 \pm 9$ \\
CONGA-2 & $112 \pm 7$ & $105 \pm 6$ & $110 \pm 8$ \\
CONGA-4 & $105 \pm 7$ & $101 \pm 6$ & $100 \pm 8$ \\
Liability Index & $973 \pm 181$ & $887 \pm 245$ & $1163 \pm 241$ \\
J-Index & $10,125 \pm 1,143$ & $8,760 \pm 1,309$ & $10,931 \pm 1,799$ \\
MAGE & $85 \pm 10$ & $82 \pm 12$ & $86 \pm 12$ \\
M-Value & $2,286 \pm 121$ & $2,116 \pm 111$ & $2,263 \pm 159$ \\
\hline
\end{tabular}

Values are means \pm standard error of the mean.

Data is for $\mathrm{N}=11$ participants as 2 participants had missing data so they were excluded.

\section{Fasting Measures The Morning Following the Acute Resistance Exercise Session}

Fasting blood glucose, insulin, c-peptide, hepatic insulin clearance, hepatic insulin resistance (HOMA-IR), whole body insulin sensitivity (QUICKI), and TAG measures are listed in Table 9. There were no significant differences between trials in any parameter measured $(\mathrm{P}>0.05)$.

Table 9. Morning Fasting Measures The Day After Testing In The Lab

\begin{tabular}{lccc}
\hline & NoRE & $\mathrm{RE} \rightarrow \mathrm{M}$ & $\mathrm{M} \rightarrow \mathrm{RE}$ \\
\hline Blood Glucose (mg/dL) & $121 \pm 7$ & $120 \pm 6$ & $120 \pm 10$ \\
Insulin (pmol/l) & $290 \pm 43$ & $256 \pm 37$ & $301 \pm 53$ \\
C-Peptide (pmol/l) & $1537 \pm 181$ & $1378 \pm 113$ & $1465 \pm 194$ \\
Insulin Clearance (\%) & $81 \pm 2$ & $82 \pm 2$ & $80 \pm 2$ \\
HOMA-IR & $12.58 \pm 1.98$ & $10.98 \pm 1.77$ & $12.05 \pm 1.92$ \\
QUICKI & $0.28 \pm 0.01$ & $0.28 \pm 0.01$ & $0.28 \pm 0.05$ \\
Triacylglycerol (mg/dL) & $134 \pm 14$ & $133 \pm 14$ & $138 \pm 16$ \\
\hline
\end{tabular}

Values are means \pm standard error of the mean. 


\section{CHAPTER 4 - DISCUSSION}

\section{Main Findings}

Knowledge of the best time to perform RE around dinner will provide health care professionals with a better idea of how to personalize RE prescription to optimize its metabolic health benefits in obese patients with T2D. Thus, the overarching goal of this project was to identify the best time, either before or after dinner, to perform RE to lower postprandial glucose and TAG concentrations, two important cardiovascular disease risk factors in obese patients with T2D. The key findings of this study are: 1) Both pre- and post-dinner RE reduces postprandial glucose concentrations, while only post-dinner RE reduces both postprandial glucose and TAG concentrations, 2) Both pre- and post-dinner $\mathrm{RE}$ reduces insulin concentrations, but via different mechanisms as pre-dinner $\mathrm{RE}$ enhances insulin clearance, whereas post-dinner RE reduces pancreatic insulin secretion and enhances insulin clearance, 3) Post-dinner RE reduces postprandial GLP-1 concentrations while pre-dinner RE does not, 4) Pre-dinner RE reduces pre-dinner FFA concentrations, 5) Gastric emptying, beta-cell function, post-dinner substrate oxidation, post-dinner GIP concentrations, and nocturnal and postprandial glucose concentrations or insulin sensitivity the following morning are not altered by acute RE, and 6) RE improved well-being when performed before or after dinner. Taken together, post-dinner RE improves metabolic control better than pre-dinner RE in obese patients with T2D, and these benefits are short lived and do not last into the overnight period or into the next day. Importantly, RE improved well-being, making it a feasible option for obese patients with T2D. 


\section{Glucose Responses}

Previous research has shown that in patients with T2D an acute bout of RE prior to a meal (s) lowers postprandial glucose concentrations [49] or improves $24 \mathrm{~h}$ glycemic control [50] within $24 \mathrm{~h}$ of the RE session, although one study showed acute RE had no impact on postprandial glucose concentrations [38]. In line with some of this previous work, in the current study the acute RE session prior to the dinner meal lowered postprandial glucose concentrations. Further, the current study is the first to examine how post-meal RE impacts postprandial blood glucose concentrations, and similar to predinner RE, post-dinner RE produced a decrease in the postprandial blood glucose iAUC. Thus, it appears that neither RE time is better compared to the other as far as lowering postprandial blood glucose concentrations.

There was tremendous inter-individual variation in the effect of $\mathrm{RE}$ on postprandial glucose concentrations in the current study with some individuals have an adverse response (i.e. increase in glucose iAUC with RE), and this has been reported by other researchers as well with aerobic exercise [36]. Bouchard et al. [82] have reported that $\sim 7 \%$ of people that exercise experience adverse responses in cardiovascular and diabetes risk factors. Further, Bouchard et al. [82]concluded that identifying the predictors of such unwarranted responses and how to prevent them will aid in better, more personalized exercise prescriptions. Interestingly, in the current study one participant had a worsening of glycemic control with pre-meal RE (adverse response), but post-meal RE improved glycemic control in this individual. In addition, two participants had a worsening of glycemic control with post-meal RE, but pre-meal RE improved glycemic control in these individuals. Taken together, these data suggest that 
the ability to improve glycemic control with acute RE may be dependent on the timing of the RE session relative to a meal in some cases. In other words, manipulating RE timing may make most people exercise responders. This data has important implications for personalized medicine, and will help make more effective exercise prescriptions that will maximize the metabolic benefits of RE in obese patients with T2D.

The postprandial glucose time course during each RE trial was drastically different. During the first 45 min after the meal, the rise in blood glucose concentrations was similar between trials. During the pre-meal RE trial, postprandial glucose concentrations were lower in the middle of the postprandial period (from $\sim 1-3 \mathrm{~h}$ ) compared to the NoRE trial. However, during the post-meal RE trial glucose concentrations were lower predominantly during exercise (from $\sim 45 \mathrm{~min}-1.5 \mathrm{~h}$ postmeal), and rebounded at the cessation of exercise to NoRE trial levels. These observations suggest that different glucose lowering mechanisms may be at play depending on the timing of RE. The rate of gastric emptying plays a large role in the postprandial glucose response, but in the current study gastric emptying was unaltered with RE, which suggests changes in gastric emptying are not responsible for reductions in blood glucose concentrations with RE. Instead, it is likely that during the pre-dinner RE trial, insulin sensitivity was increased prior to the meal which improved insulin action (an effect that may have been partially mediated by reduced pre-meal free fatty acid concentrations) in the previously exercised skeletal muscle and possibly the liver prior to ingestion of the dinner meal. This effect would increase glucose uptake into skeletal muscle and reduce hepatic glucose output to a greater extent compared to not exercising. This hypothesis is supported by the reduced insulin concentrations (thus improved insulin 
sensitivity) observed in this study. In addition, pre-meal RE probably increased postprandial skeletal muscle and liver blood flow (which is related to enhanced insulin sensitivity), which increased capillary recruitment and blood flow through previously non-flowing capillaries, thus allowing more widespread glucose uptake via the GLUT-4 transporter. This idea is supported by the work of Philips et al. [83], who showed that prior RE enhanced postprandial leg blood flow responses, compared to no exercise. During the post-meal RE trial, it is likely that the elevated insulin concentrations due to the prior meal on top of the skeletal muscle contractions worked synergistically to increase skeletal muscle blood glucose uptake, and that this mechanism at least partially contributed to reducing postprandial blood glucose concentrations during this trial. This potential mechanism is supported by the work of Larsen et al. [53] in which moderate intensity cycling exercise, beginning 45 min after breakfast, dramatically increased the rate of disappearance of labeled glucose. Once the post-dinner RE session was over, blood glucose concentrations rebounded to NoRE concentrations, and it is not clear why this occurred. It is possible that hepatic glucose output increased during RE and remained elevated immediately after exercise for a short time while glucose uptake into skeletal muscle was reduced because of the cessation of muscle contractions and the lower insulin concentrations. In support of this, Larsen et al. [53] showed a similar trend such that moderate intensity cycling exercise, beginning 45 min after breakfast, also resulted in a rebound glycemia effect once exercise stopped that was associated with a slight increase in the rate of appearance of $3-\left[{ }^{3} \mathrm{H}\right]$ glucose and a slight decrease in the rate of disappearance of $3-\left[{ }^{3} \mathrm{H}\right]$ glucose immediately after the cessation of post-meal exercise. 


\section{Triacylglycerol Responses}

For the first time, this study assessed how RE impacts post-meal total TAG concentrations in obese patients with T2D. The data suggests that post-meal RE is better compared to pre-meal RE at lowering postprandial total TAG concentrations. Although no study has directly compared pre-meal exercise to post-meal exercise on the postprandial total TAG response in patients with $\mathrm{T} 2 \mathrm{D}$, the available data with $\mathrm{AE}$ is in line with this finding. For example, in two studies $[37,40]$ the AE session was performed the day prior to the test meal given and in these studies there was no change in postprandial total TAG concentrations with AE, compared to a no exercise trial. Interestingly, in another study the AE bout was performed starting 90 min after a high fat breakfast meal, and in this study the exercise bout significantly reduced the average postprandial total TAG response compared to no exercise [48].

Clearly, the data suggest that exercise timing is of critical importance and that post-meal RE is better compared to pre-meal RE at lowering postprandial total TAG concentrations in most patients with T2D. Although some individuals had benefits in postprandial TAG with both RE times, in $7 / 13$ people post-meal RE was better at lowering postprandial TAG concentrations compared to pre-meal RE, while pre-meal RE was better (and only slightly) in 3 / 13 people compared to post-meal RE. Interestingly, in three individuals, neither pre-meal nor post-meal RE improved postprandial TAG concentrations and instead these individuals had greater postprandial TAG concentrations with RE.

The majority of TAG in circulation originates from either exogenous sources and is in the form of chylomicron particles or endogenous sources from the liver and is in the 
form of VLDL-TAG [84]. In the current study, both particles were measured to gain an understanding of which source of TAG RE modifies. Neither pre-meal nor post-meal RE modified postprandial chylomicron or VLDL-2 TAG particles. Instead, the reduction in postprandial total TAG concentrations with post-dinner RE was primarily mediated by reduced VLDL-1 TAG concentrations. The mechanism(s) for the reduction in VLDL-1 TAG with RE was likely mediated by enhanced hydrolysis of TAG by lipoprotein lipase in skeletal muscle and/or by reduced VLDL-1 TAG secretion by the liver.

\section{Insulin Secretion and Clearance}

In the current study, both pre-dinner RE and post-dinner RE reduced postprandial insulin concentrations, and this finding is in line with other work demonstrating acute RE $\sim 18 \mathrm{~h}$ prior to a meal reduces postprandial insulin concentrations (thus improves insulin sensitivity) in individuals with T2D [38]. The amount of insulin in circulation is dependent on the amount of insulin secreted from the $\beta$-cells of the pancreas in addition to the amount of insulin cleared from the circulation (which can occur in any organ or tissue with an insulin receptor). With pre-dinner RE, the reduction in postprandial insulin concentrations was mediated primarily by enhanced insulin clearance, and this finding is similar to previous work [38]. During the post-dinner RE trial, we show for the first time that the reduction in insulin concentrations was mediated by both increased insulin clearance and reduced insulin secretion, although the increase in insulin clearance was not as great as compared to the pre-dinner RE trial.

Pancreatic $\beta$-cell insulin secretion is modulated primarily by blood glucose concentrations, thus reduced blood glucose concentrations likely mediated the reduction in insulin secretion with post-dinner RE. The mechanism by which RE increases insulin 
clearance is not understood, but one potential explanation may be increased skeletal muscle and/or liver blood flow. Increased blood flow in these tissues may increase blood flow through previously non-flowing capillaries, allowing more insulin to be exposed to and bind to the insulin receptor, internalized, and degraded. This hypothesis is supported by the work of Philips et al. [83] who showed that prior RE enhanced postprandial leg blood flow responses, compared to no exercise. Another possibility may by that RE reduced portal vein glucose and/or FFA concentrations. Given that glucose and FFA have been shown to independently and synergistically impair insulin binding to receptors, it is possible that a RE induced reduction in these substrates in the portal vein allowed more hepatic insulin clearance [85]. However, these mechanisms are only speculative and warrant further investigation.

\section{Incretin Hormone Responses}

The incretin hormones GIP and GLP-1 are primarily secreted from the small intestine in response to nutrient ingestion and help regulate postprandial glucose and TAG concentrations via different mechanisms. The current study is the first to examine how RE timing impacts postprandial incretin hormone concentrations in obese patients with T2D. The data suggest that RE, independent of its timing, does not alter GIP concentrations, thus it is likely that GIP did not play a role in the reduction of insulin or TAG concentrations with RE. Similarly, in another study after 28 days of metformin or placebo treatment, AE performed prior to a lunch meal did not alter postprandial GIP responses in patients with T2D [86].

In the current study, pre-dinner RE had no effect on postprandial GLP-1 concentrations. This finding is in line with previous research, where AE performed prior 
to a lunch meal did not alter postprandial GLP-1 responses in patients with T2D [86]. On the contrary, in the current study post-dinner RE significantly attenuated postprandial GLP-1 concentrations, and this effect may have in part contributed to reduced insulin secretion (along with reduced glucose concentrations). Interestingly, post-meal AE has been shown to accentuate the rise in postprandial GLP-1 concentrations in healthy patients [62], whereas in the current study post-dinner RE reduced postprandial GLP-1 concentrations. The discrepancy in findings between studies is not clear, but may be due to different study designs, mode of exercise, different hormonal milieu induced by RE, and different populations used.

\section{Beta-cell Function}

The function of a beta-cell is to release biologically active insulin into the blood in response to glucose concentrations. Typically beta-cell function is assessed by the sensitivity of the beta-cell to glucose, or the insulin secretion rate at a given blood glucose concentration. The effect of RE on beta-cell function is mixed. One study demonstrated resistance training improved beta-cell function in individuals without T2D [87]. On the contrary, resistance training did not alter beta-cell function in overweight Latino adolescent males without T2D [88] or in patients with T2D [89]. In the current study, neither beta-cell glucose sensitivity, rate sensitivity, nor insulin potentiation were altered with pre- or post-dinner RE in the current study. Although insulin secretion was reduced with post-dinner RE in the current study, this was not due to reduced beta-cell function but rather reduced glucose concentrations. 


\section{Subjective Well-being}

One secondary goal of this study was to determine how the different RE times altered subjective well-being (pleasure) to determine how feasible each RE time is (i.e. did the participants actually enjoy exercising before or after they ate compared to not exercising). Importantly, the data suggest that patients with T2D feel better after a meal when RE is performed either before or after the meal, and that post-dinner RE does not deteriorate subjective-well-being, making it a feasible option. Interestingly, when the participants were asked "Did you like exercising before or after dinner better?", 11 out of 13 participants preferred RE before dinner. Thus, although post-dinner RE is feasible and does not deteriorate subjective well-being, many patients may not be motivated to exercise at this time.

\section{Nocturnal and Post-breakfast Glucose Responses and Insulin Sensitivity the Next}

\section{Day}

Previous research has shown that acute RE either in the morning prior to lunch or prior to dinner does not alter average nocturnal blood glucose concentrations $[39,50]$ and our findings are in agreement with these studies. In addition, our study shows that changing the RE timing to after dinner and closer to the nocturnal period does not modulate nocturnal blood glucose concentrations when measured by CGMS. On the contrary, van Dijk et al. [50] reported that an acute bout of RE reduced the average time spent hyperglycemic (blood glucose $>180 \mathrm{mg} / \mathrm{dL}$ ) during the overnight period and reduced average morning (6 a.m. - 12 p.m.) glucose concentrations and time spent hyperglycemic, whereas we did not observe such a finding. A potential reason for the discrepancy in findings between studies could be due to differences in populations and 
their glycemic control. The subjects in the van Dijk et al. [50] study had an average baseline fasting blood glucose of $\sim 177 \mathrm{mg} / \mathrm{dL}$, an average $\mathrm{HbA} 1 \mathrm{c}$ of $7.5 \%$, and during the no exercise trial had an average nocturnal blood glucose concentrations that was $\sim 160$ $\mathrm{mg} / \mathrm{dL}$, were hyperglycemic overnight for an average of $\sim 1 \mathrm{~h}$ and $42 \mathrm{~min}$, and had an average morning period (6 a.m. - 12 p.m.) glucose concentrations of $\sim 155 \mathrm{mg} / \mathrm{dL}$, whereas the subjects in our study had an average baseline fasting blood glucose of $\sim 148$ $\mathrm{mg} / \mathrm{dL}, \mathrm{HbAlc}$ of $7.2 \%$, and during the no exercise trial had an average nocturnal blood glucose concentration of $\sim 119 \mathrm{mg} / \mathrm{dL}$ and spent on average $\leq 16 \mathrm{~min}$ hyperglycemic, and had an average morning period (6 a.m. - 12 p.m.) glucose concentrations of $\sim 151 \mathrm{mg} / \mathrm{dL}$. Therefore, the participants in our study were better controlled diabetics compared to the participants in the van Dijk et al. [50] study, thus it is possible the patients in our study had less room for improvement.

In the current study neither pre- nor post-dinner RE altered subsequent postprandial glucose responses to a mixed composition breakfast meal the following morning ( $\sim 12-15 \mathrm{~h}$ later). This finding is similar to an earlier study that reported $\mathrm{RE}$ performed $\sim 18 \mathrm{~h}$ prior to an oral glucose tolerance test does not improve postprandial glucose responses [38] but is not in agreement with another study that showed acute RE within $24 \mathrm{~h}$ of an oral glucose tolerance test reduced postprandial glucose concentrations [49]. One potential reason for the lack of change in glycemic control in the current study could be related to the lack of change in insulin sensitivity the following morning. In the current study, whole body RE did not increase or decrease fasting blood glucose or insulin concentrations or whole body insulin sensitivity (QUICKI) or hepatic insulin resistance (HOMA-IR) 12-15 h later. Likewise, Gordon et al. [68] reported that an acute 
session of lower body RE did not modulate whole body insulin sensitivity (HOMA2) 24, 48 , or $72 \mathrm{~h}$ later in type 2 diabetic patients. In addition, in the current study basal hepatic insulin clearance was not different between trials. On the contrary, using an oral glucose tolerance test others have found that prior RE within $24 \mathrm{~h}$ has reduced postprandial insulin concentrations, suggesting improved insulin sensitivity [38, 49].

Independent of timing, whole body RE did not alter average $24 \mathrm{~h}$ blood glucose concentrations or glycemic variability in this study, and this finding is in agreement with some [39] but not all research [50]. The lack of change in $24 \mathrm{~h}$ glycemic variability could be attributed to the fact that the whole body RE session in the current study was relatively low in energy expenditure (average of $\sim 75-77$ Calories above resting energy expenditure) and did not improve insulin sensitivity the next day. It may be that a greater energy deficit is needed to improve average $24 \mathrm{~h}$ blood glucose concentrations and glycemic variability in this population.

\section{Conclusion}

In conclusion, acute post-dinner RE improves postprandial glucose and TAG concentrations better than pre-dinner RE in obese patients with T2D. Future studies are needed to determine if long-term post-meal exercise training in patients with T2D would better improve metabolic health and reduce cardiovascular disease more so than pre-meal exercise.

\section{Limitations}

This project has several limitations including:

1. Acetaminophen was used to indirectly measure gastric emptying instead of the "gold standard" scintigraphy method. However, when validated with scintigraphy, 
acetaminophen has been shown to yield similar results about gastric emptying [90].

2. Tracers were not used to determine if post-meal RE reduces exogenous or endogenous glucose or TAG rate of appearance and/or increases its rate of disappearance.

3. Numerous hormones may be altered with RE, but we only measured hormones that are most critical in glycemic and lipemic control and gastric emptying.

4. The responses to an acute RE session are being assessed prior to adaptive changes that may take place after weeks of RE.

5. Insulin secretin or clearance was not directly measured using arteriovenous differences.

6. The beta-cell function model used in this study was developed using Caucasian participants and has never been validated using other populations. The present study included a mix of Caucasian, African American, and Hispanic patients.

\section{Future Directions}

This is the first study to examine how the timing of RE relative to a meal alters glycemic and lipemic control in patients with T2D. This study can serve as a stepping stone to several potential future studies with the goal of delineating the best time to perform RE in patients with T2D. Future studies could be:

1) A training study to determine how RE timing over a longer duration impacts clinical markers of glycemic control, such as HbAlc, or lipemic control, such as HDL-C, in patients with T2D. 
2) A study to determine how RE at different times of the day impacts glycemic or lipemic control in patients with T2D (i.e. morning RE vs. afternoon RE vs. evening RE).

3) A study to determine how RE at different times before or after a meal impacts postprandial glycemia and lipemia in patients with T2D (i.e. $30 \mathrm{~min}$ vs. $60 \mathrm{~min}$ vs $1.5 \mathrm{~h}$ vs $2 \mathrm{~h}$ before or after a meal). 


\section{CHAPTER 5 - EXTENDED LITERATURE REVIEW}

This literature review will first describe the regulation of blood glucose, TAG, and glucoregulatory hormone concentrations in healthy and in patients with T2D. Then the links between abnormally high blood glucose and TAG concentrations and cardiovascular disease will be discussed. Lastly, the impact of AE and RE on metabolic control, gastric emptying, endocrine responses, and beta-cell function in non-diabetic and in patients with T2D will be discussed.

\section{Hormonal Regulation of Glycemia}

The regulation of postprandial glycemia is tightly controlled via hormones released from the intestine and endocrine pancreas. The endocrine pancreas secretes five different hormones including insulin, amylin, glucagon, somatostatin, and pancreatic polypeptide, but the hormones most critical for controlling blood glucose are insulin and glucagon. Insulin is secreted from the pancreatic $\beta$-cells in response to a meal and its functions include stimulating glucose uptake into skeletal muscle and adipose tissue [91], reducing hepatic glucose secretion [92], and increasing TAG hydrolysis by increasing adipose tissue lipoprotein lipase activity [93]. Insulin's antagonist, glucagon, is secreted from pancreatic $\alpha$-cells in response to hypoglycemia and its primary function is to stimulate hepatic glucose output [94].

Both insulin and glucagon secretion are modulated by the gut derived incretin hormones GIP and GLP-1. GIP is secreted from endocrine K-cells of the duodenum and jejunum of the small intestine in response to macronutrient ingestion, and this hormone potentiates insulin secretion in a glucose dependent manner, inhibits glucagon secretion, and stimulates fatty acid synthesis and re-esterification in adipose tissue [95]. GLP-1 is 
secreted predominately from endocrine L-cells located in the ileum and colon of the intestine in a biphasic manner in response to nutrient ingestion, and its functions include inhibiting gastric acid secretion and gastric emptying while stimulating insulin release in a glucose-dependent fashion [96].

\section{Gastric Emptying and Post-meal Glycemia}

It is now recognized that the rate of gastric emptying has a major influence on the postprandial glycemic response. The rate of gastric emptying is controlled by electrical slow waves generated by the interstitial cells of Cajal of the stomach. Gastric emptying is modulated by neural input and by the gut hormones GLP-1 [63-65] and cholecystokinin (CCK) [97]. The relationship between gastric emptying and glycemia was shown in a series of studies in which glucose was infused directly into the duodenum (to control gastric emptying) at different rates (i.e. between 1-4 Cal/min). In healthy young patients [98], healthy older patients, and patients with T2D [99], a higher rate of glucose infusion into the duodenum $(2-4 \mathrm{Cal} / \mathrm{min})$ resulted in significantly greater blood glucose concentrations compared to saline or $1 \mathrm{Cal} / \mathrm{min}$ glucose infusions. However, there was no difference in the glycemic response between the 2 and $4 \mathrm{Cal} / \mathrm{min}$ infusion, which likely occurred because of the much greater insulin response to counteract the hyperglycemia with a $4 \mathrm{Cal} / \mathrm{min}$ infusion. Gonlachanvit et al. [100] showed that accelerating gastric emptying with erythromycin infusion increased the postprandial glucose response, whereas slowing gastric emptying with morphine infusion lowered the postprandial glycemic response in T2D. In another study, reduced gastric emptying (mediated by i.v. infusion of GLP-1 prior to a meal), was associated with reduced postprandial glycemia and lipemia in healthy male volunteers [101]. Taken together, these studies show that 
modulation of gastric emptying plays a role in determining the postprandial glycemic response.

\section{Impaired Hormonal Regulation of Glycemia in Type 2 Diabetics}

Impaired hormonal regulation of glycemia plays a major role in fasting and postprandial hyperglycemia in T2D [102]. There is evidence that, compared to healthy age, sex, and weight matched control subjects, patients with T2D are insulin resistant and have elevated rates of both endogenous and exogenous glucose release into circulation [103-108] in addition to impaired exogenous and endogenous glucose clearance [105106]. The latter is controversial as not all studies demonstrate impaired glucose clearance in T2D [107-108].

In the early stages of T2D when insulin secretion in response to a meal still occurs (prior to $\beta$-cell failure), elevated fasting and postprandial glucose concentrations appear to be mediated by a combination of abnormalities including 1) hepatic insulin resistance resulting in failure of insulin to inhibit hepatic glucose release, gluconeogenesis, and glycogenolysis [108-110], 2) failure of glucagon suppression in the postprandial period, resulting in a lower insulin to glucagon ratio, which contributes to excessive endogenous glucose release and hepatic gluconeogenesis and glycogenolysis [108, 111], and 3) impaired ability of insulin to stimulate vasodilation in skeletal muscle [56], thus limiting postprandial blood glucose uptake in skeletal muscle which contributes to postprandial hyperglycemia in diabetes. In the later stages of $\mathrm{T} 2 \mathrm{D}$, insulin secretion from pancreatic $\beta$ cells ceases ( $\beta$-cell failure), and thus hypoinsulinemia contributes substantially to hyperglycemia in advanced T2D [112]. 
In addition to impaired insulin and glucagon responses in T2D, there is also evidence that the incretin effect is impaired. For instance, patients with T2D have lower postprandial GLP-1 concentrations compared to non-diabetic obese patients after ingestion of a breakfast meal. This suggests that impaired GLP-1 responses contribute to delayed and blunted insulin responses and lack of suppression of postprandial glucagon in patients with T2D [113-115]. In support of this hypothesis, Vilsboll et al. [116] reported that GLP-1 infusion augmented late phase (20-120 min) insulin secretion in response to glucose in patients with $\mathrm{T} 2 \mathrm{D}$ to a level comparable to non-diabetic patients. In contrast, GIP infusion did not alter late phase insulin secretion to glucose in patients with T2D, suggesting a defect in late phase amplification of insulin secretion by GIP [116]. In addition, the blunted GLP-1 response in T2D may contribute to the higher rates of gastric emptying in some of these patients, which may further worsen postprandial hyperglycemia [117-119]. However, the rate of gastric emptying in patients with T2D is highly variable, and in the later stages of T2D abnormally delayed gastric emptying or diabetic gastroparesis may exist. This delayed gastric emptying of a meal may contribute to postprandial hypoinsulinemia in these patients, although this hypothesis warrants further investigation.

\section{Consequences of Hyperglycemia}

Diabetes is an independent risk factor for vascular disease [120-121], and there is strong epidemiological and experimental evidence that hyperglycemia contributes to the increased risk of vascular disease with diabetes. For example, patients with type 1 diabetes suffer from hyperglycemia due to a genetic defect in pancreatic $\beta$-cells resulting in inadequate insulin production, and these patients have much higher rates of 
cardiovascular disease compared to age-matched non-diabetics [122-123]. In addition, other association studies have reported that postprandial hyperglycemia and glycemic peaks measured $2 \mathrm{~h}$ after a glucose load [124-130] are better predictors of cardiovascular disease in non-diabetic and diabetic patients compared to fasting blood glucose concentrations.

The molecular mechanisms by which postprandial hyperglycemia promotes vascular disease are complex and involve several distinct but interrelated mechanisms. Nitric oxide (NO) is a gas molecule that is generated from the metabolism of the amino acid L-arginine by the enzyme endothelial NO synthase (eNOS). The function of NO is to relax smooth-muscle cells, which prevents leukocyte adhesion, platelet adhesion, and aggregation in the arterial wall [131]. There is evidence that chronic hyperglycemia impairs endothelial function in isolated aortas of animals [132-134] and in-vivo in humans [135-136]. Hyperglycemia inhibits the production of the vasodilator NO, thus reduced NO mediates some of the effects of hyperglycemia on endothelial function [137140].

In addition, hyperglycemia impairs endothelial function by promoting an imbalance in oxidative stress and antioxidant defenses. There is in vitro [141] and in vivo [142-143] evidence showing that excessive oxidative stress impairs endothelial cell function in diabetes, and that the acute effects of hyperglycemia on endothelial dysfunction are blunted by antioxidants.

Advanced glycosylation end products (AGE) are also responsible for the accelerated vascular disease in diabetes [144]. In the arterial walls, non-enzymatic reactions between glucose and proteins or lipoproteins result in the formation of AGE 
which accelerate vascular disease via receptor independent and receptor dependent mechanisms. One of the most studied receptor independent mechanisms by which AGE promote atherosclerosis include the glycosylation of the apolipoprotein B [145] and phospholipid component of LDL [146], which alters the function of LDL and makes it more susceptible to oxidation, thus increasing its atherogenicity. The receptor dependent effects of AGE are mediated through the AGE receptor (RAGE). Research has shown that AGE interaction with RAGE on endothelial cells increases oxidative stress [147-148] while reducing endothelial barrier function and increasing endothelial barrier permeability [149]. This allows more lipid entry into the subendothelium, which contributes to the fatty streak of atherosclerotic lesions, whereas blockade of the AGERAGE interaction in diabetic mice suppressed the fatty streaks in complex atherosclerotic lesions [150].

Hyperglycemia induced increases in inflammatory molecules may also contribute to the increased risk of vascular disease in patients with T2D. Hyperglycemia has been shown to activate vascular smooth muscle nuclear factor of activated t-cells (NFAT), a proinflammatory cytokine that exacerbates atherosclerosis and restenosis [151].

Taken together, there is strong evidence that hyperglycemia plays a significant role in the increased risk of vascular disease in diabetes via multiple different mechanisms. Thus, the main goal in the treatment of T2D includes lowering blood glucose levels.

\section{Regulation of Triacylglyceridemia}

The majority of TAG in circulation are bound to lipoproteins [84]. Exogenous TAG from the diet are not absorbed as TAG but are first digested by gastric lipases into 
fatty acids and monoglycerides, particles that are more readily absorbed in the proximal jejunum and some distal parts of the small intestine [152]. Once in an enterocyte, the absorbed fatty acids and monoglycerides are re-synthesized into triacylglycerol's and incorporated into a chylomicron lipoprotein and secreted into intestinal lymph and eventually the blood [152]. In humans, the chylomicron particle is very large, has a lower density compared to other lipoproteins, and is composed primarily (80-95\%) of triacylglycerol [84]. Chylomicrons in the blood are hydrolyzed into fatty acids by lipoprotein lipase in the capillary beds of adipose tissue and muscle and then taken up by the tissue [84].

The other primary source of TAG in circulation is endogenous TAG secreted from hepatocytes as part of very-low density-lipoprotein (VLDL) particles [84]. These particles are much denser than chylomicrons but contain less TAG (roughly $55-80 \%$ of the particle is TAG) [84]. These TAG particles are assembled in the endoplasmic reticulum of hepatocytes, and the fatty acids that make up these TAG particles come from a combination of sources including free fatty acids from circulation and de novo synthesized fatty acids in the hepatocyte [84]. VLDL particles can be categorized into two subclasses including VLDL-1 and VLDL-2 [153]. VLDL-1 particles contain more TAG and are less dense (Svedberg flotation rate, Sf 60-400) compared to VLDL-2 particles (Sf 20-60), which contain more cholesterol [153]. Interestingly, VLDL TAG are cleared via the same lipolytic pathway as chylomicron TAG (i.e. via lipoprotein lipase), although lipoprotein lipase has a much greater affinity for chylomicron TAG than VLDL TAG [154]. 
The TAG in circulation can also be bound to intermediate density lipoproteins, low density lipoproteins, high density lipoproteins, or as free TAG [84]. However, it is thought that these TAG concentrations are relatively low compared to chylomicron and VLDL TAG [84].

Hormones play a role in the regulation of plasma TAG concentrations. For instance, insulin has been shown to increase lipoprotein lipase activity in adipose tissue [93] and inhibit its activity in skeletal muscle [155], thus affecting the distribution and clearance of TAG from circulation into tissues. The incretin hormone GIP has been shown to increase adipose tissue lipoprotein lipase activity [156]. In the liver, acute insulin infusion inhibits VLDL-1 secretion but has no effect on VLDL-2 secretion [157]. Estrogen treatment in post-menopausal women has been shown to stimulate VLDL-1 secretion [158].

\section{Consequences of Hypertriacylglyceridemia}

Although T2D is characterized by hyperglycemia, these patients are dyslipidemic as well and have elevated postprandial TAG concentrations [48]. Epidemiological studies suggest that elevated non-fasting TAG levels are associated with increased risk of myocardial infarction, ischemic heart disease or stroke, and death in men and women [159-162]. Mechanistic studies show that elevated postprandial TAG concentrations, primarily due to elevated VLDL TAG concentrations [163], increase the risk of cardiovascular disease via direct and indirect mechanisms. Excessive TAG concentrations directly interfere with the function of progenitor cells [164], induce apoptosis in endothelial cells [165], increase inflammation [165], increase circulating endothelial cell microparticles [166], and reduce endothelial function [167], all processes 
that contribute directly to cardiovascular disease. In addition, excessive TAG concentrations suppress the protective effect of high-density lipoprotein cholesterol [168]. Thus, in addition to hyperglycemia, hypertriacylglyceridemia contributes to the increased risk of vascular disease in T2D.

\section{Acute Aerobic Exercise and Glycemic Control in Type 2 Diabetes}

Aerobic exercise has traditionally been the exercise mode prescribed to patients with T2D. To my knowledge, Larsen et al. [53] published the first study examining how acute $\mathrm{AE}$ impacts postprandial blood glucose in response to a mixed meal in T2D. In this study nine patients with T2D completed three testing trials including an exercise trial, a no exercise trial, and a caloric restriction trial. During the exercise and no exercise trials, the participants consumed a standardized breakfast meal and $4 \mathrm{~h}$ later a standardized lunch. During the exercise trial the participants performed 45 min of cycling exercise at $\sim 53 \%$ of $\mathrm{VO}_{2}$ max beginning $45 \mathrm{~min}$ after the breakfast meal, while during the no exercise trial the participants remained sedentary the entire time. During the caloric restriction trial the caloric content of the breakfast meal was reduced to match the energy deficit of the exercise bout. Both exercise and caloric restriction reduced the postprandial glucose, insulin, and c-peptide response after the breakfast meal to a similar extent, but had no effect on the postprandial glucose, insulin, or c-peptide responses to the lunch meal. Larsen et al. [53] hypothesized that the reason why postprandial glucose levels were not reduced after lunch was because skeletal muscle glycogen levels were not depleted (although they did not measure this) during the moderate intensity exercise bout. Therefore, to determine if skeletal muscle glycogen depletion is required to reduce postprandial glucose after a lunch meal, Larsen et al. [54] performed another study to 
determine if high intensity interval exercise (known to deplete glycogen) would reduce postprandial glucose after breakfast and after lunch. The design of the study was similar to their first study [53], except high intensity interval exercise was performed instead of moderate intensity exercise. The researchers found that despite $27 \%$ lower vastus lateralis muscle glycogen levels after high intensity interval exercise, compared to no exercise, the exercise bout only reduced the postprandial glucose, insulin, and c-peptide response to the breakfast meal, but not the lunch meal. Thus, it appears that factors other than skeletal muscle glycogen depletion modulate the exercise induced reduction in postprandial glycemia, at least in the acute exercise bout model. Subsequent studies following these also showed that acute $\mathrm{AE}$ reduces postprandial glucose concentrations. For instance, Poirier et al. [46] demonstrated that $60 \mathrm{~min}$ of cycling at $\sim 60 \%$ of $\mathrm{VO}_{2}$ max reduced plasma glucose concentrations after a meal. In another study, walking for $20 \mathrm{~min}$ at a self-selected pace reduced the postprandial glucose response in patients with T2D [47].

The studies cited previously assessed the effect of exercise on postprandial glucose after one or two meals in a laboratory setting. Although these studies nicely demonstrated that exercise reduces postprandial glycemia, understanding the impact of exercise on $24 \mathrm{~h}$ blood glucose homeostasis outside of the lab in patients during free living conditions would be advantageous and have more practical significance for the "real world". The continuous glucose monitoring system (CGMS) is a sensitive tool that is inserted subcutaneously into the abdomen of users and continuously measures blood glucose every $5 \mathrm{~min}$. The CGMS assesses $24 \mathrm{~h}$ blood glucose outside of the lab during free-living conditions. The first study to utilize the CGMS to assess the effect of exercise on $24 \mathrm{~h}$ blood glucose profiles in patients with T2D was performed by Praet et al. [169]. 
In men with insulin treated $\mathrm{T} 2 \mathrm{D}, 45$ min of exercise (consisting of both $\mathrm{AE}$ and $\mathrm{RE}$ ) reduced the daily prevalence of hyperglycemia by $39 \%$ (hyperglycemia defined as blood glucose $>180 \mathrm{mg} / \mathrm{dL}$ ). In a subsequent study from the same lab, Manders et al. [170] assessed how AE intensity impacts $24 \mathrm{~h}$ blood glucose concentrations in patients with T2D. In males with non-insulin treated T2D, 60 min of low intensity cycling exercise (35\% of max W) significantly reduced the prevalence of daily hyperglycemia by $50 \%$. Although high intensity cycling exercise (30 min at $70 \%$ of $\max \mathrm{W}$ ) reduced the prevalence of daily hyperglycemia by $19 \%$, this decline was not significant. Similarly, 45 min of moderate intensity cycling (50\% of maximum workload capacity) reduced the prevalence of daily hyperglycemia by $33 \%$ in impaired glucose tolerant and non-insulin and insulin treated patients with T2D [50]. Another study showed similarly findings, such that a single bout of moderate-intensity cycling reduced daily hyperglycemia by an average of $31 \%$ in non-insulin and insulin treated patients with $\mathrm{T} 2 \mathrm{D}$, although the response was highly variable among patients [171].

It is clear that acute $\mathrm{AE}$ reduces post-meal glucose concentrations and improves $24 \mathrm{~h}$ glycemic control but the response is highly variable. It is not clear how many meals after an acute exercise bout that exercise induced reductions in post-meal glucose concentrations persist. Thus, to determine how a single exercise bout performed in the morning before breakfast alters post-meal glucose concentrations to each subsequent meal over the next $2 \mathrm{~d}$, Oberlin et al. [172] had patients with T2D perform $60 \mathrm{~min}$ of moderate intensity $\mathrm{AE}(60-75 \%$ of heart rate reserve) prior to breakfast, and measured the post-meal glucose response to 6 subsequent meals ( 3 each day). Exercise lowered the 24 $\mathrm{h}$ average blood glucose concentrations, compared to the no exercise trial, and only 
significantly lowered the post-meal glucose response to the lunch meal on day 1 . Thus, the researchers suggested that daily exercise may be most effective in improving postmeal glucose concentrations in type 2 diabetics.

Although daily exercise is often promoted, no study has assessed how daily vs. every other day exercise impacts postprandial hyperglycemia in patients with T2D. To address this question, van Dijk et al. [173] designed a study to assess $48 \mathrm{~h}$ blood glucose control in patients with T2D that performed either 30 min of cycling (50\% of max W) every day or $60 \mathrm{~min}$ of cycling every other day. The researchers reported that both exercise regimens reduced daily hyperglycemia by $\sim 8 \%$ over the $48 \mathrm{~h}$ glucose monitoring period.

Activities of daily living have also been shown to improve glycemic control in patients with T2D. Over a $24 \mathrm{~h}$ period, breaking up prolonged sitting with activities of daily living reduced the glucose area under the curve to breakfast, lunch, and dinner by $\sim 17 \%$ compared to no exercise [174]. However, one bout of moderate intensity exercise was much more effective, and reduced the glucose area under the curve to breakfast, lunch, and dinner by $\sim 35 \%$ compared to no exercise [174]. Thus, structured exercise is better at improving glycemic control than simply increasing activities of daily living, although benefits can be achieved from both strategies.

Lack of time is frequently cited as a barrier to exercise. Thus, high intensity interval training (HIT) has been gaining popularity because of its short duration, but the effect of HIT on $24 \mathrm{~h}$ glycemic control in patients with T2D has not been well established. Gillen et al. [175] assessed how HIT training (10 x $60 \mathrm{~s}$ cycling efforts at $\sim 90 \%$ of max HR) alters $24 \mathrm{~h}$ glycemic control in patients with T2D. These researchers 
reported that daily hyperglycemia was reduced by $\sim 10 \%$ and postprandial glucose excursions were reduced by $\sim 36 \%$ compared to no exercise.

\section{Blood Glucose Kinetics during Exercise}

In the fasted state, during $\mathrm{AE}$ glucose uptake in contracting skeletal muscle increases, but blood glucose concentrations remain relatively unchanged because of the simultaneous increase in hepatic glucose output that closely matches any increase in skeletal muscle glucose uptake in healthy non-diabetic patients [176-177]. On the contrary, in the fasted state in patients with T2D the increase in skeletal muscle glucose uptake during $\mathrm{AE}$ is not equally matched by an increase in hepatic glucose production, thus during AE blood glucose concentrations decrease [177]. It is not known if this same response occurs with $\mathrm{RE}$, an activity that does not require continuous muscle contractions.

\section{Acute Resistance Exercise and Glycemic Control in Type 2 Diabetes}

Aerobic exercise is often promoted to improve glycemic control in T2D, but RE has been gaining popularity as an alternative or complement to traditional AE. Only a few studies have examined the direct impact of acute RE on glycemic control or insulin sensitivity in patients with T2D. Fenicchia et al. [49] demonstrated that a single whole body RE session performed 12 to $24 \mathrm{~h}$ prior to an oral glucose tolerance test did not alter fasting glucose or insulin values but reduced the postprandial glucose response in patients with T2D. Similarly, Gordon et al. [68] showed no change in fasting blood glucose or insulin concentrations 24,48 , or $72 \mathrm{~h}$ after an acute RE session in patients with T2D. On the contrary, in pre-diabetic patients, acute RE lowered fasting blood glucose and insulin concentrations in a dose and intensity dependent manner [69]. These prior studies are 
limited as only a fasting blood glucose measure was taken or postprandial glucose was assessed after an oral glucose tolerance test, a common diagnostic test that is really not physiologically relevant. van Dijk et al. [50] was the first to examine how an acute bout of RE and AE impacts glycemic control with a mixed meal diet in patients with T2D. These researchers reported that $45 \mathrm{~min}$ of heavy RE (four exercises, three to five sets, 10 repetitions, $75 \%$ of 1-RM) significantly reduced $24 \mathrm{~h}$ average blood glucose concentrations. In addition, the acute RE bout reduced daily hyperglycemia by $35 \%$, an effect that was similar to $45 \mathrm{~min}$ of $\mathrm{AE}$ (cycling at $50 \%$ maximal power output).

However, not all studies suggest that acute RE improves glycemic control in patients with T2D. For instance, Fluckey et al. [38] demonstrated that an acute bout of whole body RE performed $\sim 18 \mathrm{~h}$ prior to a $75 \mathrm{~g}$ OGTT did not alter postprandial glucose concentrations but significantly reduced postprandial insulin concentrations in patients with T2D. Bacchi et al. [39] performed a study examining $48 \mathrm{~h}$ blood glucose profiles (assessed with a CGMS) in patients with T2D. After four months of either resistance training (three sessions per week, nine exercises, $10-12$ repetitions at $30-50 \%$ of $1-\mathrm{RM}$ ) or $\mathrm{AE}$ training (three sessions per week, $60 \mathrm{~min}$ per session, $60-65 \%$ of HRR), $48 \mathrm{~h}$ blood glucose profiles were measured with a CGMS in patients that performed a single, $60 \mathrm{~min}$ bout of exercise. During the $60 \mathrm{~min}$ acute exercise bout, blood glucose concentrations were significantly lower during AE, but not RE. Over the entire $48 \mathrm{~h}$ testing period, blood glucose concentrations were similar between no exercise, AE, and RE conditions. Given the mixed findings between studies that have examined the impact of an acute session of $\mathrm{RE}$ on glycemic control in patients with $\mathrm{T} 2 \mathrm{D}$, more research is warranted. 


\section{Exercise and Gastric Emptying}

Most studies examining how exercise alters gastric emptying were performed in healthy young patients and utilized a carbohydrate solution. These studies demonstrate that AE inhibits gastric emptying in an intensity dependent manner. For instance, Leiper et al. [66] demonstrated that high intensity cycling at $66 \%$ or $75 \%$ of $\mathrm{VO}_{2}$ max reduced gastric emptying by $\sim 15 \%$ and $\sim 36 \%$, respectively, compared to no exercise, in healthy men. Similarly, high intensity running slowed gastric emptying of a placebo solution and carbohydrate solution by $\sim 47 \%$ and $29 \%$, respectively, compared to low intensity walking in healthy men [67].

The impact that exercise has on gastric emptying in relation to postprandial glycemia has not been studied extensively. Gill et al. [178] demonstrated that 90 min of walking at $60 \% \mathrm{VO}_{2} \max$ the day prior to an oral fat tolerance test did not alter gastric emptying or postprandial glucose concentrations. Published observations from our lab show that 60 min of walking at $55-60 \%$ of $\mathrm{VO}_{2}$ peak the day prior to a meal did not alter postprandial glucose, GLP-1, or GIP concentrations in lean or obese patients [179]. The fact that GLP-1, GIP, or glucose was not different between trials in our study suggests that gastric emptying was unaltered (although gastric emptying was not directly measured). These two studies are limited by the fact that the exercise bout was performed the day prior to ingestion of a meal. The impact that exercise has on postprandial gastric emptying and how it relates to postprandial glycemia when the exercise bout is performed in closer proximity to a meal has been studied by only one investigator. Enevoldsen et al. [180] studied lean healthy men $(n=7)$ who performed 60 min of moderate intensity cycling beginning 90 min prior to a meal during one trial or 60 min 
after a meal during the other trial. It was reported that gastric emptying (assessed with acetaminophen) was similar between trials. However, this study has several limitations including 1) A no exercise trial was not used to compare with the two exercise trials, 2) the exercise bout was performed $60 \mathrm{~min}$ after the meal, $45 \mathrm{~min}$ after the acetaminophen peak in this study and at time when most of the meal has already been emptied from the stomach, 3) infrequent blood sampling thus the resolution of the change in the acetaminophen response was not clear, and 4) a small sample size was used.

Only one study has examined how AE alters gastric emptying in patients with T2D. In this study, long standing insulin dependent diabetics with diabetic gastroparesis (impaired gastric emptying) ingested a meal while gastric emptying was measured. The 30-min walking session started 90 min after ingestion of the meal. Gastric emptying was measured during the 90 min after ingestion of the meal and again 20 min after cessation of the walk. The researchers reported that walking increased gastric emptying in some patients (7/50), but had little effect on gastric emptying in most of the participants [181]. This study was limited by the fact that gastric emptying was measured after the meal had been emptied from the stomach and after the walking session. It is not known how gastric emptying of a mixed meal is altered when RE is performed either immediately before or soon after a mixed meal in patients with T2D.

\section{Exercise and Post-meal Incretin Hormone Responses}

\section{Acute Exercise}

The majority of research examining the impact of acute exercise on postprandial incretin hormone responses has utilized a non-diabetic population. Previous observations from our lab indicate that $1 \mathrm{~h}$ of moderate intensity AE the night prior to a mixed meal in 
the morning reduced the postprandial insulin and TAG response in obese patients, and these changes occurred without changes in postprandial GLP-1 and GIP responses [182]. Using a similar study design, Dekker et al. [183] reported that $1 \mathrm{~h}$ of moderate intensity $\mathrm{AE}$ in hypertriacylglycerolemic obese men the day before a morning oral fat tolerance test did not alter postprandial GIP or GLP-1 concentrations. These studies suggest that when exercise is performed the day prior to a mixed meal postprandial incretin hormone concentrations are not altered.

On the contrary, other studies have shown that acute AE performed immediately prior to or after ingestion of a meal can alter postprandial incretin hormone responses. For instance, in trained athletes that performed $2 \mathrm{~h}$ of moderate intensity AE immediately prior to oral glucose ingestion, the postprandial insulin and GIP response, but not the GLP-1 response, was attenuated [184]. Similarly, in trained male athletes prior exhaustive AE reduced postprandial GIP responses after glucose ingestion [185]. These studies suggest that prolonged $\mathrm{AE}$ prior to ingestion of an oral glucose tolerance test reduces postprandial GIP responses. However, when the timing of the exercise bout is moved to after meal ingestion, research has shown that moderate or high intensity $\mathrm{AE}$ augments the postprandial GLP-1 response in non-diabetic patients [62, 186-187]. Thus, the timing of exercise around a meal has an impact on the postprandial incretin hormone response in healthy patients.

The effect of acute $\mathrm{AE}$ on postprandial incretin hormone responses in patients with T2D has only been assessed by one investigator. After 28 days of metformin or placebo treatment, AE performed prior to a lunch meal did not alter postprandial GLP-1 
or GIP responses [86]. However, metformin did increase preprandial GLP-1 and GIP and postprandial GLP-1 concentrations.

\section{Exercise Training}

Three cross sectional studies have compared postprandial GIP and GLP-1 responses in trained vs. untrained subjects, and the responses seem to depend on the type of test meal given. In the study by Lund et al. [188], after ingestion of a mixed meal shake postprandial GIP responses were lower in aerobically trained compared to untrained patients, while postprandial GLP-1 responses were similar between the groups. However, fasting GLP-1 concentrations were higher in aerobically trained patients. Interestingly, these same researchers demonstrated in another study employing an oral glucose tolerance test that insulin secretion, but not postprandial GLP-1 or GIP concentrations, were lower in trained vs. untrained patients. Similarly, Weiss et al. [189] reported that postprandial insulin responses, but not GIP or GLP-1 responses, were lower in aerobically trained vs. untrained patients after consumption of a $75 \mathrm{~g}$ oral glucose tolerance test. Taken together, postprandial GIP responses are lower in trained vs. untrained patients after a mixed meal, while postprandial GIP responses after an oral glucose tolerance test are not different between trained and untrained non-diabetic patients.

Only one study has assessed how exercise training impacts postprandial GIP responses in type 2 diabetics. After exercise and diet-induced weight loss $(\sim 5.0 \mathrm{~kg})$, the postprandial GIP response was higher in obese type 2 diabetic subjects, and this was associated with increased insulin secretion and improved beta-cell function [190]. 


\section{Exercise and Beta-cell Function}

Acute or Short Term Aerobic Exercise and Beta-cell Function

To date, only one study has examined how short-term AE alters beta-cell function. The first study to do so reported that $7 \mathrm{~d}$ of moderate intensity AE $(1 \mathrm{~h} / \mathrm{d})$ increased the disposition index (measure of beta cell function) by $31 \%$ in older patients with impaired glucose tolerance [191]. No study has assessed how acute AE or RE alters beta-cell function in patients with T2D.

Exercise Training and Beta Cell Function

The majority of studies show that AE training improves beta-cell function in nondiabetic and diabetic adults. For instance, Slentz et al. [192] reported that an 8-month exercise program with either moderate or vigorous exercise intensities improved the disposition index (measure of beta-cell function) in overweight adults, with moderate intensity exercise having the biggest impact. Similarly, Malin et al. [193] reported that an exercise training and weight loss intervention improved the disposition index in older patients with pre-diabetes. Further, the increase in the disposition index due to exercise training occurred in a linear dose-response manner such that higher exercise doses (> 2,000 kcal/wk) improved beta-cell function more so than lower exercise doses. This same group also showed that an exercise and diet-induced weight loss program in patients with T2D improved beta-cell function, and that this was associated with increased glucose stimulated GIP concentrations [190]. Interesting, diet composition influences the effect of an exercise-training intervention on beta-cell function. For example, Solomon et al. [194] reported that a low-GI diet did not alter beta-cell function after an exercise and diet induced weight-loss intervention, whereas a high-GI diet impaired beta-cell function 
during a similar intervention, despite similar reductions in weight and improvements in insulin sensitivity occurred during both dietary interventions. Resistance training has also been shown to improve beta-cell function. Croymans et al. [87] demonstrated that a 12wk resistance training program ( $3 \mathrm{RE}$ sessions per wk) improved beta-cell function in overweight/obese, sedentary young men.

Not all studies suggest that AE or RE improves beta-cell function. For instance, resistance training performed twice weekly for 16-wks did not alter beta-cell function in overweight adolescent Latinos [88]. Similarly, Bacchi et al. [89] demonstrated four months of AE or RE did not alter beta-cell function in patients with T2D. 


\section{REFERENCES}

1. Diagnosis and classification of diabetes mellitus. Diabetes Care, 2014. 37 Suppl 1: p. S81-90.

2. Mokdad, A.H., et al., Diabetes trends in the U.S.: 1990-1998. Diabetes Care, 2000. 23(9): p. 1278-83.

3. Mokdad, A.H., et al., Prevalence of obesity, diabetes, and obesity-related health risk factors, 2001. JAMA, 2003. 289(1): p. 76-9.

4. Satman, I., et al., Twelve-year trends in the prevalence and risk factors of diabetes and prediabetes in Turkish adults. Eur J Epidemiol, 2013.

5. Danaei, G., et al., National, regional, and global trends in fasting plasma glucose and diabetes prevalence since 1980: systematic analysis of health examination surveys and epidemiological studies with 370 country-years and 2.7 million participants. Lancet, 2011. 378(9785): p. 31-40.

6. Number (in Millions) of Civilian, Noninstitutionalized Persons with Diagnosed Diabetes, United States, 1960-2010. 2011.

7. World Health Organization. The top 10 causes of death. Retrieved May 2014 from http://www.who.int/mediacentre/factsheets/fs310/en/.

8. Centers for Disease Control and Prevention. 2011 National diabetes fact sheet. Retreived May 2014 from http://www.cdc.gov/diabetes/pubs/factsheet11.htm.

9. Brandle, M., et al., The direct medical cost of type 2 diabetes. Diabetes Care, 2003. 26(8): p. 2300-4.

10. O'Brien, J.A., A.R. Patrick, and J.J. Caro, Cost of managing complications resulting from type 2 diabetes mellitus in Canada. BMC Health Serv Res, 2003. 3(1): p. 7.

11. Gandra, S.R., et al., Total and component health care costs in a non-Medicare HMO population of patients with and without type 2 diabetes and with and without macrovascular disease. J Manag Care Pharm, 2006. 12(7): p. 546-54.

12. Wang, W., et al., Type 2 diabetes mellitus in China: a preventable economic burden. Am J Manag Care, 2009. 15(9): p. 593-601.

13. Church, T.S., et al., Effects of aerobic and resistance training on hemoglobin A1c levels in patients with type 2 diabetes: a randomized controlled trial. JAMA, 2010. 304(20): p. 2253-62.

14. Sigal, R.J., et al., Effects of aerobic training, resistance training, or both on glycemic control in type 2 diabetes: a randomized trial. Ann Intern Med, 2007. 147(6): p. 357-69.

15. Agurs-Collins, T.D., et al., A randomized controlled trial of weight reduction and exercise for diabetes management in older African-American subjects. Diabetes Care, 1997. 20(10): p. 1503-11.

16. Dunstan, D.W., et al., The independent and combined effects of aerobic exercise and dietary fish intake on serum lipids and glycemic control in NIDDM. A randomized controlled study. Diabetes Care, 1997. 20(6): p. 913-21.

17. Raz, I., E. Hauser, and M. Bursztyn, Moderate exercise improves glucose metabolism in uncontrolled elderly patients with non-insulin-dependent diabetes mellitus. Isr J Med Sci, 1994. 30(10): p. 766-70. 
18. Ronnemaa, T., et al., A controlled randomized study on the effect of long-term physical exercise on the metabolic control in type 2 diabetic patients. Acta Med Scand, 1986. 220(3): p. 219-24.

19. Dunstan, D.W., et al., Effects of a short-term circuit weight training program on glycaemic control in NIDDM. Diabetes Res Clin Pract, 1998. 40(1): p. 53-61.

20. Honkola, A., T. Forsen, and J. Eriksson, Resistance training improves the metabolic profile in individuals with type 2 diabetes. Acta Diabetol, 1997. 34(4): p. 245-8.

21. Lehmann, R., et al., Loss of abdominal fat and improvement of the cardiovascular risk profile by regular moderate exercise training in patients with NIDDM. Diabetologia, 1995. 38(11): p. 1313-9.

22. Vanninen, E., et al., Habitual physical activity, aerobic capacity and metabolic control in patients with newly-diagnosed type 2 (non-insulin-dependent) diabetes mellitus: effect of 1-year diet and exercise intervention. Diabetologia, 1992. 35(4): p. 340-6.

23. Wing, R.R., et al., Exercise in a behavioural weight control programme for obese patients with Type 2 (non-insulin-dependent) diabetes. Diabetologia, 1988. 31(12): p. 902-9.

24. Giannopoulou, I., et al., Exercise is required for visceral fat loss in postmenopausal women with type 2 diabetes. J Clin Endocrinol Metab, 2005. 90(3): p. 1511-8.

25. Tessier, D., et al., Effects of aerobic physical exercise in the elderly with type 2 diabetes mellitus. Arch Gerontol Geriatr, 2000. 31(2): p. 121-132.

26. Mourier, A., et al., Mobilization of visceral adipose tissue related to the improvement in insulin sensitivity in response to physical training in NIDDM. Effects of branched-chain amino acid supplements. Diabetes Care, 1997. 20(3): p. 385-91.

27. Boudou, P., et al., Effects of a single bout of exercise and exercise training on steroid levels in middle-aged type 2 diabetic men: relationship to abdominal adipose tissue distribution and metabolic status. Diabetes Metab, 2000. 26(6): p. 450-7.

28. Baldi, J.C. and N. Snowling, Resistance training improves glycaemic control in obese type 2 diabetic men. Int J Sports Med, 2003. 24(6): p. 419-23.

29. Cuff, D.J., et al., Effective exercise modality to reduce insulin resistance in women with type 2 diabetes. Diabetes Care, 2003. 26(11): p. 2977-82.

30. Castaneda, C., et al., A randomized controlled trial of resistance exercise training to improve glycemic control in older adults with type 2 diabetes. Diabetes Care, 2002. 25(12): p. 2335-41.

31. Loimaala, A., et al., Exercise training improves baroreflex sensitivity in type 2 diabetes. Diabetes, 2003. 52(7): p. 1837-42.

32. Colberg, S.R., et al., Exercise and type 2 diabetes: the American College of Sports Medicine and the American Diabetes Association: joint position statement executive summary. Diabetes Care, 2010. 33(12): p. 2692-6.

33. Colberg, S.R., et al., Exercise and type 2 diabetes: American College of Sports Medicine and the American Diabetes Association: joint position statement. Exercise and type 2 diabetes. Med Sci Sports Exerc, 2010. 42(12): p. 2282-303. 
34. Colberg, S.R., et al., Exercise and type 2 diabetes: the American College of Sports Medicine and the American Diabetes Association: joint position statement. Diabetes Care, 2010. 33(12): p. e147-67.

35. Boule, N.G., et al., Effects of exercise training on glucose homeostasis: the HERITAGE Family Study. Diabetes Care, 2005. 28(1): p. 108-14.

36. Van Dijk, J.W., et al., Exercise and 24-h glycemic control: equal effects for all type 2 diabetes patients? Med Sci Sports Exerc, 2013. 45(4): p. 628-35.

37. Gill, J.M., et al., Effect of prior moderate exercise on postprandial metabolism in men with type 2 diabetes: heterogeneity of responses. Atherosclerosis, 2007. 194(1): p. 134-43.

38. Fluckey, J.D., et al., Effects of resistance exercise on glucose tolerance in normal and glucose-intolerant subjects. J Appl Physiol (1985), 1994. 77(3): p. 1087-92.

39. Bacchi, E., et al., Differences in the acute effects of aerobic and resistance exercise in subjects with type 2 diabetes: results from the RAED2 Randomized Trial. PLoS One, 2012. 7(12): p. e49937.

40. Dalgaard, M., C. Thomsen, and K. Hermansen, Effects of one single bout of lowintensity exercise on postprandial lipaemia in type 2 diabetic men. Br J Nutr, 2004. 92(3): p. 469-76.

41. Ruchat, S.M., et al., Improvements in glucose homeostasis in response to regular exercise are influenced by the PPARG Pro12Ala variant: results from the HERITAGE Family Study. Diabetologia, 2010. 53(4): p. 679-89.

42. Malin, S.K. and J.P. Kirwan, Fasting hyperglycaemia blunts the reversal of impaired glucose tolerance after exercise training in obese older adults. Diabetes Obes Metab, 2012. 14(9): p. 835-41.

43. Solomon, T.P., et al., The influence of hyperglycemia on the therapeutic effect of exercise on glycemic control in patients with type 2 diabetes mellitus. JAMA Intern Med, 2013. 173(19): p. 1834-6.

44. Gaudet-Savard, T., et al., Safety and magnitude of changes in blood glucose levels following exercise performed in the fasted and the postprandial state in men with type 2 diabetes. Eur J Cardiovasc Prev Rehabil, 2007. 14(6): p. 831-6.

45. Poirier, P., et al., Impact of time interval from the last meal on glucose response to exercise in subjects with type 2 diabetes. J Clin Endocrinol Metab, 2000. 85(8): p. 2860-4.

46. Poirier, P., et al., Prior meal enhances the plasma glucose lowering effect of exercise in type 2 diabetes. Med Sci Sports Exerc, 2001. 33(8): p. 1259-64.

47. Colberg, S.R., et al., Postprandial walking is better for lowering the glycemic effect of dinner than pre-dinner exercise in type 2 diabetic individuals. J Am Med Dir Assoc, 2009. 10(6): p. 394-7.

48. Tobin, L.W., B. Kiens, and H. Galbo, The effect of exercise on postprandial lipidemia in type 2 diabetic patients. Eur J Appl Physiol, 2008. 102(3): p. 361-70.

49. Fenicchia, L.M., et al., Influence of resistance exercise training on glucose control in women with type 2 diabetes. Metabolism, 2004. 53(3): p. 284-9.

50. van Dijk, J.W., et al., Both resistance- and endurance-type exercise reduce the prevalence of hyperglycaemia in individuals with impaired glucose tolerance and in insulin-treated and non-insulin-treated type 2 diabetic patients. Diabetologia, 2012. 55(5): p. 1273-82. 
51. Garg, A., et al., Effects of varying carbohydrate content of diet in patients with noninsulin-dependent diabetes mellitus. JAMA, 1994. 271(18): p. 1421-8.

52. Abbasi, F., et al., Effect of metformin treatment on multiple cardiovascular disease risk factors in patients with type 2 diabetes mellitus. Metabolism, 2004. 53(2): p. 159-64.

53. Larsen, J.J., et al., The effect of moderate exercise on postprandial glucose homeostasis in NIDDM patients. Diabetologia, 1997. 40(4): p. 447-53.

54. Larsen, J.J., et al., The effect of intense exercise on postprandial glucose homeostasis in type II diabetic patients. Diabetologia, 1999. 42(11): p. 1282-92.

55. Singhal, P., et al., Regulation of endogenous glucose production after a mixed meal in type 2 diabetes. Am J Physiol Endocrinol Metab, 2002. 283(2): p. E275-83.

56. Laakso, M., et al., Impaired insulin-mediated skeletal muscle blood flow in patients with NIDDM. Diabetes, 1992. 41(9): p. 1076-83.

57. Thorell, A., et al., Exercise and insulin cause GLUT-4 translocation in human skeletal muscle. Am J Physiol, 1999. 277(4 Pt 1): p. E733-41.

58. Cline, G.W., et al., Impaired glucose transport as a cause of decreased insulinstimulated muscle glycogen synthesis in type 2 diabetes. N Engl J Med, 1999. 341(4): p. 240-6.

59. Kennedy, J.W., et al., Acute exercise induces GLUT4 translocation in skeletal muscle of normal human subjects and subjects with type 2 diabetes. Diabetes, 1999. 48(5): p. 1192-7.

60. Henriksen, E.J., et al., Glucose transporter protein content and glucose transport capacity in rat skeletal muscles. Am J Physiol, 1990. 259(4 Pt 1): p. E593-8.

61. Ploug, T., et al., Kinetics of glucose transport in rat skeletal muscle membrane vesicles: effects of insulin and contractions. Am J Physiol, 1992. 262(5 Pt 1): p. E700-11.

62. Martins, C., et al., Effects of exercise on gut peptides, energy intake and appetite. J Endocrinol, 2007. 193(2): p. 251-8.

63. Nauck, M.A., et al., Glucagon-like peptide 1 inhibition of gastric emptying outweighs its insulinotropic effects in healthy humans. Am J Physiol, 1997. 273(5 Pt 1): p. E981-8.

64. Willms, B., et al., Gastric emptying, glucose responses, and insulin secretion after a liquid test meal: effects of exogenous glucagon-like peptide-1 (GLP-1)-(7-36) amide in type 2 (noninsulin-dependent) diabetic patients. J Clin Endocrinol Metab, 1996. 81(1): p. 327-32.

65. Wettergren, A., et al., Truncated GLP-1 (proglucagon 78-107-amide) inhibits gastric and pancreatic functions in man. Dig Dis Sci, 1993. 38(4): p. 665-73.

66. Leiper, J.B., N.P. Broad, and R.J. Maughan, Effect of intermittent high-intensity exercise on gastric emptying in man. Med Sci Sports Exerc, 2001. 33(8): p. 1270-8.

67. Leiper, J.B., et al., The effect of intermittent high-intensity running on gastric emptying of fluids in man. Med Sci Sports Exerc, 2005. 37(2): p. 240-7.

68. Gordon, B.A., et al., Insulin sensitivity not modulated 24 to $78 \mathrm{~h}$ after acute resistance exercise in type 2 diabetes patients. Diabetes Obes Metab, 2013. 15(5): p. $478-80$. 
69. Black, L.E., P.D. Swan, and B.A. Alvar, Effects of intensity and volume on insulin sensitivity during acute bouts of resistance training. J Strength Cond Res, 2010. 24(4): p. 1109-16.

70. Heading, R.C., et al., The dependence of paracetamol absorption on the rate of gastric emptying. Br J Pharmacol, 1973. 47(2): p. 415-21.

71. Bijlani, R.L., et al., Effect of coingestion of paracetamol on glycaemic response. Indian J Physiol Pharmacol, 1992. 36(3): p. 215-8.

72. Karpe, F. and A. Hamsten, Determination of apolipoproteins B-48 and B-100 in triglyceride-rich lipoproteins by analytical SDS-PAGE. J Lipid Res, 1994. 35(7): p. 1311-7.

73. Mari, A., et al., Meal and oral glucose tests for assessment of beta -cell function: modeling analysis in normal subjects. Am J Physiol Endocrinol Metab, 2002. 283(6): p. E1159-66.

74. Mari, A., et al., Assessing insulin secretion by modeling in multiple-meal tests: role of potentiation. Diabetes, 2002. 51 Suppl 1: p. S221-6.

75. Van Cauter, E., et al., Estimation of insulin secretion rates from $C$-peptide levels. Comparison of individual and standard kinetic parameters for C-peptide clearance. Diabetes, 1992. 41(3): p. 368-77.

76. Pruessner, J.C., et al., Two formulas for computation of the area under the curve represent measures of total hormone concentration versus time-dependent change. Psychoneuroendocrinology, 2003. 28(7): p. 916-31.

77. Schlichtkrull, J., O. Munck, and M. Jersild, The M-Valve, an Index of Blood-Sugar Control in Diabetics. Acta Med Scand, 1965. 177: p. 95-102.

78. Service, F.J., et al., Mean amplitude of glycemic excursions, a measure of diabetic instability. Diabetes, 1970. 19(9): p. 644-55.

79. Wojcicki, J.M., "J"-index. A new proposition of the assessment of current glucose control in diabetic patients. Horm Metab Res, 1995. 27(1): p. 41-2.

80. McDonnell, C.M., et al., A novel approach to continuous glucose analysis utilizing glycemic variation. Diabetes Technol Ther, 2005. 7(2): p. 253-63.

81. Ryan, E.A. and A.J. Shapiro, A patient with severe, recurrent hypoglycemia and glycemic lability who underwent islet transplantation. Nat Clin Pract Endocrinol Metab, 2006. 2(6): p. 349-53; quiz 354.

82. Bouchard, C., et al., Adverse metabolic response to regular exercise: is it a rare or common occurrence? PLoS One, 2012. 7(5): p. e37887.

83. Phillips, B., et al., Resistance exercise training improves age-related declines in leg vascular conductance and rejuvenates acute leg blood flow responses to feeding and exercise. J Appl Physiol (1985), 2012. 112(3): p. 347-53.

84. Ginsberg, H.N., Y.L. Zhang, and A. Hernandez-Ono, Regulation of plasma triglycerides in insulin resistance and diabetes. Arch Med Res, 2005. 36(3): p. 23240.

85. Hennes, M.M., A. Dua, and A.H. Kissebah, Effects of free fatty acids and glucose on splanchnic insulin dynamics. Diabetes, 1997. 46(1): p. 57-62.

86. Eshghi, S.R., G.J. Bell, and N.G. Boule, Effects of Aerobic Exercise with or without Metformin on Plasma Incretins in Type 2 Diabetes. Can J Diabetes, 2013. 37(6): p. 375-80. 
87. Croymans, D.M., et al., Resistance training improves indices of muscle insulin sensitivity and beta-cell function in overweight/obese, sedentary young men. J Appl Physiol (1985), 2013. 115(9): p. 1245-53.

88. Shaibi, G.Q., et al., Effects of resistance training on insulin sensitivity in overweight Latino adolescent males. Med Sci Sports Exerc, 2006. 38(7): p. 1208-15.

89. Bacchi, E., et al., Metabolic effects of aerobic training and resistance training in type 2 diabetic subjects: a randomized controlled trial (the RAED2 study). Diabetes Care, 2012. 35(4): p. 676-82.

90. Naslund, E., et al., Gastric emptying: comparison of scintigraphic, polyethylene glycol dilution, and paracetamol tracer assessment techniques. Scand $\mathrm{J}$ Gastroenterol, 2000. 35(4): p. 375-9.

91. Roy, D., M. Perreault, and A. Marette, Insulin stimulation of glucose uptake in skeletal muscles and adipose tissues in vivo is NO dependent. Am J Physiol, 1998. 274(4 Pt 1): p. E692-9.

92. Sindelar, D.K., et al., A comparison of the effects of selective increases in peripheral or portal insulin on hepatic glucose production in the conscious dog. Diabetes, 1996. 45(11): p. 1594-604.

93. Sadur, C.N. and R.H. Eckel, Insulin stimulation of adipose tissue lipoprotein lipase. Use of the euglycemic clamp technique. J Clin Invest, 1982. 69(5): p. 1119-25.

94. Cherrington, A.D., et al., Control of hepatic glucose output by glucagon and insulin in the intact dog. Biochem Soc Symp, 1978(43): p. 31-45.

95. Christensen, M., et al., Glucose-dependent insulinotropic polypeptide: a bifunctional glucose-dependent regulator of glucagon and insulin secretion in humans. Diabetes, 2011. 60(12): p. 3103-9.

96. Kreymann, B., et al., Glucagon-like peptide-1 7-36: a physiological incretin in man. Lancet, 1987. 2(8571): p. 1300-4.

97. Schwizer, W., et al., Role of cholecystokinin in the regulation of liquid gastric emptying and gastric motility in humans: studies with the CCK antagonist loxiglumide. Gut, 1997. 41(4): p. 500-4.

98. Pilichiewicz, A.N., et al., Load-dependent effects of duodenal glucose on glycemia, gastrointestinal hormones, antropyloroduodenal motility, and energy intake in healthy men. Am J Physiol Endocrinol Metab, 2007. 293(3): p. E743-53.

99. Ma, J., et al., Effects of variations in duodenal glucose load on glycaemic, insulin, and incretin responses in type 2 diabetes. Diabet Med, 2012. 29(5): p. 604-8.

100. Gonlachanvit, S., et al., Effect of altering gastric emptying on postprandial plasma glucose concentrations following a physiologic meal in type-II diabetic patients. Dig Dis Sci, 2003. 48(3): p. 488-97.

101. Meier, J.J., et al., Glucagon-like peptide 1 abolishes the postprandial rise in triglyceride concentrations and lowers levels of non-esterified fatty acids in humans. Diabetologia, 2006. 49(3): p. 452-8.

102. Unger, R.H. and L. Orci, The essential role of glucagon in the pathogenesis of diabetes mellitus. Lancet, 1975. 1(7897): p. 14-6.

103. Bell, P.M., R.G. Firth, and R.A. Rizza, Assessment of the postprandial pattern of glucose metabolism in nondiabetic subjects and patients with non-insulindependent diabetes mellitus using a simultaneous infusion of [2(3)H] and [3(3)H] glucose. Metabolism, 1989. 38(1): p. 38-45. 
104. Ferrannini, E., et al., The disposal of an oral glucose load in patients with noninsulin-dependent diabetes. Metabolism, 1988. 37(1): p. 79-85.

105. Firth, R.G., et al., Postprandial hyperglycemia in patients with noninsulindependent diabetes mellitus. Role of hepatic and extrahepatic tissues. J Clin Invest, 1986. 77(5): p. 1525-32.

106. Kelley, D., M. Mokan, and T. Veneman, Impaired postprandial glucose utilization in non-insulin-dependent diabetes mellitus. Metabolism, 1994. 43(12): p. 1549-57.

107. Mitrakou, A., et al., Contribution of abnormal muscle and liver glucose metabolism to postprandial hyperglycemia in NIDDM. Diabetes, 1990. 39(11): p. 1381-90.

108. Woerle, H.J., et al., Mechanisms for abnormal postprandial glucose metabolism in type 2 diabetes. Am J Physiol Endocrinol Metab, 2006. 290(1): p. E67-E77.

109. Petersen, K.F., et al., Reversal of nonalcoholic hepatic steatosis, hepatic insulin resistance, and hyperglycemia by moderate weight reduction in patients with type 2 diabetes. Diabetes, 2005. 54(3): p. 603-8.

110. Basu, R., et al., Pathogenesis of prediabetes: role of the liver in isolated fasting hyperglycemia and combined fasting and postprandial hyperglycemia. J Clin Endocrinol Metab, 2013. 98(3): p. E409-17.

111. Shah, P., et al., Lack of suppression of glucagon contributes to postprandial hyperglycemia in subjects with type 2 diabetes mellitus. J Clin Endocrinol Metab, 2000. 85(11): p. 4053-9.

112. Rizza, R.A., Pathogenesis of fasting and postprandial hyperglycemia in type 2 diabetes: implications for therapy. Diabetes, 2010. 59(11): p. 2697-707.

113. Rask, E., et al., Impaired incretin response after a mixed meal is associated with insulin resistance in nondiabetic men. Diabetes Care, 2001. 24(9): p. 1640-5.

114. Vilsboll, T., et al., Incretin secretion in relation to meal size and body weight in healthy subjects and people with type 1 and type 2 diabetes mellitus. J Clin Endocrinol Metab, 2003. 88(6): p. 2706-13.

115. Vilsboll, T., et al., Reduced postprandial concentrations of intact biologically active glucagon-like peptide 1 in type 2 diabetic patients. Diabetes, 2001. 50(3): p. 60913.

116. Vilsboll, T., et al., Defective amplification of the late phase insulin response to glucose by GIP in obese Type II diabetic patients. Diabetologia, 2002. 45(8): p. 1111-9.

117. Schwartz, J.G., et al., Rapid gastric emptying of a solid pancake meal in type II diabetic patients. Diabetes Care, 1996. 19(5): p. 468-71.

118. Bertin, E., et al., Gastric emptying is accelerated in obese type 2 diabetic patients without autonomic neuropathy. Diabetes Metab, 2001. 27(3): p. 357-64.

119. Lipp, R.W., et al., Evidence of accelerated gastric emptying in longstanding diabetic patients after ingestion of a semisolid meal. J Nucl Med, 1997. 38(5): p. 814-8.

120. Kannel, W.B. and D.L. McGee, Diabetes and cardiovascular disease. The Framingham study. JAMA, 1979. 241(19): p. 2035-8.

121. Sharma, M.D., J.A. Farmer, and A. Garber, Type 2 diabetes and cardiovascular risk factors. Curr Med Res Opin, 2011. 27 Suppl 3: p. 1-5.

122. Laing, S.P., et al., Mortality from heart disease in a cohort of 23,000 patients with insulin-treated diabetes. Diabetologia, 2003. 46(6): p. 760-5. 
123. Dorman, J.S., et al., The Pittsburgh insulin-dependent diabetes mellitus (IDDM) morbidity and mortality study. Mortality results. Diabetes, 1984. 33(3): p. 271-6.

124. Hanefeld, M., et al., Postprandial plasma glucose is an independent risk factor for increased carotid intima-media thickness in non-diabetic individuals. Atherosclerosis, 1999. 144(1): p. 229-35.

125. Zhang, Y.F., et al., Hyperglycaemia after glucose loading is a major predictor of preclinical atherosclerosis in nondiabetic subjects. Clin Endocrinol (Oxf), 2006. 64(2): p. 153-7.

126. Temelkova-Kurktschiev, T.S., et al., Increased intimal-medial thickness in newly detected type 2 diabetes: risk factors. Diabetes Care, 1999. 22(2): p. 333-8.

127. Hanefeld, M., et al., Post-challenge hyperglycaemia relates more strongly than fasting hyperglycaemia with carotid intima-media thickness: the RIAD Study. Risk Factors in Impaired Glucose Tolerance for Atherosclerosis and Diabetes. Diabet Med, 2000. 17(12): p. 835-40.

128. Hu, Y., et al., Postchallenge plasma glucose excursions, carotid intima-media thickness, and risk factors for atherosclerosis in Chinese population with type 2 diabetes. Atherosclerosis, 2010. 210(1): p. 302-6.

129. Kim, H.J., et al., The level of 2-h post-challenge glucose is an independent risk factor of carotid intima-media thickness progression in Korean type 2 diabetic patients. J Diabetes Complications, 2007. 21(1): p. 7-12.

130. Temelkova-Kurktschiev, T.S., et al., Postchallenge plasma glucose and glycemic spikes are more strongly associated with atherosclerosis than fasting glucose or HbA1c level. Diabetes Care, 2000. 23(12): p. 1830-4.

131. Versari, D., et al., Endothelial dysfunction as a target for prevention of cardiovascular disease. Diabetes Care, 2009. 32 Suppl 2: p. S314-21.

132. Tesfamariam, B., et al., Elevated glucose promotes generation of endotheliumderived vasoconstrictor prostanoids in rabbit aorta. J Clin Invest, 1990. 85(3): p. 929-32.

133. Durante, W., A.K. Sen, and F.A. Sunahara, Impairment of endothelium-dependent relaxation in aortae from spontaneously diabetic rats. Br J Pharmacol, 1988. 94(2): p. 463-8.

134. Meraji, S., et al., Endothelium-dependent relaxation in aorta of BB rat. Diabetes, 1987. 36(8): p. 978-81.

135. Makimattila, S., et al., Chronic hyperglycemia impairs endothelial function and insulin sensitivity via different mechanisms in insulin-dependent diabetes mellitus. Circulation, 1996. 94(6): p. 1276-82.

136. Johnstone, M.T., et al., Impaired endothelium-dependent vasodilation in patients with insulin-dependent diabetes mellitus. Circulation, 1993. 88(6): p. 2510-6.

137. Gupta, S., et al., Endothelium-dependent inhibition of $\mathrm{Na}(+)-\mathrm{K}+$ ATPase activity in rabbit aorta by hyperglycemia. Possible role of endothelium-derived nitric oxide. $\mathrm{J}$ Clin Invest, 1992. 90(3): p. 727-32.

138. Ding, Y., et al., Effects of simulated hyperglycemia, insulin, and glucagon on endothelial nitric oxide synthase expression. Am J Physiol Endocrinol Metab, 2000. 279(1): p. E11-7.

139. Noyman, I., et al., Hyperglycemia reduces nitric oxide synthase and glycogen synthase activity in endothelial cells. Nitric Oxide, 2002. 7(3): p. 187-93. 
140. Du, X.L., et al., Hyperglycemia inhibits endothelial nitric oxide synthase activity by posttranslational modification at the Akt site. J Clin Invest, 2001. 108(9): p. 1341-8.

141. Tesfamariam, B. and R.A. Cohen, Free radicals mediate endothelial cell dysfunction caused by elevated glucose. Am J Physiol, 1992. 263(2 Pt 2): p. H3216.

142. Marfella, R., et al., Glutathione reverses systemic hemodynamic changes induced by acute hyperglycemia in healthy subjects. Am J Physiol, 1995. 268(6 Pt 1): p. E1167-73.

143. Ting, H.H., et al., Vitamin C improves endothelium-dependent vasodilation in patients with non-insulin-dependent diabetes mellitus. J Clin Invest, 1996. 97(1): p. 22-8.

144. Brownlee, M., A. Cerami, and H. Vlassara, Advanced glycosylation end products in tissue and the biochemical basis of diabetic complications. N Engl J Med, 1988. 318(20): p. 1315-21.

145. Bucala, R., et al., Identification of the major site of apolipoprotein B modification by advanced glycosylation end products blocking uptake by the low density lipoprotein receptor. J Biol Chem, 1995. 270(18): p. 10828-32.

146. Bucala, R., et al., Lipid advanced glycosylation: pathway for lipid oxidation in vivo. Proc Natl Acad Sci U S A, 1993. 90(14): p. 6434-8.

147. Yan, S.D., et al., Enhanced cellular oxidant stress by the interaction of advanced glycation end products with their receptors/binding proteins. J Biol Chem, 1994. 269(13): p. 9889-97.

148. Wautier, J.L., et al., Advanced glycation end products (AGEs) on the surface of diabetic erythrocytes bind to the vessel wall via a specific receptor inducing oxidant stress in the vasculature: a link between surface-associated AGEs and diabetic complications. Proc Natl Acad Sci U S A, 1994. 91(16): p. 7742-6.

149. Wautier, J.L., et al., Receptor-mediated endothelial cell dysfunction in diabetic vasculopathy. Soluble receptor for advanced glycation end products blocks hyperpermeability in diabetic rats. J Clin Invest, 1996. 97(1): p. 238-43.

150. Park, L., et al., Suppression of accelerated diabetic atherosclerosis by the soluble receptor for advanced glycation endproducts. Nat Med, 1998. 4(9): p. 1025-31.

151. Nilsson-Berglund, L.M., et al., Nuclear factor of activated $T$ cells regulates osteopontin expression in arterial smooth muscle in response to diabetes-induced hyperglycemia. Arterioscler Thromb Vasc Biol, 2010. 30(2): p. 218-24.

152. Ros, E., Intestinal absorption of triglyceride and cholesterol. Dietary and pharmacological inhibition to reduce cardiovascular risk. Atherosclerosis, 2000. 151(2): p. 357-79.

153. Packard, C.J. and J. Shepherd, Lipoprotein heterogeneity and apolipoprotein B metabolism. Arterioscler Thromb Vasc Biol, 1997. 17(12): p. 3542-56.

154. Bjorkegren, J., et al., Accumulation of large very low density lipoprotein in plasma during intravenous infusion of a chylomicron-like triglyceride emulsion reflects competition for a common lipolytic pathway. J Lipid Res, 1996. 37(1): p. 76-86.

155. Kiens, B., et al., Effects of insulin and exercise on muscle lipoprotein lipase activity in man and its relation to insulin action. J Clin Invest, 1989. 84(4): p. 1124-9.

156. Kim, S.J., C. Nian, and C.H. McIntosh, Activation of lipoprotein lipase by glucosedependent insulinotropic polypeptide in adipocytes. A role for a protein kinase B, 
LKB1, and AMP-activated protein kinase cascade. J Biol Chem, 2007. 282(12): p. 8557-67.

157. Malmstrom, R., et al., Effects of insulin and acipimox on VLDL1 and VLDL2 apolipoprotein B production in normal subjects. Diabetes, 1998. 47(5): p. 779-87.

158. Walsh, B.W., et al., Effects of postmenopausal estrogen replacement on the concentrations and metabolism of plasma lipoproteins. N Engl J Med, 1991. 325(17): p. 1196-204.

159. Nordestgaard, B.G., et al., Nonfasting triglycerides and risk of myocardial infarction, ischemic heart disease, and death in men and women. JAMA, 2007. 298(3): p. 299-308.

160. Freiberg, J.J., et al., Nonfasting triglycerides and risk of ischemic stroke in the general population. JAMA, 2008. 300(18): p. 2142-52.

161. Bansal, S., et al., Fasting compared with nonfasting triglycerides and risk of cardiovascular events in women. JAMA, 2007. 298(3): p. 309-16.

162. Teno, S., et al., Association of postprandial hypertriglyceridemia and carotid intima-media thickness in patients with type 2 diabetes. Diabetes Care, 2000. 23(9): p. 1401-6.

163. Nakajima, K., et al., Postprandial lipoprotein metabolism: VLDL vs chylomicrons. Clin Chim Acta, 2011. 412(15-16): p. 1306-18.

164. Liu, L., et al., Remnant-like particles accelerate endothelial progenitor cells senescence and induce cellular dysfunction via an oxidative mechanism. Atherosclerosis, 2009. 202(2): p. 405-14.

165. Shin, H.K., et al., Remnant lipoprotein particles induce apoptosis in endothelial cells by $N A D(P) H$ oxidase-mediated production of superoxide and cytokines via lectin-like oxidized low-density lipoprotein receptor-1 activation: prevention by cilostazol. Circulation, 2004. 109(8): p. 1022-8.

166. Ferreira, A.C., et al., Postprandial hypertriglyceridemia increases circulating levels of endothelial cell microparticles. Circulation, 2004. 110(23): p. 3599-603.

167. Vogel, R.A., M.C. Corretti, and G.D. Plotnick, Effect of a single high-fat meal on endothelial function in healthy subjects. Am J Cardiol, 1997. 79(3): p. 350-4.

168. Patel, S., et al., Acute hypertriglyceridaemia in humans increases the triglyceride content and decreases the anti-inflammatory capacity of high density lipoproteins. Atherosclerosis, 2009. 204(2): p. 424-8.

169. Praet, S.F., et al., Influence of acute exercise on hyperglycemia in insulin-treated type 2 diabetes. Med Sci Sports Exerc, 2006. 38(12): p. 2037-44.

170. Manders, R.J., J.W. Van Dijk, and L.J. van Loon, Low-intensity exercise reduces the prevalence of hyperglycemia in type 2 diabetes. Med Sci Sports Exerc, 2010. 42(2): p. 219-25.

171. van Dijk, J.W., et al., Exercise and 24-h Glycemic Control: Equal Effects for All Type 2 Diabetic Patients? Med Sci Sports Exerc, 2012.

172. Oberlin, D.J., et al., One bout of exercise alters free-living postprandial glycemia in type 2 diabetes. Med Sci Sports Exerc, 2014. 46(2): p. 232-8.

173. van Dijk, J.W., et al., Exercise therapy in type 2 diabetes: is daily exercise required to optimize glycemic control? Diabetes Care, 2012. 35(5): p. 948-54. 
174. van Dijk, J.W., et al., Effect of moderate-intensity exercise versus activities of daily living on 24-hour blood glucose homeostasis in male patients with type 2 diabetes. Diabetes Care, 2013. 36(11): p. 3448-53.

175. Gillen, J.B., et al., Acute high-intensity interval exercise reduces the postprandial glucose response and prevalence of hyperglycaemia in patients with type 2 diabetes. Diabetes Obes Metab, 2012. 14(6): p. 575-7.

176. Kjaer, M., et al., Regulation of hepatic glucose production during exercise in humans: role of sympathoadrenergic activity. Am J Physiol, 1993. 265(2 Pt 1): p. E275-83.

177. Minuk, H.L., et al., Glucoregulatory and metabolic response to exercise in obese noninsulin-dependent diabetes. Am J Physiol, 1981. 240(5): p. E458-64.

178. Gill, J.M., et al., Effects of prior moderate exercise on exogenous and endogenous lipid metabolism and plasma factor VII activity. Clin Sci (Lond), 2001. 100(5): p. 517-27.

179. Heden, T.D., et al., Prior Exercise and Postprandial Incretin Responses in Lean and Obese Individual. Med Sci Sports Exerc, 2013.

180. Enevoldsen, L.H., et al., The combined effects of exercise and food intake on adipose tissue and splanchnic metabolism. J Physiol, 2004. 561(Pt 3): p. 871-82.

181. Lipp, R.W., et al., Effects of postprandial walking on delayed gastric emptying and intragastric meal distribution in longstanding diabetics. Am J Gastroenterol, 2000. 95(2): p. 419-24.

182. Heden, T.D., et al., Prior exercise and postprandial incretin responses in lean and obese individuals. Med Sci Sports Exerc, 2013. 45(10): p. 1897-905.

183. Dekker, M.J., et al., Exercise prior to fat ingestion lowers fasting and postprandial $V L D L$ and decreases adipose tissue IL-6 and GIP receptor mRNA in hypertriacylglycerolemic men. J Nutr Biochem, 2010. 21(10): p. 983-90.

184. Blom, P.C., et al., Modification by exercise of the plasma gastric inhibitory polypeptide response to glucose ingestion in young men. Acta Physiol Scand, 1985. 123(3): p. 367-8.

185. O'Connor, A.M., et al., The gastroenteroinsular response to glucose ingestion during postexercise recovery. Am J Physiol Endocrinol Metab, 2006. 290(6): p. E1155-61.

186. Ueda, S.Y., et al., Comparable effects of moderate intensity exercise on changes in anorectic gut hormone levels and energy intake to high intensity exercise. J Endocrinol, 2009. 203(3): p. 357-64.

187. Ueda, S.Y., et al., Changes in gut hormone levels and negative energy balance during aerobic exercise in obese young males. J Endocrinol, 2009. 201(1): p. 151-9.

188. Lund, M.T., et al., Meal induced gut hormone secretion is altered in aerobically trained compared to sedentary young healthy males. Eur J Appl Physiol, 2013. 113(11): p. 2737-47.

189. Weiss, E.P., et al., Postprandial Plasma Incretin Hormones in ExerciseTrainedversus Untrained Subjects. Med Sci Sports Exerc, 2014.

190. Solomon, T.P., et al., Improved pancreatic beta-cell function in type 2 diabetic patients after lifestyle-induced weight loss is related to glucose-dependent insulinotropic polypeptide. Diabetes Care, 2010. 33(7): p. 1561-6. 
191. Bloem, C.J. and A.M. Chang, Short-term exercise improves beta-cell function and insulin resistance in older people with impaired glucose tolerance. J Clin Endocrinol Metab, 2008. 93(2): p. 387-92.

192. Slentz, C.A., et al., Effects of exercise training intensity on pancreatic beta-cell function. Diabetes Care, 2009. 32(10): p. 1807-11.

193. Malin, S.K., et al., Pancreatic beta-cell function increases in a linear dose-response manner following exercise training in adults with prediabetes. Am J Physiol Endocrinol Metab, 2013. 305(10): p. E1248-54.

194. Solomon, T.P., et al., A low-glycemic index diet combined with exercise reduces insulin resistance, postprandial hyperinsulinemia, and glucose-dependent insulinotropic polypeptide responses in obese, prediabetic humans. Am J Clin Nutr, 2010. 92(6): p. 1359-68. 
APPENDIX A

\title{
CONSENT Form to PARTiCiPATE IN A ReSEARCh STUdY
}

\author{
InVESTigatoR's NAMe: Dr. JiLl A. KANALEY \\ PROJECT \# 1208507
}

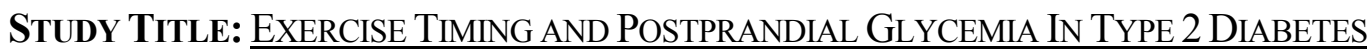

\section{INTRODUCTION}

This consent may contain words that you do not understand. Please ask the investigator or the study staff to explain any words or information that you do not clearly understand.

This is a research study. Research studies include only people who choose to participate. As a study participant you have the right to know about the procedures that will be used in this research study so that you can make the decision whether or not to participate. The information presented here is simply an effort to make you better informed so that you may give or withhold your consent to participate in this research study.

Please take your time to make your decision and discuss it with your family and friends.

You are being asked to take part in this study because you have type 2 diabetes (T2D).

In order to participate in this study, it will be necessary to give your written consent.

\section{Why Is This StUdy BeING DONE?}

The purpose of this study is to determine how the timing of resistance exercise around a dinner meal (before dinner vs. after dinner) alters blood glucose levels, hormonal responses, and gastric emptying in patients with T2D.

Patients with T2D have abnormally high blood glucose levels after a meal, and prior research has shown that exercise can help lower blood glucose levels after a meal. The current exercise guidelines for patients with T2D does not include the best time, relative to a meal (either before or after a meal), an individual with T2D should exercise to get the most benefit on their blood glucose levels. Thus, the scientific reason for doing this study is to determine if there is an optimal time for an individual with T2D to exercise to lower the blood glucose response to a meal.

\section{How Many People Will Take Part In The Study?}

About 15 people will take part in this study at the University of Missouri. 


\section{WHAT IS INVOLVED IN THE STUDY?}

Visit 1 - Screening ( $\sim 30 \mathrm{~min})$ : You will need to come in initially to have some tests done to determine your eligibility for the study. At this visit you will:

- Review and sign the consent form.

- Height, weight and blood pressure measurement

- Fasting glucose levels and HbAlc: We will ask you to come in after an overnight fast to the Exercise Physiology Lab in McKee Gym to have your fasting blood glucose checked in the morning, as well as get a measure of your HbAlc. This will be done by getting a blood sample by finger stick.

- Complete a health inventory questionnaire.

You will be told at the end of the screening if you qualify or not. In order to participate in this study, you will need to receive permission from your physician to exercise.

Visit 2 - Resting energy expenditure (REE), body composition (BODPOD), and familiarization session one.

You will need to overnight fast for $\sim 10 \mathrm{~h}$ prior to Visit 2 . This testing will be in the morning period.

REE: During the REE, you will lay supine in a recliner for 30 min while all expired air is collected in a canopy that will be placed over your head and analyzed for $\mathrm{O}_{2}$ and $\mathrm{CO}_{2}$ content. You will be able to breathe room air normally during the procedure. Expired air will be collected and analyzed in the machine.

Body Composition: In this test we will measure your percent body fat using the BOD POD. In this test you will wear a bathing suit (or tight fitting shorts/T-shirt) and sit in capsule quietly while the machine makes the measurement. The BOD POD is like a large bubble about 5' high and 4'wide with a window in it. We ask you to sit quietly inside the Pod, while we make the measures of how much space you take up in the BOD POD. You will then be asked to puff gently through a tube a few times so we can determine how much air is in your lungs. During this test you will not feel anything and you should not feel any anxiety about sitting in the BOD POD as there is a window and you can the investigator during the testing. This part of the testing takes about 10 minutes.

Familiarization session one: This session will be used to teach you how to properly perform each exercise. The weight for the resistance exercises will be light $(\sim 10-40 \%$ of bodyweight) and you will perform four sets of 10 repetitions with a 1 min rest period between each set of the following exercises (performed in this order): 1) Leg Press, 2) Calf Raises, 3) Chest Flyes, 4) Back Flyes, 5) Back Extensions, 6) Shoulder Raises, 7) Hamstring Curls, and 8) Abdominal Crunches.

Visit 3 - Strength Testing

There will be at least a $3 \mathrm{~d}$ break after the first exercise session, and after this you will report back to the lab. Your 10 repetition max (10-RM) will be determined for each exercise. The 10-RM is the maximum amount of weight that you can lift 10 times. During this session, each exercise will begin with a warm-up set $(\sim 10-40 \%$ of bodyweight), and then for each subsequent set weight will be progressively added until 
the 10-RM for the exercise is determined. At least 2 min of rest will be allowed between each 10-RM attempt.

Visit 4 - Familiarization session two.

Following a $3 \mathrm{~d}$ break, you will report back to the lab. On this visit, you will perform four sets ( 1 min rest between sets) of 10 repetitions of the exercises described previously. The first set for each exercise will be a warm-up set and the weight used will be $50 \%$ of your 10-RM. After the warm-up set, the weight for the next three sets will be $80 \%$ of your 10 RM.

Visits 5-13: You will complete three different trials. Each trial will be 3 days in length and separated by at least 1 week. The three trials will include 1) a no exercise trial, 2) exercise before dinner, and 3) exercise after dinner.

Day 1: During the evening of day one of each trial $(1800 \mathrm{~h})$ you will report to the lab and a continuous glucose monitor system (CGMS) will be inserted and you will be trained on how to take and record a finger stick blood glucose measure. You will be given prepackaged study meals and will be instructed on when to eat the meals.

- The CGMS allows us to gain information about how your blood sugar levels change over the course of a day in a free living setting. The CGMS is worn on your abdomen and it is a tiny glucose sensor which is inserted just under the skin. We will clean the area, and then a small tube (catheter) $\sim 1 / 2$ the size of a sewing needle will be placed under your skin. The insertion is quick, and is usually not painful. The tube is attached to a wire connected to a small device approximately the size if a silver dollar, and this device measures and records your blood glucose values throughout the day. Tape holds everything in place. In addition, you will be asked to make at least four finger stick blood glucose readings with a standard glucose meter at different times each day. The monitor will be removed after testing. Any exercise engaged in, and meals or snacks consumed are also entered into a paper-based diary. After the first time you wear the CGMS, we will ask you to repeat the timing, content and quantity of food consumption to match your first visit.

Day 2: You will eat the prepackaged meals at the scheduled times on your own in the morning and afternoon. Prior to each meal, you will take and record a finger stick blood glucose measure.

This day will last $\sim 6 \mathrm{hr}$.

- You will arrive at 4:15 pm, and return your food containers.

- Height, weight and resting blood pressure will be measured.

- A small catheter will be placed in the forearm vein so that we can draw blood samples. This catheter will be attached to an IV of saline water which will keep this line open after each blood sample. This allows us to take multiple blood samples but not have to stick you repeatedly. This remains in your arm until 10 pm.

- Appetite ratings will be taken throughout the study evening.

- You will consume a dinner at $6 \mathrm{pm}$, and will be given $20 \mathrm{~min}$ to eat the meal. Acetaminophen will be ground up and put into the spaghetti sauce of the dinner 
meal so that gastric emptying can be measured. You will not be able to taste the acetaminophen.

- No exercise trial: you will remain physically inactive during the time you are in the lab.

- Exercise trial 1: $\sim 45$ min of resistance exercise will be performed between 4:45 $5: 30 \mathrm{pm}$

- Exercise trial 2: $\sim 45 \mathrm{~min}$ of resistance exercise will be performed $30 \mathrm{~min}$ after completing dinner.

- The order of these 3 trials will be randomized.

- Energy expenditure will be measured using the metabolic cart between 1645-1750 $\mathrm{h}, 1850-1935 \mathrm{~h}$, and 2100-2130 h.

- Blood samples will be taken throughout the evening. You can drink as much water as you like during this study day. You will sit quietly during the day and can read, watch DVDs, etc. except when testing for energy expenditure. During this testing you must remain still and it must be quiet or you will be exercising.

Day 3: You will report back to the lab at $\sim 0800 \mathrm{~h}$ and a fasting blood sample will be taken and you will be given breakfast.

\section{How Long Will I Be in the Study?}

The study will take at least 5 weeks to complete.

You can stop participating at any time. Your decision to withdraw from the study will not affect in any way your medical care and/or benefits.

\section{WHAT ARE THE RISKS OF THE STUDY?}

Risks of body composition: The BODPOD is safe, although you may experience claustrophobia. A window is built into the machine that allows you to look outward to help avoid the feeling of claustrophobia and there is a release button on the inside of the machine should you feel like you must get out.

Risks of blood sampling: May cause fainting and some discomfort and/or bruising at the site on the arm where the blood was taken. Rarely an infection occurs at the blood drawing site. There is also a risk of a blood clot forming in the vein and a risk of developing anemia.

Risks of acetaminophen: You will be given a dose of acetaminophen mixed in with the dinner meal ( $2 \mathrm{~g}$ which will be grounded up and added into the spaghetti sauce for the dinner). This dose of acetaminophen will not exceed the daily recommended dose of 4-5 g. Risks of acetaminophen administration are rare but can include nausea, vomiting, and at high repeated doses liver or kidney damage. Thus, you should not consume over-thecounter or prescription medications containing acetaminophen the day before or the day after the study. Over-the-counter medications to avoid include: Tylenol, Exedrin PM, Alka Seltzer Plus Cold, TheraFlu cold medicine, while prescription medications for pain include Vicodin and Percocet.

Risks of resistance training: Resistance training may cause skeletal muscle soreness. This is not permanent and will resolve within a few days.

CGMS Glucose monitoring: There is a small risk of infection, bruising or nausea associated when using the CGMS. To minimize risk, the placement of the CGMS is performed with sterile techniques by qualified personnel in the Exercise Physiology Lab. 


\section{Are There Benefits to Taking Part in the StUdy?}

The data generated from this project will be useful for patients with type 2 diabetes and will highlight the best time to perform exercise to lower their blood glucose responses after dinner. Potentially this study may motivate some subjects to start exercising in the evening.

\section{What Other Options Are There?}

The alternative is to not participate in this study.

\section{WHAT ABOUT CONFIDENTIALITY?}

Information produced by this study will be stored in the investigator's file and identified by a code number only. The code key connecting your name to specific information about you will be kept in a separate, secure location. Information contained in your records may not be given to anyone unaffiliated with the study in a form that could identify you without your written consent, except as required by law. If the investigator conducting this study is not your primary, or regular doctor, she must obtain your permission before contacting your regular doctor for information about your past medical history or to inform them that you are in this trial.

It is possible that your medical and/or research record, including sensitive information and/or identifying information, may be inspected and/or copied by the study sponsor (and/or its agent), the Food and Drug Administration (FDA), federal or state government agencies, or hospital accrediting agencies, in the course of carrying out their duties. If your record is inspected or copied by the study sponsor (and/or its agents), or by any of these agencies, the University of Missouri will use reasonable efforts to protect your privacy and the confidentiality of your medical information.

The results of this study may be published in a medical book or journal or used for teaching purposes. However, your name or other identifying information will not be used in any publication or teaching materials without your specific permission.

\section{What ARE THE Costs?}

The only cost to you may be transportation to/from our research laboratory.

\section{Will I be PAid FOR PARTicipating In The Study?}

You will be compensated $\$ 180.00$ for completion of this study.

If you do not complete the study days and drop out, you will be paid $\$ 7 / \mathrm{hr}$ for the time you put in.

\section{WHAT IF I AM INJURED?}

It is not the policy of the University of Missouri to compensate human subjects in the event the research results in injury. The University of Missouri, in fulfilling its public responsibility, has provided medical, professional and general liability insurance coverage for any injury in the event such injury is caused by the negligence of the University of 
Missouri, its faculty and staff. The University of Missouri also will provide, within the limitations of the laws of the State of Missouri, facilities and medical attention to subjects who suffer injuries while participating in the research projects of the University of Missouri. In the event you have suffered injury as the result of participation in this research program, you are to contact the Risk Management Officer, telephone number (573) 882-1181, at the Health Sciences Center, who can review the matter and provide further information. This statement is not to be construed as an admission of liability.

\section{What ARe My Rights as A PARTiCipant?}

Participation in this study is voluntary. You do not have to participate in this study. Your present or future care will not be affected should you choose not to participate. If you decide to participate, you can change your mind and drop out of the study at any time without affecting your present or future care in the University of Missouri hospital. Leaving the study will not result in any penalty or loss of benefits to which you are entitled. In addition, the investigator of this study may decide to end your participation in this study at any time after she has explained the reasons for doing so and hashelped arrange for your continued care by your own doctor, if needed. You will be informed of any significant new findings discovered during the course of this study that might influence your health, welfare, or willingness to continue participation in this study.

\section{Whom do I Call if I Have Questions or Problems?}

If you have any questions regarding your rights as a participant in this research and/or concerns about the study, or if you feel under any pressure to enroll or to continue to participate in this study, you may contact the University of Missouri Health Sciences Institutional Review Board (which is a group of people who review the research studies to protect participants' rights) at (573) 882-3181. You may ask more questions about the study at any time. For questions about the study or a research-related injury, contact Jill Kanaley, $\mathrm{PhD}$ at 573-882-2519.

A copy of this consent form will be given to you to keep.

\section{SIGNATURE}

I confirm that the purpose of the research, the study procedures, the possible risks and discomforts as well as potential benefits that I may experience have been explained to me. Alternatives to my participation in the study also have been discussed. I have read this consent form and my questions have been answered. My signature below indicates my willingness to participate in this study.

Subject/Patient*

Date

\section{SIGNATURE OF STUDY REPRESENTATIVE}

I have explained the purpose of the research, the study procedures, identifying those that are investigational, the possible risks and discomforts as well as potential benefits and have answered questions regarding the study to the best of my ability. 
****Study Representative is a person authorized to obtain consent. Per the policies of the University of Missouri Health Care, for any 'significant risk/treatment' study, the Study Representative must be a physician who is either the Principal or Co-Investigator. If the study is deemed either 'significant risk/non-treatment' or 'minimal risk,' the Study Representative may be a non-physician study investigator. 


\begin{abstract}
APPENDIX B
Manuscript, will be submitted to journal "Diabetologia".

Post-dinner resistance exercise is more effective at improving postprandial metabolic risk factors than pre-dinner resistance exercise in obese patients with type-2 diabetes
\end{abstract}

Timothy D. Heden ${ }^{1}$, Nathan C. Winn ${ }^{1}$, Andrea Mari ${ }^{2}$, Frank W. Booth ${ }^{1,3}$, R. Scott Rector $^{1,4,5}$, John P. Thyfault ${ }^{1,4,5}$, Jill A. Kanaley ${ }^{1}$

${ }^{1}$ Department of Nutrition and Exercise Physiology, University of Missouri, Columbia, MO

${ }^{2}$ Institute of Biomedical Engineering, National Research Council, Padova, Italy

${ }^{3}$ Department of Biomedical Sciences, University of Missouri, Columbia, MO

${ }^{4}$ Internal Medicine - Division of Gastroenterology and Hepatology, University of

Missouri, Columbia, MO

${ }^{5}$ Research Service, Harry S. Truman Memorial VA Hospital, University of Missouri, Columbia, MO.

Corresponding Author:

Jill A. Kanaley, PhD

Professor and Associate Chair

Department of Nutrition and Exercise Physiology

204D Gwynn Hall, University of Missouri, Columbia, MO 65211

Phone \#: 573.882.2519

Fax \#: 573.884.4885

Email: kanaleyj@missouri.edu

Word Count-Abstract: 250

Word Count-Main Text: 3,980 (including Figure and Table legends) 


\begin{abstract}
Abnormally elevated postprandial glucose and triacylglycerol (TAG) concentrations are risk factors for cardiovascular disease in patients with type- 2 diabetes. The most effective time to exercise to lower postprandial glucose and TAG concentrations is unknown. Thus, the aim of this study was to determine what time is more effective, either pre- or post-dinner resistance exercise (RE), at improving postprandial risk factors in patients with type-2 diabetes. Methods: Thirteen obese-patients with type-2 diabetes completed three trials in a random order in which they consumed a dinner meal with 1) no RE (NoRE), 2) pre-dinner $\mathrm{RE}(\mathrm{RE} \rightarrow \mathrm{M})$, and 3) post-dinner $\mathrm{RE}$ beginning 45-min after dinner $(\mathrm{M} \rightarrow \mathrm{RE})$. Clinical outcome measures included postprandial glucose and TAG concentrations. In addition, postprandial acetaminophen (gastric emptying), endocrine responses, FFA, and beta-cell function (mathematical modeling) were measured to determine if alterations in these factors were related to changes in glucose and TAG. Results: The TAG incremental-AUC (iAUC) was $\sim 92 \%$ lower $(\mathrm{P}<0.05)$ during $\mathrm{M} \rightarrow \mathrm{RE}$ compared to NoRE and $\mathrm{RE} \rightarrow \mathrm{M}$, an effect due in part to lower VLDL-1 TAG concentrations. The glucose iAUC was reduced $(\mathrm{P}<0.05)$ by $\sim 18 \%$ and $30 \%$ during the $\mathrm{RE} \rightarrow \mathrm{M}$ and $\mathrm{M} \rightarrow \mathrm{RE}$ trials, respectfully, compared to NoRE, with no difference between $\mathrm{RE}$ trials. $\mathrm{RE} \rightarrow \mathrm{M}$ and $\mathrm{M} \rightarrow \mathrm{RE}$ reduced the insulin $i \mathrm{AUC}$ by $35 \%$ and $48 \%$, respectfully, compared to NoRE $(\mathrm{P}<0.05)$. The GLP-1 iAUC was $~ 50 \%$ lower $(\mathrm{P}<0.05)$ during $\mathrm{M} \rightarrow \mathrm{RE}$ compared to NoRE and $\mathrm{RE} \rightarrow \mathrm{M}$. No other variables were different between trials. Conclusions: Post-dinner RE improves postprandial metabolic risk factors better than pre-dinner RE in obese patients with type-2 diabetes.
\end{abstract}


Key words: Exercise timing, glycaemic control, weight training, obesity, glucose metabolism, lipid metabolism

\section{Introduction}

Abnormally elevated postprandial glucose [1-3] and triacylglycerol (TAG) [4-6] concentrations are strong risk factors for cardiovascular disease (CVD) in patients with type-2 diabetes. Therefore, interventions that reduce postprandial glucose and TAG concentrations should lower the risk of CVD [7-8]. Acute exercise typically lowers postprandial glucose [9-19] and TAG concentrations [20] in patients with type-2 diabetes, but there is considerable heterogeneity in the responses with some individuals not experiencing beneficial changes in these risk factors $[13,21]$. One potential explanation why some patients with type-2 diabetes do not have beneficial changes in postprandial glucose and TAG with acute exercise is because of the timing of the acute exercise session relative to a meal.

Limited evidence suggests that the timing of aerobic exercise around a meal may be important and might explain why some individuals are exercise "insensitive" or "nonresponders". The only study to directly compare pre-meal and post-meal exercise in patients with type-2 diabetes showed that post-dinner, but not pre-dinner walking, lowered postprandial glucose concentrations [22]. Although no study has directly examined the effect of exercise timing on postprandial TAG in patients with type-2 diabetes, there is evidence that exercise performed the day prior to a high fat meal has no effect on postprandial TAG responses [21, 23], while post-breakfast aerobic exercise reduced the postprandial TAG response [20]. Taken together, it appears that aerobic exercise may have its most powerful effect to lower postprandial glucose and TAG 
responses when performed after a meal, possibly because of reduced gastric emptying and/or greater skeletal muscle glucose and TAG uptake and utilization at this time.

The aforementioned studies examined aerobic exercise, but no study, to our knowledge, has examined how resistance exercise (RE) timing relative to dinner alters postprandial glucose and TAG concentrations. From a practical perspective, many obese patients with type-2 diabetes may not enjoy or be able to perform aerobic exercise soon after a meal when the exercise session may have its most powerful effect to lower postprandial glucose and TAG concentrations. However, RE may be a practical alternative because it can be better tolerated in obese patients with type- 2 diabetes because traditional RE consists of short work periods with long rests between sets. However, compared to aerobic exercise, RE is a different physiological stimulus (i.e. RE is anaerobic, does not involve continuous skeletal muscle contractions, and has lower energy expenditure), thus it is not clear if RE would alter postprandial glucose and TAG concentrations as robustly as aerobic exercise. Some studies report acute RE prior to meal(s) improves glycaemic control [18-19], while other studies report it does not [24-25], and no study has assessed how acute RE alters postprandial TAG concentrations. In addition, these studies assessed the effects of RE on postprandial glucose concentrations after a breakfast or lunch meal, and not a dinner meal later in the day, a time when glucose and TAG concentrations have been reported to be highest in patients with type-2 diabetes [26-27]. Therefore, the primary purpose of this study was to test the hypothesis that post-dinner RE, compared to pre-dinner RE, is more effective at improving two clinically important postprandial risk factors (glucose and TAG) for CVD at a time of day when they are typically highest in obese patients with type-2 diabetes. In addition, postprandial acetaminophen (gastric 
emptying), endocrine responses, free fatty acids (FFA), and beta-cell function (mathematical modeling) were measured to determine if alterations in these factors were related to changes in postprandial glucose and TAG concentrations. Subjective wellbeing was assessed to determine how feasible the different RE times were.

\section{Research Design and Methods}

\section{Participants}

The University of Missouri Health Science Institutional Review Board approved this study protocol and all participants provided written informed consent. Participants in this study were obese (body mass index $>30 \mathrm{~kg} / \mathrm{m}^{2}$ ), physician diagnosed with type 2 diabetes, receiving standard medical care, non-smokers, not using insulin, had no history of surgery for weight loss, refrained from performing exercise or going on any special diets while participating, and were weight stable. The participants took their medications at the usual dose, frequency, and time while participating in this study.

\section{Experimental Design}

Baseline testing included assessments of height, weight, body composition (assessed via $\left.\mathrm{BODPOD}{ }^{\circledR}\right)$, resting energy expenditure, physical activity energy expenditure, and familiarization and strength testing (described in detail later). After baseline testing, all participants completed three, $3 \mathrm{~d}$ trials in a random order. On day one a continuous glucose monitor (CGM) was inserted into the abdomen of the participant and the participants were instructed on how to take and record a finger stick blood glucose measure with a glucometer (Accu-Chek Compact Plus, Roche Diagnostics), which was used to calibrate the CGM. Following training, the participants were given their prepackaged study meals and were instructed on when to eat the meals the following 
day. On day two, the participants ate the standardized breakfast and lunch on their own and immediately prior to each meal recorded a finger stick blood glucose measure. In the evening the participants reported to the lab for testing sometime between $3-5: 30 \mathrm{p} . \mathrm{m}$. and upon arrival a venous catheter was inserted into a forearm vein. The participants consumed a dinner meal in the lab with 1) no RE (NoRE), 2) pre-dinner RE (RE $\rightarrow \mathrm{M})$ ending 20-30 min before dinner, or 3) post-dinner RE beginning 45 min after dinner $(\mathrm{M} \rightarrow \mathrm{RE}$ ). On day three of all trials (between 6:30-8:30a.m.) and $\sim 12-15 \mathrm{~h}$ after the RE session the participants reported to the lab overnight fasted and a fasting blood sample was taken, followed by consumption of a standardized breakfast meal. After the participants consumed breakfast they were allowed to leave the lab but were required not to consume any food for $4 \mathrm{~h}$ so that the $4 \mathrm{~h}$ postprandial glucose response to breakfast could be measured.

\section{Resistance Exercise}

For baseline testing, each participant performed familiarization strength testing so that they were not naive to RE before the study. Participants completed the first familiarization to teach them how to properly perform each exercise. During this visit, the weight for RE was light ( $10-40 \%$ of bodyweight) and the participants performed $1-2$ sets of 10-repetitions of the following exercises (in this order): Leg press, seated calf raises, seated chest flyes, seated back flyes, back extensions, shoulder raises, leg curls, and abdominal crunches. Within a week of this first visit, participants returned for strength testing and their 10 repetition maximum (10-RM) for each exercise described previously (except abdominal crunches) was determined. At least $3 \mathrm{~d}$ after 10-RM testing, a second familiarization was performed during which the participants performed 
three sets (1-2 min rest between sets) of 10-repetitions. During this session, the first set for each exercise was a warm-up set and the weight used was $50 \%$ of the participants 10 RM. After the warm-up set, the weight for the next two sets was the participants previously determined 10-RM. Following these visits, the participants completed the three study days and the RE session during the study days was identical to the protocol for the second familiarization session.

Diet

During baseline testing, resting energy expenditure was measured using indirect calorimetry (ParvoMedics TrueOne ${ }^{\circledR} 2400$ ) and physical activity energy expenditure was measured with a BodyMedia ${ }^{\circledR}$ armband (BodyMedia, Inc). The sum of these two measurements was used as an estimate of total daily energy expenditure (TDEE) and the participants were provided with this much energy during the day of testing. For all meals, the percent of energy from each macronutrient was $\sim 50 \%$ carbohydrate, $35 \%$ fat, and $15 \%$ protein. The breakfast meal was $2,448 \mathrm{kj}$, the lunch meal was $2,439 \mathrm{kj}$, and the dinner meal was a spaghetti dinner with $1.5 \mathrm{~g}$ of acetaminophen (to assess gastric emptying). The energy content of dinner was calculated by subtracting 4,962 $\mathrm{kj}$ (from breakfast and lunch meals) from the estimated TDEE for each participant.

Indirect Calorimetry, Heart Rate, Ratings of Perceived Exertion, and Well-being

Indirect calorimetry was used to measure energy expenditure and substrate oxidation during the time frame when RE was performed and for $\sim 15-30$ min starting $\sim 3.5 \mathrm{~h}$ after dinner. Ratings of perceived exertion were measured at the end of every RE set using the BORG 6-20 scale. Subjective well-being was assessed using a 100-mm visual analog scale after every blood draw to determine how RE made the participants 
feel. The question was worded "How strong is your overall feeling of well-being (pleasure)" and the participants marked a single horizontal line through the vertical line on the scale in-between the anchors "not at all" and "extremely".

\section{Blood Collection}

Blood samples were taken every 5-10 min during the first $3.7 \mathrm{~h}$ of testing and every 30 min during the last $2 \mathrm{~h}$. Blood samples were transferred immediately into chilled EDTA tubes either pretreated with Aprotinin (ThermoFisher Scientific, Inc.) only (for TAG samples), chilled EDTA tubes pretreated with dipeptidyl peptidase-4 inhibitor (Millipore Corp.), Pefabloc ${ }^{\circledR}$ SC (DSM Nutritional Products AG), and Aprotinin (for hormone, glucose, and FFA analysis), or EDTA tubes not treated with any protease inhibitors (for acetaminophen assay). Blood was separated by centrifugation using an Eppendorf 5702R centrifuge at 3,000 RPM for $10 \mathrm{~min}$ at $4^{\circ} \mathrm{C}$, and then frozen at $-80^{\circ} \mathrm{C}$ until analysis.

\section{Separation of Lipoprotein Species}

Separation of plasma chylomicron ( $\left.\mathrm{S}_{\mathrm{f}}>400\right)$, VLDL-1 ( $\left.\mathrm{S}_{\mathrm{f}} 60-400\right)$, and VLDL-2 ( $\mathrm{S}_{\mathrm{f}}$ 20-60) was performed using density gradient ultracentrifugation, as described by Karpe et al. [28], but with minor modification. $\mathrm{KBr}(0.1 \mathrm{~g})$ was mixed into $1 \mathrm{~mL}$ of fresh plasma to bring the density up to $\sim 1.1 \mathrm{~g} / \mathrm{L}$ in a polyallomer ultracentrifuge tube (14 x 89 mm. tube, Beckman Coulter, Inc.). Next, a density gradient consisting of $3 \mathrm{ml}$ of $\mathrm{KBr}$ salt solution $(1.1 \mathrm{~g} / \mathrm{l}), 4 \mathrm{ml}$ of $\mathrm{NaCl}$ solution $(1.065 \mathrm{~g} / \mathrm{l}), 3 \mathrm{ml}$ of $\mathrm{KBr}$ solution (density 1.020 $\mathrm{g} / \mathrm{l}$ ), and $1 \mathrm{ml}$ of $\mathrm{NaCl}$ solution (density $1.006 \mathrm{~g} / \mathrm{l}$ ) was layered above the plasma. Ultracentrifugation was performed using a TH-641 (ThermoFisher Scientific, Inc.) swinging bucket rotor at 40,000 RPM and $10^{\circ} \mathrm{C}$. For the separation of each lipoprotein 
species, consecutive ultracentrifugations of $32 \mathrm{~min}$ (chylomicrons), $3 \mathrm{~h}$ and $28 \mathrm{~min}$ (VLDL-1), and $16 \mathrm{~h}$ (VLDL-2) were performed. After each run, the top $1 \mathrm{~mL}$ layer was carefully removed and frozen at $-80^{\circ} \mathrm{C}$ until analysis. Before the next run, the tube was refilled with $1 \mathrm{~mL}$ of $\mathrm{NaCl}$ solution of density $1.006 \mathrm{~g} / \mathrm{l}$. Only five time points were assessed for TAG due to limited space in the ultracentrifuge.

\section{Biochemical Analyses}

Whole blood glucose was assessed using a YSI 2700 Select (YSI Incorporated). Plasma TAG (Infinity ${ }^{\mathrm{TM}}$, ThermoFisher Scientific Inc.), free fatty acids (Wako Chemicals Inc.), and acetaminophen (Cambridge Life Science LTD) concentrations were determined using a colorimetric assay. Plasma hormone concentrations were determined using a MILLIPLEX magnetic bead-based immunoassay (Millipore Corp.). Hematocrit was measured after every blood draw and samples were corrected for plasma volume shifts. Plasma volume variations $(\Delta \mathrm{VP})$ were calculated from hematocrit variations $(\mathrm{Ht}, \mathrm{Ht} 1=$ baseline $\mathrm{Ht}, \mathrm{Ht} 2=$ after baseline $\mathrm{Ht})$ using the following formula: $\% \Delta \mathrm{VP}=100 *[(\mathrm{Ht} 1-$ $\mathrm{Ht} 2) /(\mathrm{Ht} 2 *(100-\mathrm{Ht} 1))]$. Corrected plasma values were calculated using the following formula: Corrected Value $=($ Initial Value*100 $) /(100-\Delta V P)$

Beta-cell function

Model based beta-cell function parameters (beta-cell glucose sensitivity, potentiation factor ratio, and rate sensitivity) were calculated using a mathematical model developed by Mari et al. [29, 30]. Briefly, beta-cell glucose sensitivity is the slope of the dose response relation between insulin secretion, glucose concentration, and the potentiation factor. The potentiation factor ratio is the ratio between potentiation at the end of the dinner meal (220-240 $\mathrm{min})$ and the initial value $(0-20 \mathrm{~min})$. Rate sensitivity 
represents the enhancement of insulin secretion proportional to the rate of change of plasma glucose. Insulin secretion rates (ISR) used in the model were calculated by deconvoluting c-peptide concentrations [31].

\section{Calculations}

Postprandial responses were quantified using incremental area under the curve (iAUC) [32]. Gastric emptying was quantified using the acetaminophen iAUC. Insulin clearance was calculated as the molar ratio of insulin to c-peptide at each time point as a percent $((1$-[insulin/c-peptide pmol/L])*100). The change in glucose and TAG iAUC was calculated by subtracting the iAUC during the NoRE trial from the iAUC during either the $\mathrm{M} \rightarrow \mathrm{RE}$ or $\mathrm{RE} \rightarrow \mathrm{M}$ trial. Glycemic variability was calculated using standard deviation and continuous overall net glycemic action (CONGA-1, -2, and -4) [33].

Statistical Analysis

Statistical analyses were performed using the Statistical Package for the Social Sciences statistical software, version 18.0 (IBM Inc.). A paired samples t-test was used to compare metabolic, heart rate, and perceived exertion data between trials. A repeated measures ANOVA with follow-up Bonferroni adjusted post hoc t-tests was used to determine specific differences between iAUC values. Alpha was set at $\mathrm{P} \leq 0.05$. All values are reported as means \pm S.E.M. unless otherwise denoted.

\section{Results}

\section{Participants}

Thirteen obese men and women with type 2 diabetes completed this study (Table 1). The participants were weight stable while enrolled in this study. Metabolic and Perceived Exertion Data During Exercise and Post Dinner 
The average heart rate was 4 beats per minute higher $(\mathrm{P}<0.05)$ during $\mathrm{M} \rightarrow \mathrm{RE}$ compared to $\mathrm{RE} \rightarrow \mathrm{M}$, while all other variables were similar (Table 2, $\mathrm{P}>0.05$ ). Postprandial energy expenditure and substrate oxidation $\sim 3.5-4 \mathrm{~h}$ after dinner was not significantly different between trials (Table 3, $\mathrm{P}>0.05$ ).

Glucose, Triacylglycerol, and FFA Responses

The pre-dinner glucose iAUC was significantly lower $(\mathrm{P}<0.05)$ during $\mathrm{RE} \rightarrow \mathrm{M}$ compared to NoRE and $\mathrm{M} \rightarrow \mathrm{RE}$ (Figure 1B). The postprandial glucose iAUC was reduced by $30 \%$ and $18 \%$ during $M \rightarrow R E$ and $R E \rightarrow M$, respectively, compared to NoRE (both $\mathrm{P}<0.05$ ), with no difference between exercise trials (Figure 1C). During $\mathrm{M} \rightarrow \mathrm{RE}$, the postprandial $4 \mathrm{~h}$ total TAG iAUC was reduced by $\sim 92 \%(\mathrm{P}<0.05)$ compared to NoRE and $\mathrm{RE} \rightarrow \mathrm{M}$ (Figure 2B), an effect due to reduced VLDL-1 TAG concentrations (Figure 3D). Neither chylomicron nor VLDL-2 TAG concentrations were different between trials $(\mathrm{P}>0.05)$. The rise in pre-meal FFA concentrations was significantly attenuated $(\mathrm{P}<0.05)$ during $\mathrm{RE} \rightarrow \mathrm{M}$ compared to $\mathrm{NoRE}$ and $\mathrm{M} \rightarrow \mathrm{RE}$ (Figure 4B). Similarly, the drop in postprandial FFA concentrations was significantly less $(\mathrm{P}<0.05)$ during $\mathrm{RE} \rightarrow \mathrm{M}$ compared to NoRE and $\mathrm{M} \rightarrow \mathrm{RE}$ (Figure 4C).

Pancreatic and Gut Hormone Responses

Pre-dinner insulin concentrations were not different between trials, but the postprandial insulin iAUC was $39 \%$ and $31 \%$ lower $(\mathrm{P}<0.05)$ during $\mathrm{M} \rightarrow \mathrm{RE}$ and $\mathrm{RE} \rightarrow \mathrm{M}$, respectively, compared to NoRE (Figure 5C). The pre-dinner c-peptide iAUC was $\sim 2.7$ fold lower $(\mathrm{P}<0.05)$ during $\mathrm{RE} \rightarrow \mathrm{M}$ compared to $\mathrm{M} \rightarrow \mathrm{RE}$ and NoRE (Figure 5E). The postprandial c-peptide iAUC was $45 \%$ lower $(\mathrm{P}<0.05)$ during $\mathrm{M} \rightarrow \mathrm{RE}$ compared to NoRE (Figure 5F). Pre-meal glucagon responses were not different between 
trials but the postprandial glucagon iAUC dropped significantly more $(\mathrm{P}<0.05)$ during $\mathrm{RE} \rightarrow \mathrm{M}$ compared to the other trials because it was higher at the start of the meal (Figure 5I). Neither pre-meal nor postprandial GIP concentrations were significantly different between trials (Figure 6A). During M $\rightarrow$ RE, the postprandial GLP-1 iAUC was $50 \%$ and 49\% lower $(\mathrm{P}<0.05)$ compared to $\mathrm{RE} \rightarrow \mathrm{M}$ and NoRE, respectfully (Figure 6F).

Insulin Kinetics, Beta-cell Function, and Gastric Emptying

The postprandial ISR iAUC was $40 \%$ lower $(\mathrm{P}<0.05)$ during the $\mathrm{M} \rightarrow \mathrm{RE}$ trial compared to the NoRE trial (Figure 7B). The postprandial insulin clearance iAUC was nearly 3 fold and 2 fold greater $(P<0.05)$ during $R E \rightarrow M$ and $M \rightarrow R E$, respectfully, compared to NoRE and nearly 2 fold greater $(\mathrm{P}<0.05)$ during $\mathrm{RE} \rightarrow \mathrm{M}$ compared to $M \rightarrow R E$ (Figure 7D). There were no significant differences ( $P>0.05)$ in gastric emptying, beta-cell glucose sensitivity, rate sensitivity, or the potentiation factor ratio between trials (Table 4).

Glycemic Variability, Nocturnal and Morning Glycaemic Control, and Insulin Sensitivity

Glycaemic variability over the $24 \mathrm{~h}$ period between the beginning of lunch on day 2 and day 3 of each trial was not significantly different $(\mathrm{P}>0.05)$ between trials (Table 5). Furthermore, nocturnal or morning glycaemic control (Table 6), fasting glucose, insulin, c-peptide, insulin clearance, HOMA-IR, QUICKI, and TAG (Table 7) were not different between trials the next morning $(\mathrm{P}>0.05)$.

\section{Subjective Well-being}

Baseline well-being was significantly different between trials $(\mathrm{P}<0.05)$, thus to better illustrate the change in well-being, change scores were used. The pre-meal subjective well-being $i \mathrm{AUC}$ was significantly greater during $\mathrm{RE} \rightarrow \mathrm{M}$ compared to NoRE 
$(\mathrm{P}<0.05)$ and tended to be greater $(\mathrm{P}=0.064)$ compared to $\mathrm{M} \rightarrow \mathrm{RE}$ (Figure 8B). The postprandial well-being iAUC was significantly greater $(\mathrm{P}<0.05)$ with $\mathrm{RE}$ compared to NoRE, independent of RE timing (Figure 8D).

\section{Discussion}

With a push toward personalized medicine, knowledge of the best time to perform RE around dinner will provide health care professionals with a better idea of how to personalize RE prescription to optimize its metabolic health benefits. Thus, the overarching goal of this project was to identify the best time, either before or after dinner, to perform RE to lower postprandial glucose and TAG concentrations, two important cardiovascular disease risk factors in obese patients with type- 2 diabetes. The key findings of this study are: 1) Both pre- and post-dinner RE reduces postprandial glucose concentrations, while only post-dinner RE reduces both postprandial glucose and TAG concentrations, 2) Both pre- and post-dinner RE reduces insulin concentrations, but via different mechanisms as pre-dinner RE enhances insulin clearance, whereas post-dinner RE reduces pancreatic insulin secretion and enhances insulin clearance, 3) Post-dinner RE reduces postprandial GLP-1 concentrations while pre-dinner RE does not, 4) Predinner RE reduces pre-dinner FFA concentrations and attenuates the decline in postprandial FFA concentrations, 5) Gastric emptying, beta-cell function, postprandial substrate oxidation, GIP concentrations, nocturnal and postprandial glucose concentrations or insulin sensitivity the following morning are not altered by pre- or postdinner RE, and 6) RE improved well-being when performed before or after dinner. Taken together, post-dinner RE improves postprandial metabolic risk factors better than predinner RE in obese patients with type-2 diabetes and these benefits are short lived and do 
not last into the overnight period or into the next day. Importantly, RE improved wellbeing, making it a feasible option in this population.

Previous research is mixed as two studies have shown that acute RE prior to a meal (s) improves glycaemic control [19] [18], but two other studies showed acute RE does not improve glycaemic control in patients with type-2 diabetes $[24,25]$. In the current study both pre- and post-dinner RE glycaemic control compared to NoRE, but neither RE time was significantly better compared to the other at doing so. With predinner RE, postprandial glucose concentrations were lower from $\sim 1-3 \mathrm{~h}$ after the meal compared to NoRE. During post-dinner RE, glucose concentrations were lower during exercise from $\sim 45 \mathrm{~min}-1.5 \mathrm{~h}$ after the meal, and rebounded at the cessation of exercise to NoRE levels. These drastic differences in glucose responses suggest different glucose lowering mechanisms may be at play depending on RE timing. Previous research has shown acute RE increases skeletal muscle GLUT 4 protein (independent of insulin) up to $3.5 \mathrm{~h}$ after exercise [34] and insulin sensitivity up to $24 \mathrm{~h}$ post $\mathrm{RE}[35,36]$, therefore during the pre-dinner RE trial, insulin independent glucose uptake and insulin action could have been increased prior to the meal in the previously exercised skeletal muscle and the liver, thus increasing postprandial skeletal muscle glucose uptake and reducing hepatic glucose output. During the post-dinner RE trial, elevated insulin concentrations due to the meal as well as the skeletal muscle contractions probably worked synergistically to increase skeletal muscle blood glucose uptake, as has been shown with aerobic exercise $[9,37]$. Interestingly, once the post-dinner RE session was over, blood glucose concentrations rebounded and were similar to those during the NoRE trial. This rebound in glucose may have been due to a simultaneous reduction in skeletal muscle 
glucose uptake at the cessation of exercise and a transient increase in hepatic glucose output, which has been shown to occur with postprandial aerobic exercise [9]. The improvements in glycaemia in the current study were short-lived and did not extend into the nocturnal period or into the morning period the next day, which suggests that daily exercise is required to maintain improvements in glycaemia.

For the first time, this study shows that post-dinner RE lowers postprandial TAG concentrations, whereas pre-dinner RE does not. The available data with aerobic exercise is similar as two studies have shown prior aerobic exercise performed the day prior to the test meal does not alter postprandial TAG concentrations [21,23], while in another study post-meal aerobic exercise reduced postprandial TAG concentrations compared to no exercise [20]. The majority of TAG in circulation originates from either exogenous sources and is in the form of chylomicron particles or endogenous sources from the liver and is in the form of VLDL-TAG [38]. We attempted to establish whether chylomicrons or VLDL-TAG were modified by RE timing, and observed that neither pre-meal nor post-meal RE modified postprandial chylomicron or VLDL-2 TAG particles. Instead, the reduction in postprandial TAG concentrations with post-dinner RE was mediated by reduced VLDL-1 TAG concentrations. Although not possible to determine from the data, the mechanism(s) for the reduction in VLDL-1 TAG with RE could have been mediated by enhanced hydrolysis of TAG by lipoprotein lipase in skeletal muscle and/or by reduced hepatic VLDL-1 TAG secretion [38].

In the current study, both pre- and post-dinner RE reduced postprandial insulin concentrations, a finding that is in agreement with other work [24] but with differing physiological mechanisms. With pre-dinner RE, the reduction in postprandial insulin 
concentrations was mediated by enhanced insulin clearance, and this has been shown before [24]. Although pre-dinner RE reduced glucose concentrations, this was not associated with reduced insulin secretion, possibly because the insulin potentiating hormones GLP-1 or GIP were not reduced. This finding is similar to another study in which pre-meal aerobic exercise did not alter postprandial GLP-1 or GIP responses in type 2 diabetic patients [39]. On the contrary, during post-dinner RE the reduction in insulin concentrations was mediated by both increased insulin clearance and reduced insulin secretion, although the increase in insulin clearance was not as great as compared to the pre-dinner RE trial. Lower postprandial insulin secretion with post-dinner RE could have been mediated by reduced GLP-1 and glucose concentrations, and not by changes in GIP or beta-cell function. The mechanism by which RE increases insulin clearance could potentially be due to increased skeletal muscle and/or liver blood flow. Increased blood flow in these tissues may increase blood flow through previously nonflowing capillaries, allowing more insulin to be exposed to and bind to the insulin receptor, internalized, and degraded. This hypothesis is supported by the work of Philips et al. [40] who showed that prior RE enhanced postprandial leg blood flow responses, compared to no exercise. Another possibility may by that RE reduced portal vein glucose and/or FFA concentrations. Given that glucose and FFA have been shown to independently and synergistically impair insulin binding to receptors [41], it is possible that a RE induced reduction in these substrates in the portal vein allowed more hepatic insulin clearance.

In conclusion, acute post-dinner RE improves postprandial metabolic risk factors better than pre-dinner RE in obese patients with type- 2 diabetes. Future studies are 
needed to determine if long-term post-meal exercise training would better reduce cardiovascular disease more so than pre-meal exercise.

\section{Acknowledgement}

We thank Ying Liu for placing the catheters and helping with some of the data collection.

This project was supported by department funds and a NIH 5T32AR048523-10 training grant.

\section{Contribution Statement}

T.D.H. Study conception, study design, data collection, data analysis, data interpretation, wrote manuscript.

N.C.W. Data collection, edited manuscript.

A.M. Beta cell function analysis, edited manuscript.

F.W.B. Study design, data interpretation, edited manuscript.

R.S.R. Study design, data interpretation, edited manuscript.

J.P.T. Study design, data interpretation, edited manuscript.

J.A.K. Study conception, study design, data collection, data analysis, data interpretation, edited manuscript. 


\section{References}

[1] Temelkova-Kurktschiev TS, Koehler C, Leonhardt W, et al. (1999) Increased intimal-medial thickness in newly detected type 2 diabetes: risk factors. Diabetes Care 22: $333-338$

[2] Hu Y, Liu W, Huang R, Zhang X (2010) Postchallenge plasma glucose excursions, carotid intima-media thickness, and risk factors for atherosclerosis in Chinese population with type 2 diabetes. Atherosclerosis 210: 302-306

[3] Temelkova-Kurktschiev TS, Koehler C, Henkel E, Leonhardt W, Fuecker K, Hanefeld M (2000) Postchallenge plasma glucose and glycemic spikes are more strongly associated with atherosclerosis than fasting glucose or HbAlc level. Diabetes Care 23: $1830-1834$

[4] Teno S, Uto Y, Nagashima H, et al. (2000) Association of postprandial hypertriglyceridemia and carotid intima-media thickness in patients with type 2 diabetes. Diabetes Care 23: 1401-1406

[5] Nordestgaard BG, Benn M, Schnohr P, Tybjaerg-Hansen A (2007) Nonfasting triglycerides and risk of myocardial infarction, ischemic heart disease, and death in men and women. JAMA 298: 299-308

[6] Freiberg JJ, Tybjaerg-Hansen A, Jensen JS, Nordestgaard BG (2008) Nonfasting triglycerides and risk of ischemic stroke in the general population. JAMA 300: 21422152

[7] O'Gorman DJ, Krook A (2011) Exercise and the treatment of diabetes and obesity. Med Clin North Am 95: 953-969

[8] Krook A, Holm I, Pettersson S, Wallberg-Henriksson H (2003) Reduction of risk factors following lifestyle modification programme in subjects with type 2 (non-insulin dependent) diabetes mellitus. Clinical physiology and functional imaging 23: 21-30

[9] Larsen JJ, Dela F, Kjaer M, Galbo H (1997) The effect of moderate exercise on postprandial glucose homeostasis in NIDDM patients. Diabetologia 40: 447-453

[10] Larsen JJ, Dela F, Madsbad S, Galbo H (1999) The effect of intense exercise on postprandial glucose homeostasis in type II diabetic patients. Diabetologia 42: 1282-1292

[11] Praet SF, Manders RJ, Lieverse AG, et al. (2006) Influence of acute exercise on hyperglycemia in insulin-treated type 2 diabetes. Med Sci Sports Exerc 38: 2037-2044

[12] Manders RJ, Van Dijk JW, van Loon LJ (2010) Low-intensity exercise reduces the prevalence of hyperglycemia in type 2 diabetes. Med Sci Sports Exerc 42: 219-225

[13] Van Dijk JW, Manders RJ, Canfora EE, et al. (2013) Exercise and 24-h glycemic control: equal effects for all type 2 diabetes patients? Med Sci Sports Exerc 45: 628-635

[14] van Dijk JW, Tummers K, Stehouwer CD, Hartgens F, van Loon LJ (2012) Exercise therapy in type 2 diabetes: is daily exercise required to optimize glycemic control? Diabetes Care 35: 948-954

[15] van Dijk JW, Venema M, van Mechelen W, Stehouwer CD, Hartgens F, van Loon LJ (2013) Effect of moderate-intensity exercise versus activities of daily living on 24hour blood glucose homeostasis in male patients with type 2 diabetes. Diabetes Care 36 : 3448-3453

[16] Gillen JB, Little JP, Punthakee Z, Tarnopolsky MA, Riddell MC, Gibala MJ (2012) Acute high-intensity interval exercise reduces the postprandial glucose response and 
prevalence of hyperglycaemia in patients with type 2 diabetes. Diabetes Obes Metab 14: 575-577

[17] Oberlin DJ, Mikus CR, Kearney ML, et al. (2014) One bout of exercise alters freeliving postprandial glycemia in type 2 diabetes. Med Sci Sports Exerc 46: 232-238

[18] van Dijk JW, Manders RJ, Tummers K, et al. (2012) Both resistance- and endurance-type exercise reduce the prevalence of hyperglycaemia in individuals with impaired glucose tolerance and in insulin-treated and non-insulin-treated type 2 diabetic patients. Diabetologia 55: 1273-1282

[19] Fenicchia LM, Kanaley JA, Azevedo JL, Jr., et al. (2004) Influence of resistance exercise training on glucose control in women with type 2 diabetes. Metabolism 53: 284289

[20] Tobin LW, Kiens B, Galbo H (2008) The effect of exercise on postprandial lipidemia in type 2 diabetic patients. Eur J Appl Physiol 102: 361-370

[21] Gill JM, Al-Mamari A, Ferrell WR, et al. (2007) Effect of prior moderate exercise on postprandial metabolism in men with type 2 diabetes: heterogeneity of responses. Atherosclerosis 194: 134-143

[22] Colberg SR, Zarrabi L, Bennington L, et al. (2009) Postprandial walking is better for lowering the glycemic effect of dinner than pre-dinner exercise in type 2 diabetic individuals. J Am Med Dir Assoc 10: 394-397

[23] Dalgaard M, Thomsen C, Hermansen K (2004) Effects of one single bout of lowintensity exercise on postprandial lipaemia in type 2 diabetic men. Br J Nutr 92: 469-476 [24] Fluckey JD, Hickey MS, Brambrink JK, Hart KK, Alexander K, Craig BW (1994) Effects of resistance exercise on glucose tolerance in normal and glucose-intolerant subjects. J Appl Physiol (1985) 77: 1087-1092

[25] Bacchi E, Negri C, Trombetta M, et al. (2012) Differences in the acute effects of aerobic and resistance exercise in subjects with type 2 diabetes: results from the RAED2 Randomized Trial. PloS one 7: e49937

[26] Garg A, Bantle JP, Henry RR, et al. (1994) Effects of varying carbohydrate content of diet in patients with non-insulin-dependent diabetes mellitus. JAMA 271: 1421-1428 [27] Abbasi F, Chu JW, McLaughlin T, Lamendola C, Leary ET, Reaven GM (2004) Effect of metformin treatment on multiple cardiovascular disease risk factors in patients with type 2 diabetes mellitus. Metabolism 53: 159-164

[28] Karpe F, Hamsten A (1994) Determination of apolipoproteins B-48 and B-100 in triglyceride-rich lipoproteins by analytical SDS-PAGE. J Lipid Res 35: 1311-1317

[29] Mari A, Schmitz O, Gastaldelli A, Oestergaard T, Nyholm B, Ferrannini E (2002) Meal and oral glucose tests for assessment of beta -cell function: modeling analysis in normal subjects. Am J Physiol Endocrinol Metab 283: E1159-1166

[30] Mari A, Tura A, Gastaldelli A, Ferrannini E (2002) Assessing insulin secretion by modeling in multiple-meal tests: role of potentiation. Diabetes 51 Suppl 1: S221-226

[31] Van Cauter E, Mestrez F, Sturis J, Polonsky KS (1992) Estimation of insulin secretion rates from $\mathrm{C}$-peptide levels. Comparison of individual and standard kinetic parameters for C-peptide clearance. Diabetes 41: 368-377

[32] Pruessner JC, Kirschbaum C, Meinlschmid G, Hellhammer DH (2003) Two formulas for computation of the area under the curve represent measures of total hormone concentration versus time-dependent change. Psychoneuroendocrinology 28: 916-931 
[33] McDonnell CM, Donath SM, Vidmar SI, Werther GA, Cameron FJ (2005) A novel approach to continuous glucose analysis utilizing glycemic variation. Diabetes technology \& therapeutics 7: 253-263

[34] Gallagher PM, Touchberry CD, Teson K, McCabe E, Tehel M, Wacker MJ (2013) Effects of an acute bout of resistance exercise on fiber-type specific to GLUT4 and IGF1R expression. Appl Physiol Nutr Metab 38: 581-586

[35] Reed ME, Ben-Ezra V, Biggerstaff KD, Nichols DL (2012) The effects of two bouts of high- and low-volume resistance exercise on glucose tolerance in normoglycemic women. J Strength Cond Res 26: 251-260

[36] Black LE, Swan PD, Alvar BA (2010) Effects of intensity and volume on insulin sensitivity during acute bouts of resistance training. J Strength Cond Res 24: 1109-1116

[37] Thorell A, Hirshman MF, Nygren J, et al. (1999) Exercise and insulin cause GLUT4 translocation in human skeletal muscle. Am J Physiol 277: E733-741

[38] Ginsberg HN, Zhang YL, Hernandez-Ono A (2005) Regulation of plasma triglycerides in insulin resistance and diabetes. Arch Med Res 36: 232-240

[39] Eshghi SR, Bell GJ, Boule NG (2013) Effects of Aerobic Exercise with or without Metformin on Plasma Incretins in Type 2 Diabetes. Can J Diabetes 37: 375-380

[40] Phillips B, Williams J, Atherton P, et al. (2012) Resistance exercise training improves age-related declines in leg vascular conductance and rejuvenates acute leg blood flow responses to feeding and exercise. J Appl Physiol (1985) 112: 347-353

[41] Hennes MM, Dua A, Kissebah AH (1997) Effects of free fatty acids and glucose on splanchnic insulin dynamics. Diabetes 46: 57-62

\section{Tables}

Table 1. Patient Characteristics ( $\mathrm{N}=13,5$ Males)

\begin{tabular}{lc} 
Age $(\mathrm{yr})$ & $48.5 \pm 11.9$ \\
Height $(\mathrm{m})$ & $1.67 \pm 0.11$ \\
Weight $(\mathrm{kg})$ & $103.2 \pm 22.8$ \\
Body mass index $\left(\mathrm{kg} / \mathrm{m}^{2}\right)$ & $36.7 \pm 5.3$ \\
Body fat percent & $39.5 \pm 8.6$ \\
Fasting Glucose $(\mathrm{mmol} / \mathrm{L})$ & $8.2 \pm 2.3$ \\
Fasting Hemoglobin A1c $(\%$ [mmol/mol] $)$ & $7.2 \pm 1.1[55]$ \\
Diagnosed with Type 2 Diabetes $(\mathrm{yr})$ & $3.7 \pm 3.9$ \\
Patients with antidiabetes medication use, $\mathrm{n}$ & 12 \\
\hline
\end{tabular}

Values are means \pm SD 
Table 2. Metabolic data and rating of perceived exertion during resistance exercise session.

\begin{tabular}{lcccc}
\hline & $\mathrm{RE} \rightarrow \mathrm{M}$ & NoRE- 1 & $\mathrm{M} \rightarrow \mathrm{RE}$ & NoRE-1 \\
\hline Time (min) & $46 \pm 1$ & $46 \pm 1$ & $47 \pm 1$ & $47 \pm 1$ \\
Oxygen consumption $\left(\mathrm{ml} \mathrm{kg}^{-1} \mathrm{~min}^{-1}\right)$ & $6.1 \pm 0.4$ & $2.9 \pm 0.1^{*}$ & $6.0 \pm 0.3$ & $2.9 \pm 0.1^{*}$ \\
Energy Expenditure (Gross kj) & $586 \pm 59$ & $264 \pm 13^{*}$ & $590 \pm 59$ & $276 \pm 21^{*}$ \\
Respiratory Exchange Ratio & $1.00 \pm 0.01$ & $0.83 \pm 0.01^{*}$ & $1.00 \pm 0.01$ & $0.87 \pm 0.01^{* \dagger}$ \\
Heart Rate (bpm) & $106 \pm 4$ & & $110 \pm 4^{* *}$ & \\
Average RPE (BORG 6-20 scale) & $12 \pm 1$ & & $12 \pm 1$ & \\
\hline
\end{tabular}

Values are means \pm S.E.M.

The data under NoRE-1 is during the same time frame (i.e. before dinner) as $\mathrm{RE} \rightarrow \mathrm{M}$ but during the NoRE trial, while the data under NoRE-2 is during the same time frame (i.e. after dinner) as $\mathrm{M} \rightarrow \mathrm{RE}$ but during the NoRE trial.

$* \mathrm{P}<0.05$ compared to $\mathrm{RE} \rightarrow \mathrm{M}$ or $\mathrm{M} \rightarrow \mathrm{RE}$.

$* * \mathrm{P}<0.05$ compared to heart rate during the $\mathrm{RE} \rightarrow \mathrm{M}$ trial.

$\dagger \mathrm{P}<0.05$ compared to NoRE-1.

For the variables time, oxygen consumption, energy expenditure, respiratory exchange ratio, and heart rate, only data for $\mathrm{N}=12$ is reported. For $\mathrm{RPE}$, data for $\mathrm{N}$ $=13$ is reported.

Table 3. Energy Expenditure and Substrate Oxidation $\sim 3.5$ - 4 h After Dinner Meal

\begin{tabular}{lcc}
\hline & Energy Expenditure $\left(\mathrm{kj} \mathrm{min}^{-1}\right)$ & RER \\
\hline NoRE & $5.9 \pm 0.4$ & $0.85 \pm 0.01$ \\
$\mathrm{RE} \rightarrow \mathrm{M}$ & $5.9 \pm 0.4$ & $0.83 \pm 0.01$ \\
$\mathrm{M} \rightarrow \mathrm{RE}$ & $6.3 \pm 0.4$ & $0.83 \pm 0.01$ \\
\hline
\end{tabular}

Values are means \pm S.E.M. 
Table 4. Beta-cell Function Parameters and Gastric Emptying

\begin{tabular}{lccc}
\hline & NoRE & $\mathrm{RE} \rightarrow \mathrm{M}$ & $\mathrm{M} \rightarrow \mathrm{RE}$ \\
\hline $\begin{array}{l}\text { Beta-cell glucose } \\
\text { sensitivity }\left(\left(\mathrm{pmol} /\left(\mathrm{min} \mathrm{m}^{2}\right.\right.\right.\end{array}$ & $39 \pm 5$ & $50 \pm 8$ & $50 \pm 9$ \\
$\mathrm{mM})$ ) & $306 \pm 93$ & $402 \pm 141$ & $307 \pm 86$ \\
$\begin{array}{l}\text { Rate Sensitivity } \\
\left(\mathrm{pmol} /\left(\mathrm{m}^{2} \mathrm{mM}\right)\right)\end{array}$ & $1.28 \pm 0.14$ & $0.99 \pm 0.08$ & $0.92 \pm 0.10$ \\
$\begin{array}{l}\text { Potentiation Factor Ratio } \\
\text { (fold) }\end{array}$ & $17,777 \pm 1,866$ & $15,493 \pm 1,792$ & $19,001 \pm 1,626$ \\
$\begin{array}{l}\text { Acetaminophen } \text { iAUC } \\
(\mu \mathrm{mol} / \mathrm{L})\end{array}$ & & & \\
\hline Values are means \pm S.E.M. & & &
\end{tabular}

Table 5. Average Blood Glucose and Glycemic Variability Over $24 \mathrm{~h}$

\begin{tabular}{lccc}
\hline & NoRE & RE-M & M-RE \\
\hline $\begin{array}{l}\text { Average Blood Glucose } \\
\text { (mmol/L) }\end{array}$ & $7.8 \pm 0.4$ & $7.2 \pm 0.4$ & $7.8 \pm 0.6$ \\
Standard Deviation & $1.8 \pm 0.2$ & $1.7 \pm 0.2$ & $2.1 \pm 0.2$ \\
CONGA-1 & $6.8 \pm 0.4$ & $6.3 \pm 0.3$ & $6.7 \pm 0.5$ \\
CONGA-2 & $6.1 \pm 0.4$ & $5.7 \pm 0.3$ & $6.0 \pm 0.5$ \\
CONGA-4 & $5.7 \pm 0.4$ & $5.5 \pm 0.3$ & $5.4 \pm 0.5$ \\
\hline
\end{tabular}

Values are means \pm S.E.M.

Data is for $\mathrm{N}=11$ participants as 2 participants had missing data so they were excluded.

Table 6. Nocturnal and Post-Breakfast Glucose Responses

\begin{tabular}{|c|c|c|c|}
\hline & NoRE & $\mathrm{RE} \rightarrow \mathrm{M}$ & $\mathrm{M} \rightarrow \mathrm{RE}$ \\
\hline \multicolumn{4}{|l|}{ Nocturnal Glucose Responses (12-6 a.m.) } \\
\hline Average Blood Glucose (mmol/L) & $6.3 \pm 0.5$ & $5.9 \pm 0.4$ & $6.4 \pm 0.6$ \\
\hline $\begin{array}{l}\text { Time Hyperglycemic }(\mathrm{min}) \\
\text { (Blood Glucose }>10 \mathrm{mmol} / \mathrm{L})\end{array}$ & $0.4 \pm 0.6$ & $2.7 \pm 3.9$ & $14.6 \pm 10.6$ \\
\hline $\begin{array}{l}\text { Time Hypoglycemic }(\mathrm{min}) \\
\text { (Blood Glucose }<3.9 \mathrm{mmol} / \mathrm{L})\end{array}$ & $26.5 \pm 30.5$ & $17.7 \pm 7.8$ & $6.9 \pm 3.7$ \\
\hline \multicolumn{4}{|l|}{ Morning Glucose Responses } \\
\hline $\begin{array}{r}\text { Average } \\
(\mathrm{mmol} / \mathrm{L})\end{array}$ & $8.9 \pm 0.6$ & $8.7 \pm 0.7$ & $9.1 \pm 0.9$ \\
\hline Breakfast $4 \mathrm{~h}$ iAUC (mmol/L per $4 \mathrm{~h})$ & $72 \pm 17$ & $83 \pm 23$ & $75 \pm 22$ \\
\hline $\begin{array}{l}\text { Time Hyperglycemic }(\mathrm{min}) \\
(\text { Blood Glucose }>10 \mathrm{mg} / \mathrm{dL})\end{array}$ & $80.8 \pm 22.7$ & $43.3 \pm 19.0$ & $66.3 \pm 27.5$ \\
\hline $\begin{array}{l}\text { Time Hypoglycemic }(\mathrm{min}) \\
(\text { Blood Glucose }<3.9 \mathrm{mg} / \mathrm{dL})\end{array}$ & 0 & 0 & 0 \\
\hline
\end{tabular}

Values are means \pm S.E.M. 
Table 7. Morning Fasting Measures The Day After Testing In The Lab

\begin{tabular}{lccc}
\hline & NoRE & $\mathrm{RE} \rightarrow \mathrm{M}$ & $\mathrm{M} \rightarrow \mathrm{RE}$ \\
\hline Blood Glucose (mmol/L) & $6.7 \pm 0.4$ & $6.7 \pm 0.3$ & $6.7 \pm 0.6$ \\
Insulin (pmol/L) & $290 \pm 43$ & $256 \pm 37$ & $301 \pm 53$ \\
C-Peptide (pmol/L) & $1537 \pm 181$ & $1378 \pm 113$ & $1465 \pm 194$ \\
Insulin Clearance (\%) & $81 \pm 2$ & $82 \pm 2$ & $80 \pm 2$ \\
HOMA-IR & $12.58 \pm 1.98$ & $10.98 \pm 1.77$ & $12.05 \pm 1.92$ \\
QUICKI & $0.28 \pm 0.01$ & $0.28 \pm 0.01$ & $0.28 \pm 0.05$ \\
Triacylglycerol (mmol/L) & $1.5 \pm 0.2$ & $1.5 \pm 0.2$ & $1.6 \pm 0.2$ \\
\hline Values are means \pm S.E.M. & & & \\
\hline
\end{tabular}

The data for the nocturnal glucose response is for $\mathrm{N}=13$ while the data for the morning glucose responses is for $\mathrm{N}=12$.

\section{Figures}

A. Glucose Time Course

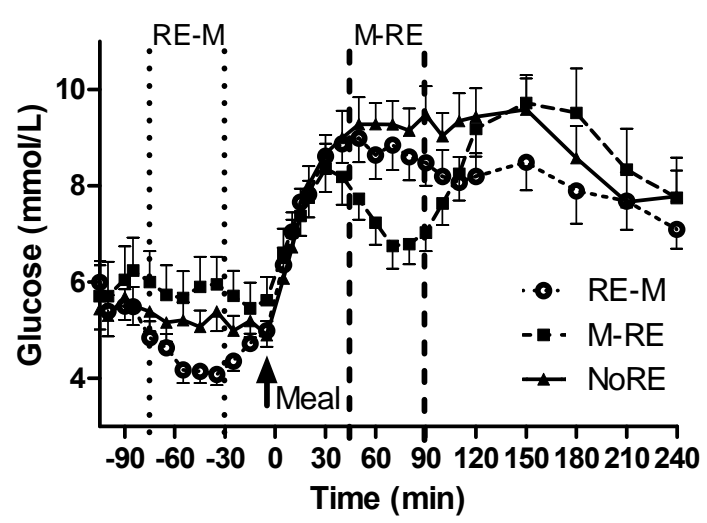

B. Pre-meal Glucose iAUC

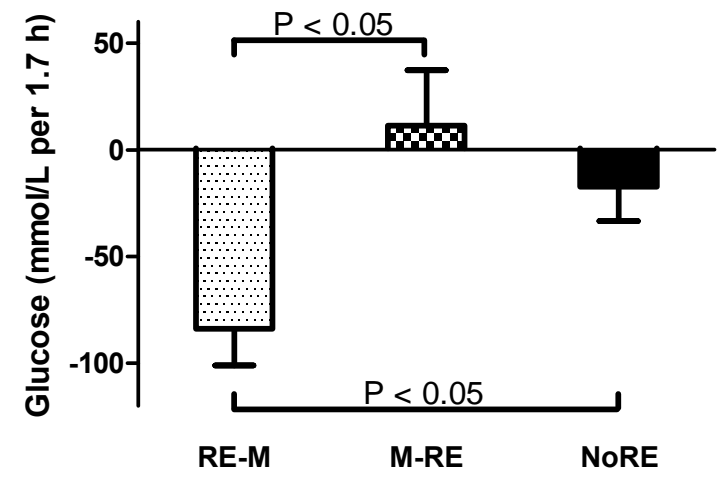

C. Post-meal Glucose iAUC
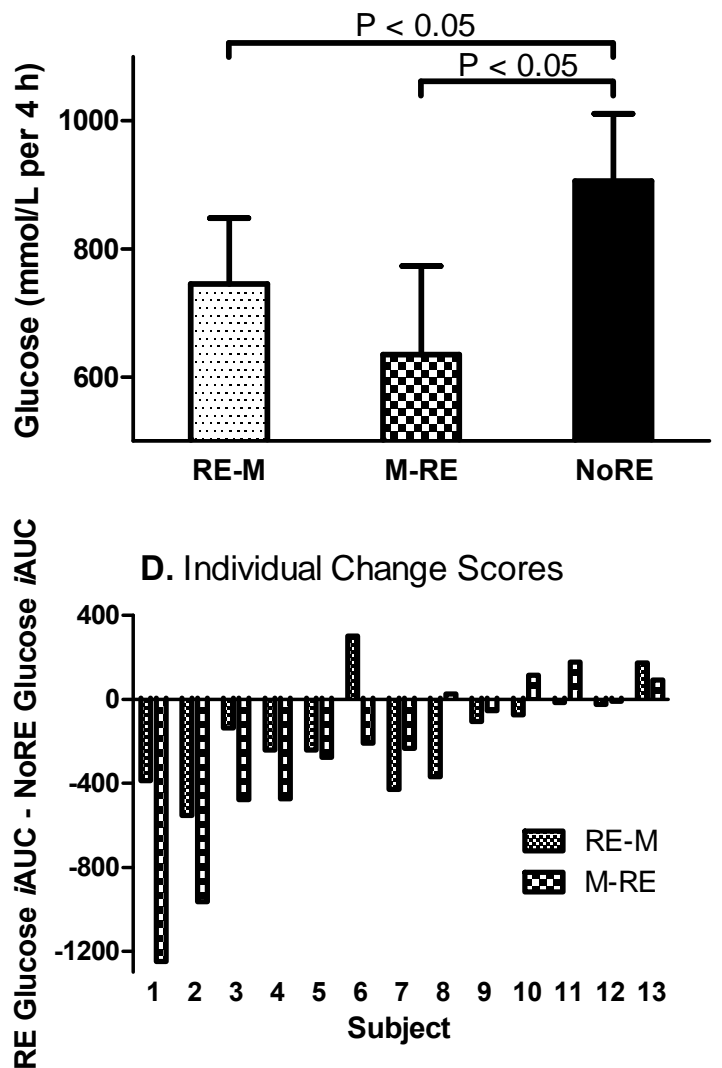

Figure 1. Glucose Responses During Testing 

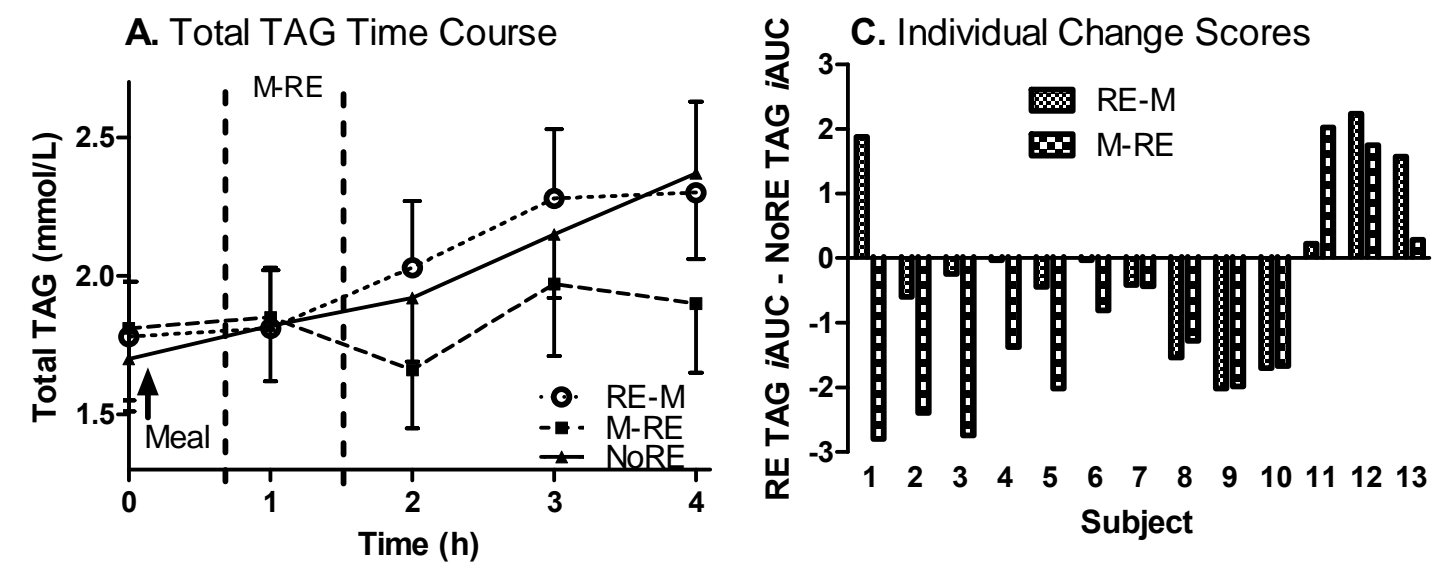

B. Total TAG iAUC

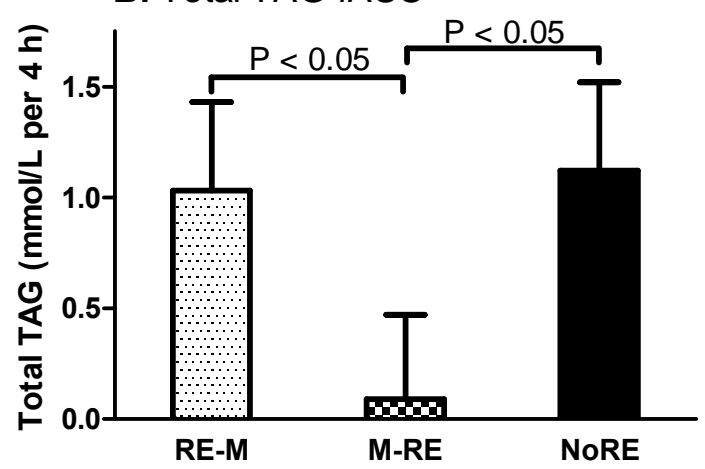

Figure 2. Total Triacylglycerol Responses During Testing 

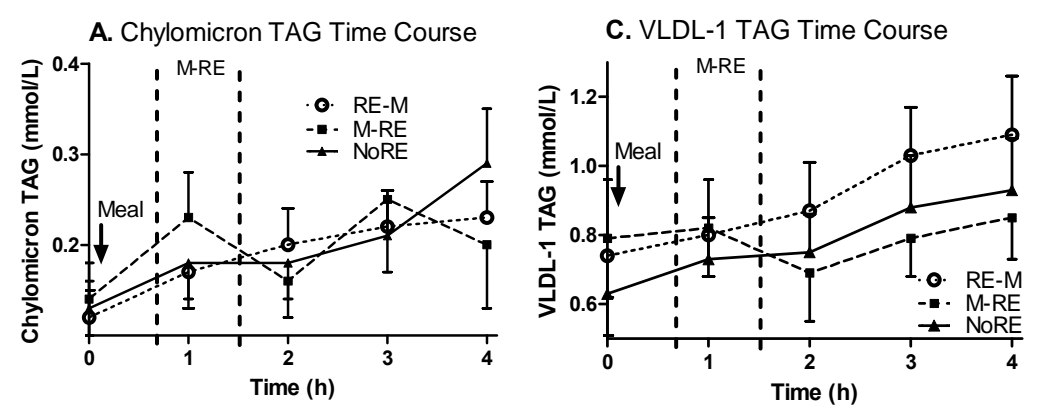

E. VLDL-2 TAG Time Course
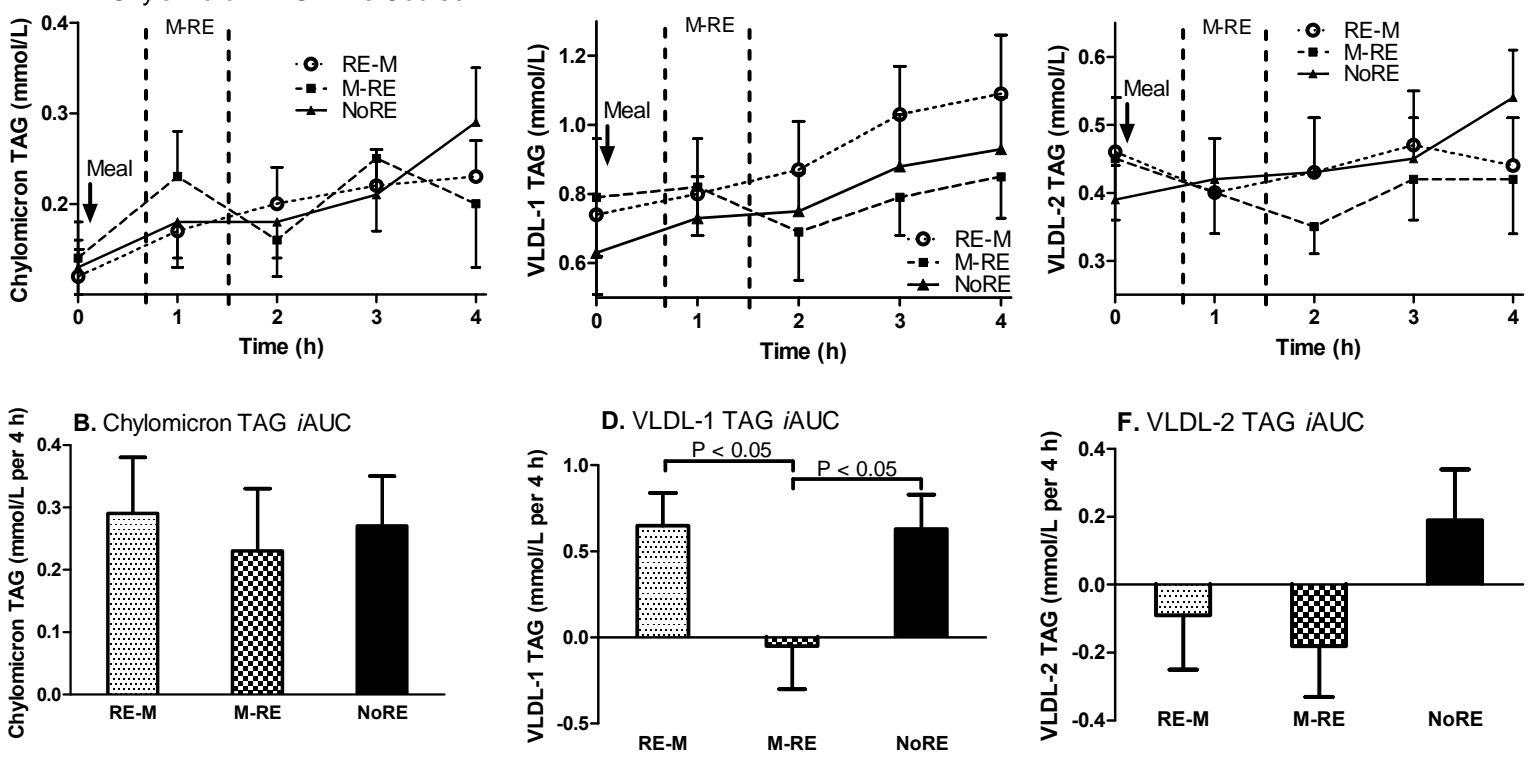

Figure 3. Exogenous and Endogenous Triacylglycerol Responses During Testing 
A. Free Fatty Acid Time Course

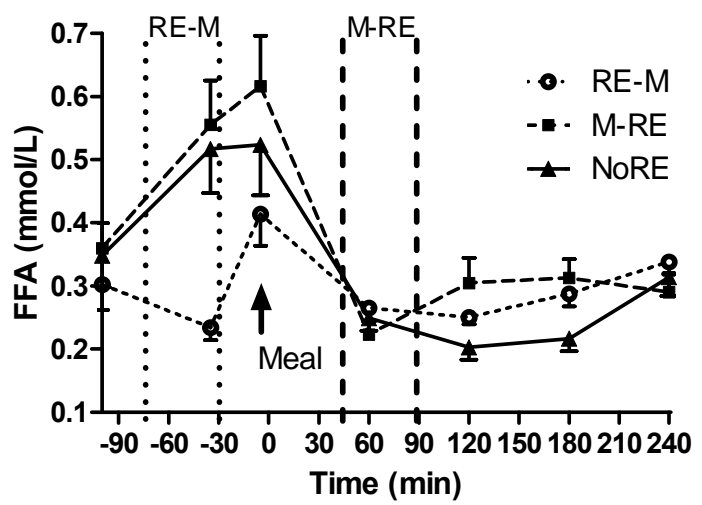

B. Pre-meal FFA iAUC

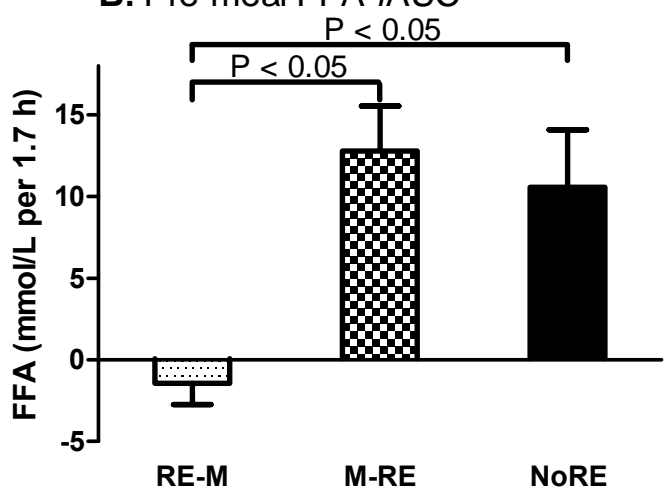

C. Post-meal FFA iAUC

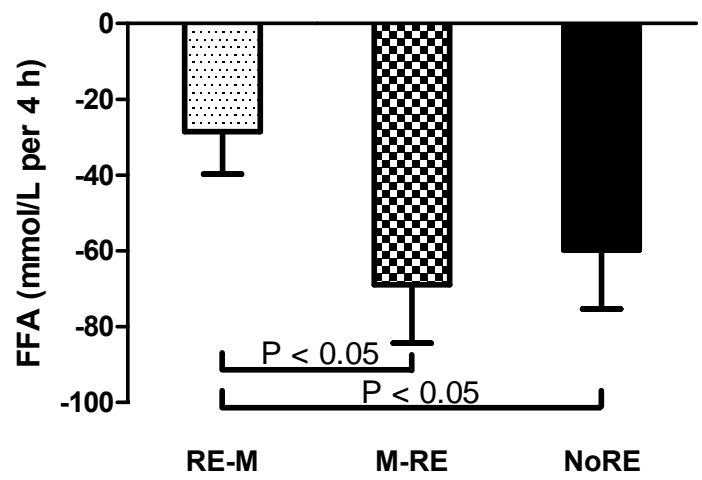

Figure 4. Free Fatty Acid Responses During Testing 
A. Insulin Time Course

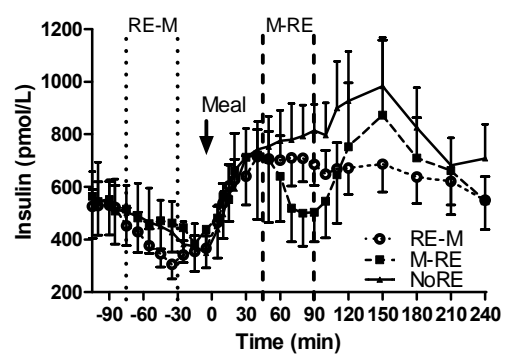

B. Pre-meal Insulin iAUC

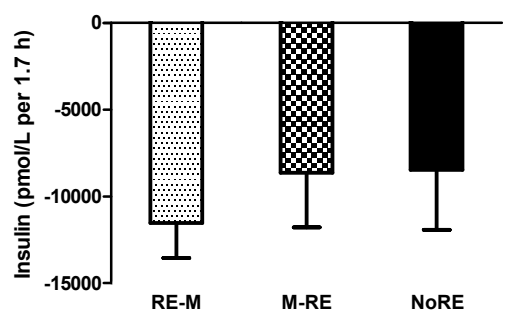

C. Post-meal Insulin iAUC

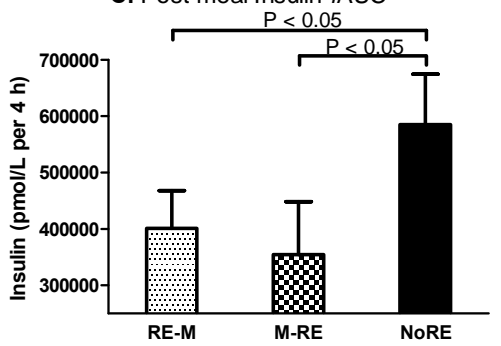

D. C-peptide Time Course

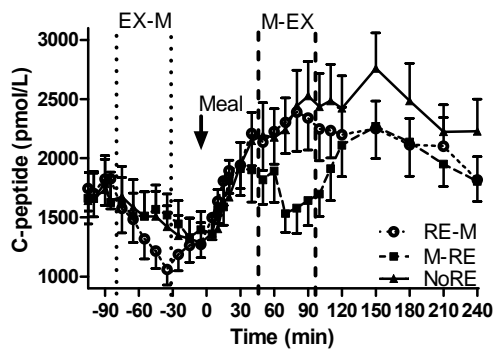

E. Pre-meal C-peptide iAUC

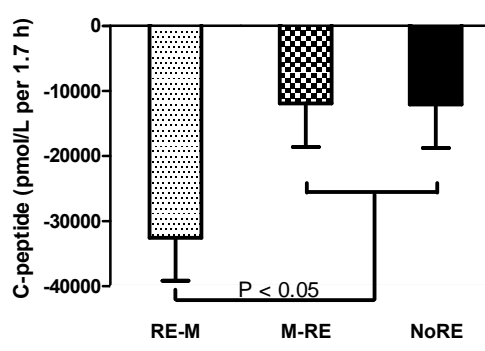

F. Post-meal C-peptide iAUC

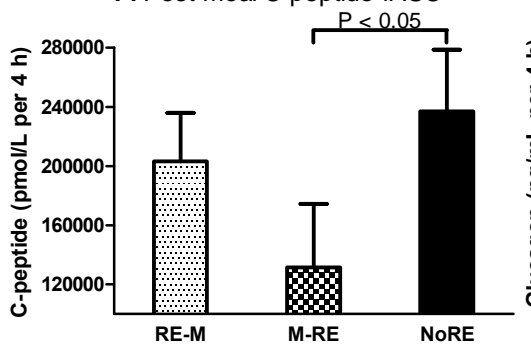

G. Glucagon Time Course

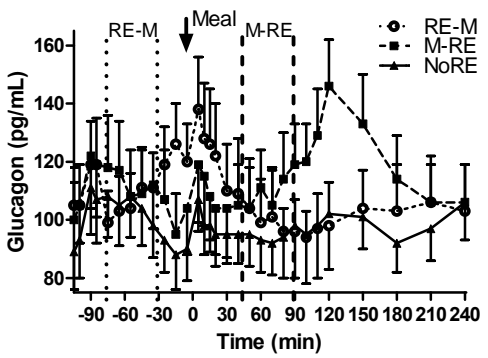

H. Pre-meal Glucagon iAUC

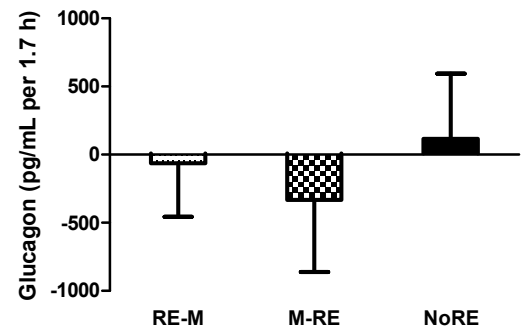

I. Post-meal Glucagon iAUC

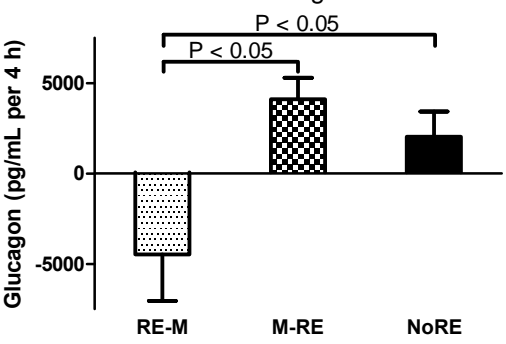

Figure 5. Pancreatic Hormone Responses During Testing 

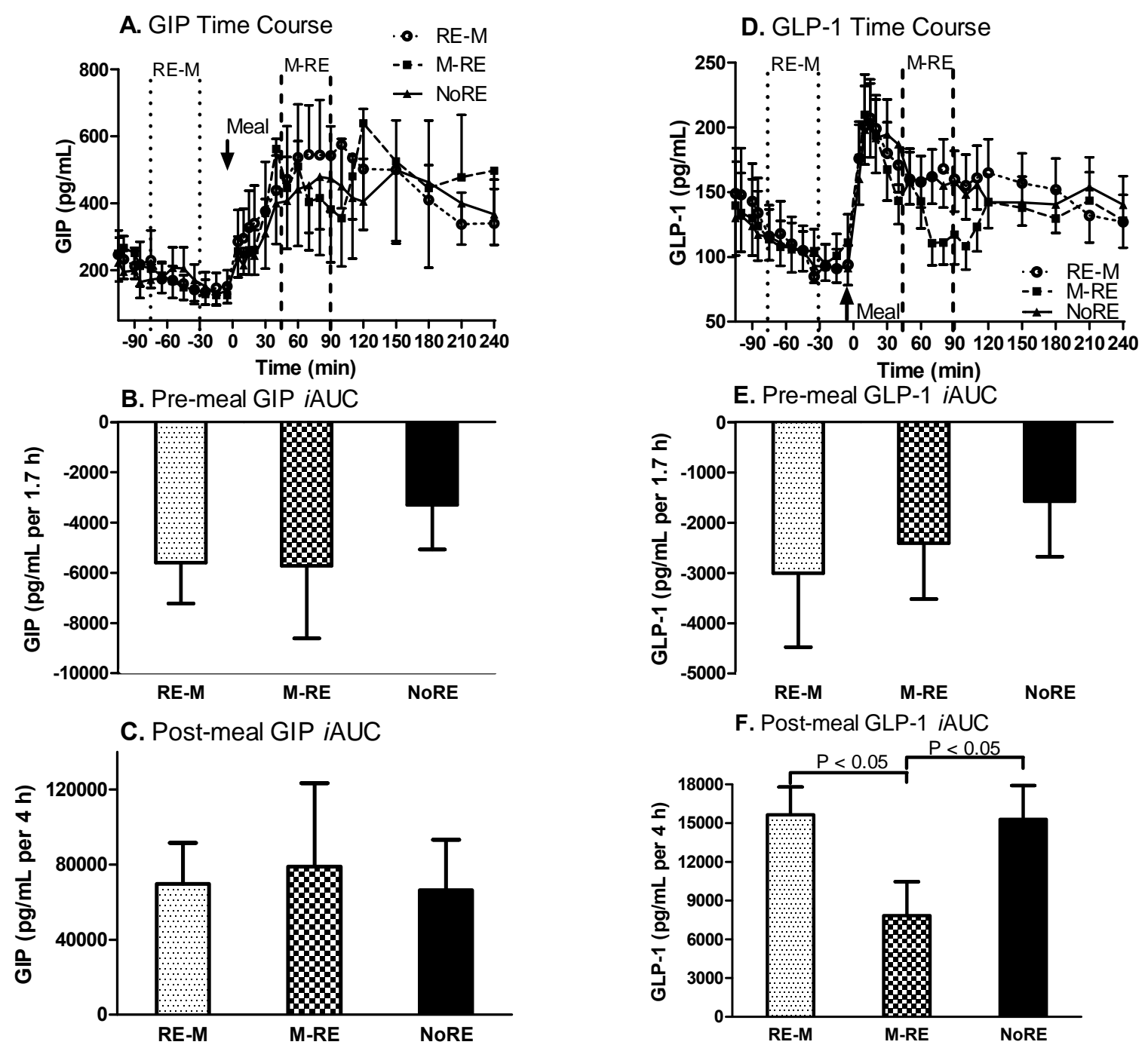

Figure 6. Gut Hormone Responses During Testing 
A. ISR Time Course

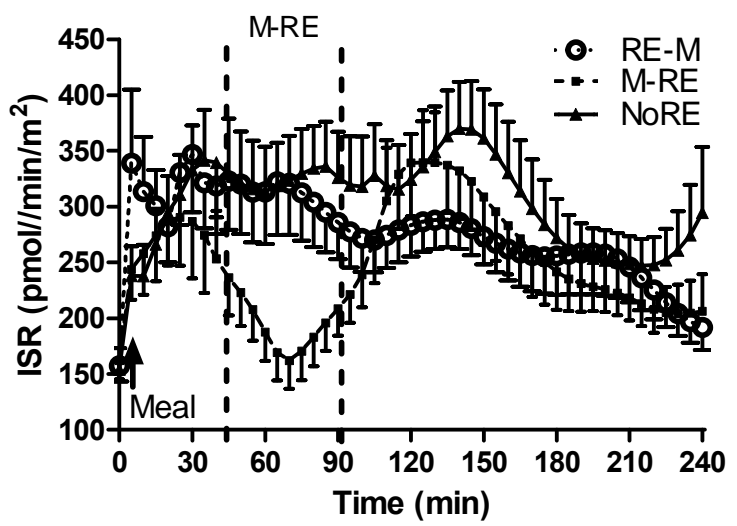

B. Post-meal ISR iAUC

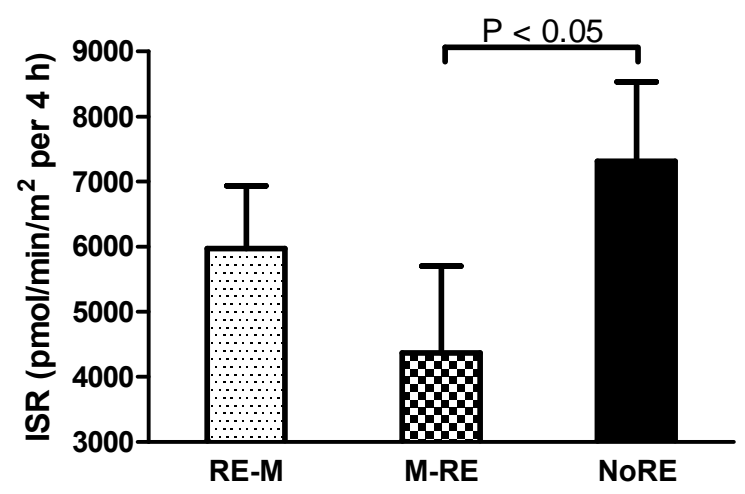

Figure 7. Insulin Kinetics During Testing
B. Insulin Clearance Time Course

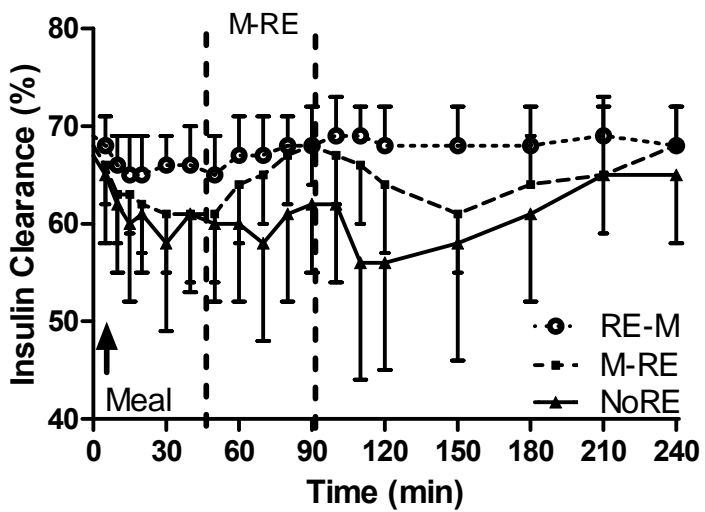

D. Post-meal Insulin Clearance iAUC

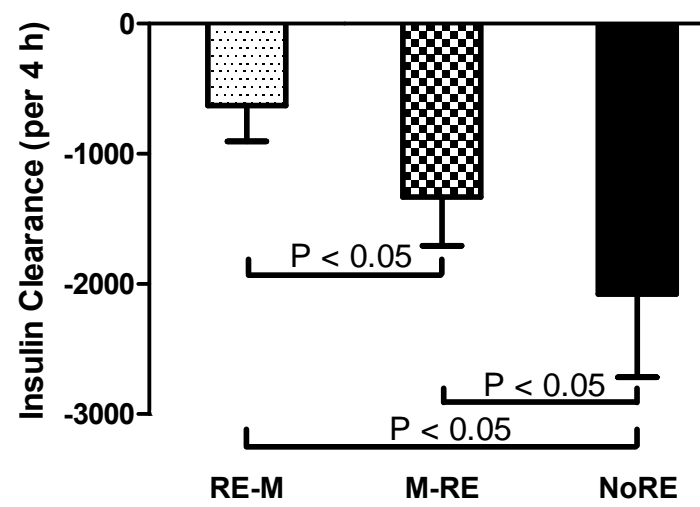


A. Pre-meal Change in Well-Being

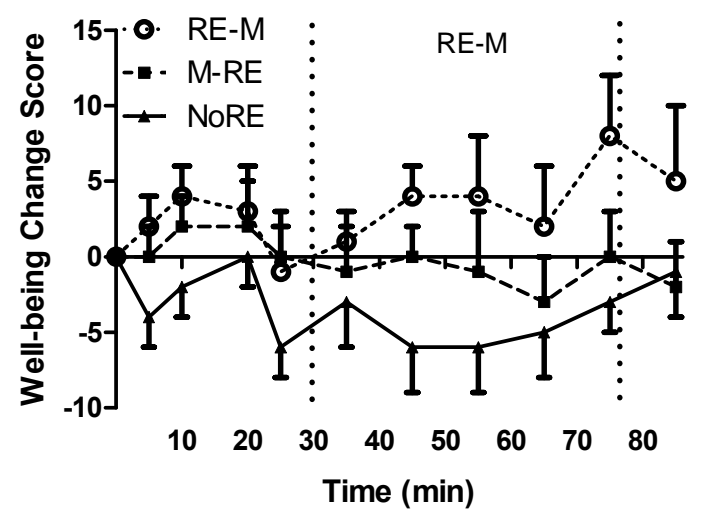

B. Pre-meal Well-being iAUC

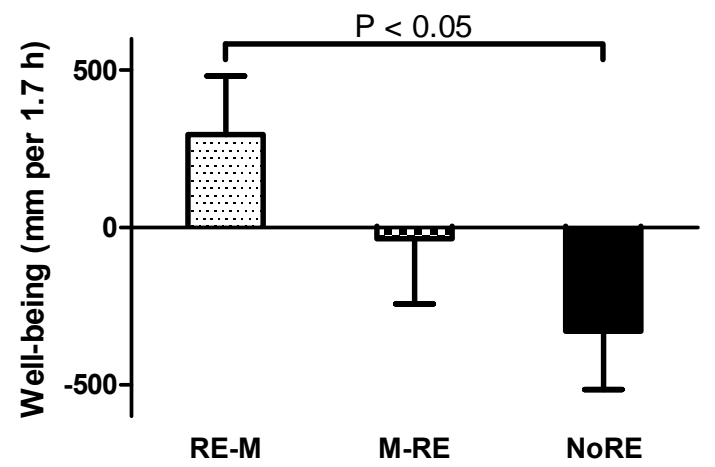

C. Post-meal Change in Well-Being

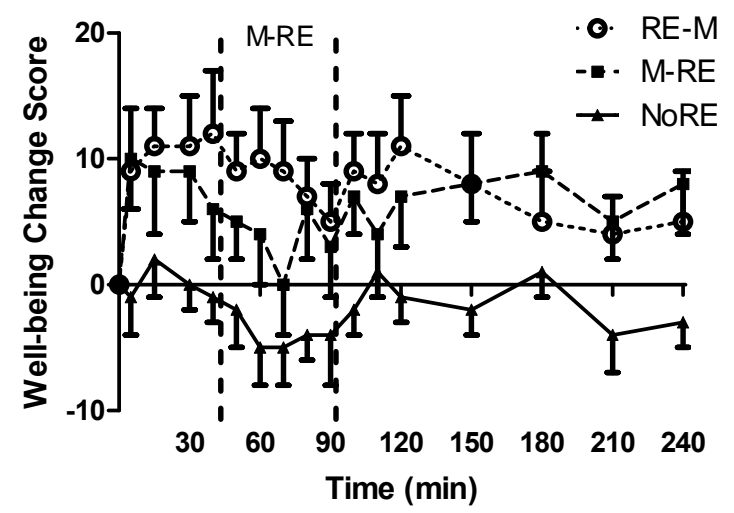

D. Post-meal Well-being iAUC

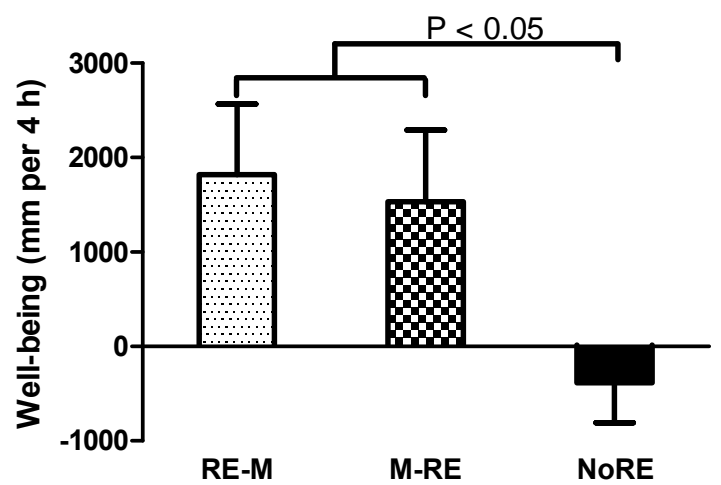

Figure 8. Subjective Well-being During Testing 
APPENDIX C

\title{
CURRICULUM VITAE
}

\author{
Timothy D. Heden \\ Ph.D. Candidate \\ Exercise Physiology \\ University of Missouri - Columbia \\ Phone: (573) 777-0499 \\ Email: tdheden@mail.mizzou.edu
}

\section{EDUCATION}

May 2007

July 2009

Pursuing
B.S. in Kinesiology, Southern Illinois University Edwardsville M.S. in Kinesiology, Southern Illinois University Edwardsville Ph.D. in Exercise Physiology, University of Missouri Columbia

\section{TRAINING EXPERIENCE}

\section{Training Grants}

2013-Present Trainee on NIH T32 Training Grant, Exercise and Health: Integration from Molecule to Patient (PI: Ron Terjung).

\section{Graduate Assistantships}

2009-2013 Research and Teaching Assistant, Department of Nutrition And Exercise Physiology, University of Missouri, Columbia, Missouri.

2007-2009 Research and Teaching Assistant, Kinesiology and Health Education Department, Southern Illinois University, Edwardsville, Illinois.

\section{CERTIFICATIONS}

2007-Present American College of Sports Medicine (ACSM) Certified Personal Trainer

2007-Present Adult CPR and AED

2008-Present National Strength and Conditioning Association (NSCA) Certified Strength and Conditioning Specialist

\section{HONORS AND AWARDS}

2013 MU M. Harold Laughlin Scholarship

2013 MU Ben R. Londeree / Tom R. Thomas Award in Exercise Physiology 
2012 Central States ACSM Doctoral Student Research Award

2012 MU Graduate Professional Council Travel Award

2012 MU HES Edward J. O’Brien Award in Nutritional Sciences

2011 MU Institute for Clinical and Translational Science Travel Award

2011 MU HES Seabaugh/Schade Fellowship in Nutritional Sciences

2010 MU HES Week $1^{\text {st }}$ Place Poster Award - Student Category

2009 MU Graduate Professional Council Travel Award

2009 Central States ACSM Best Poster Presentation Award

2009 SIUE Outstanding Kinesiology Graduate Student Award

2008 SIUE Outstanding Teaching Assistant

2008 SIUE Research Grants for Graduate Students Award

2007 SIUE Best Senior Project Award for Natural Science and Math

2006, 2007, and 2008 IAHPERD Student/Mentor Awards

2007 SIUE University Housing Student Employee of the Year Award

\section{PROFESSIONAL AFFILIATIONS}

2007-2009

2008-Present

2009-2012

2011-2012

2012-2013
Midwest American College of Sports Medicine (MW ACSM)

American College of Sports Medicine (ACSM)

Central States American College of Sports Medicine (CSACSM)

American Heart Association (AHA)

American Physiological Society (APS)

\section{PROFESSIONAL SERVICE}

\section{Journal Reviewer}

2009

2012-Present

2012-Present

2014

2014

2014
International Journal of Exercise Science

Medicine and Science in Sports and Exercise

Journal of Applied Physiology

Journal of Sports Medicine and Physical Fitness

Food and Function

International Journal of Chronic Obstructive Pulmonary Disease

\section{PEER-REVIWED PUBLICATIONS}

1) Heden TD, Liu Y, Park Y, Nyhoff LM, Winn NC, Kanaley JA. Moderate amounts of fructose- or glucose-sweetened beverages do not differentially alter metabolic health in male or female adolescents. Am J Clin Nutr. Accepted May 2014.

2) Kanaley JA, Heden TD, Liu Y, Fairchild TJ. Altering postprandial glucose and insulin concentrations with meal frequency and composition. Br J Nutr. Accepted June 2014. 
3) Heden TD, Morris EM, Kearney ML, Liu T, Park Y, Kanaley JA, Thyfault JP. Differential effects of low-fat and high-fat diets on fed state hepatic triacylglycerol secretion, hepatic fatty acid profiles, and DGAT-1 protein expression in obese prone Sprague-Dawley rats. App Physiol Nutr Metab. 2014;39(4):472-9.

4) Heden TD, Liu Y, Kearney ML, Kanaley JA. Weight classification does not influence the short-term endocrine and metabolic effects of high-fructose corn syrup sweetened beverages. App Physiol Nutr Metab. 2014;39(5):544-52

5) Heden TD, Liu Y, Park Y, Dellsperger KC, Kanaley JA. Acute aerobic exercise differentially alters acylated ghrelin and perceived fullness in normal-weight and obese individuals. J Appl Physiol. 2013;115(5):680-7.

6) Kanaley JA, Heden TD, Liu Y, W hale-Connell AT, Chockalingam A, Dellsperger KC, Fairchild TJ. Short-term aerobic exercise training increases postprandial pancreatic polypeptide but not peptide YY concentrations in obese individuals. Int J Obes (Lond). 2014;38(2):266-71.

7) Heden TD, Liu Y, Kearney ML, Park Y, Dellsperger KC, Thomas TR, Kanaley JA. Prior exercise and postprandial incretin responses in lean and obese individuals. Med Sci Sport Exer. 2013;45(10):1897-905.

8 ) Heden TD, Liu Y, Sims LJ, Kearney ML, W haley-Connell AT, Chockalingam A, Dellsperger KC, Fairchild TJ, Kanaley JA. Liquid meal composition, postprandial satiety hormones, and perceived appetite and satiety in obese women during acute caloric restriction. Eur $\mathrm{J}$ Endocrinol. 2013;168(4):593-600.

9) Heden TD, Liu Y, Sims LJ, W haley-Connell AT, Chockalingam A, Dellsperger KC, Kanaley JA. Meal frequency differentially alters postprandial triacylglycerol and insulin concentrations in obese women. Obesity (Silver Spring). 2013;21(1):123-9.

10) Heden TD, LeCheminant JD, Smith JD. Influence of weight classification on walking and jogging energy expenditure prediction in women. Res Q Exerc Sport. 2012;83(3):391-9.

11 ) Heden TD, Lox C, Rose P, Reid S, Kirk EP. One-set resistance training elevates energy expenditure for $72 \mathrm{~h}$ similar to three sets. Eur J Appl Physiol. 2011;111(3):477-484.

12) LeCheminant JD, Smith JD, Covington NK, Hardin-Renschen $T$, Heden TD. Pedometer use in university freshman: a randomized controlled pilot study. Am J Health Behav. 2011 35(6):777-784. 
13 ) LeCheminant JD, Covington NK, Smith J, Lox CL, Kirk EP, Heden TD. Evaluation of a University-Based Community Outreach W eight Management Program. Popul Health Manag. 2011 14(4):167-73.

14) LeCheminant JD, Heden TD, Smith JD, Covington NK. Comparison of energy expenditure, economy of movement, and pedometer counts among lean and overweight or obese women during physical activity. Eur J Appl Physiol. 2009;106(5):675-682.

15 ) Heden TD, Shepard S, Smith JD, Covington NK, LeCheminant JD. Resulting shifts in percentile and standard placements after comparison of the BOD POD and DXA. Int J Exercise Sci. 20081 (3).

\section{MANUSCRIPTS UNDER REVIEW OR IN PREPARATION}

1 ) Heden TD, Liu Y, Park Y, Nyhoff LM, Winn NC, Kanaley JA. Low-intensity physical activity reduces postprandial insulin secretion in obese adolescents consuming high- fructose or high-glucose diets. Under review in Journal of Physical Activity and Health.

2) Park Y, Heden TD, Liu Y, Nyhoff LM, Thyfault JP, Leidy HJ, Kanaley JA. Differential response of circulating GLP-1 and GIP to high protein and high carbohydrate meals: the second meal phenomena. In preparation, will be submitted to journal "Diabetologia".

3) Heden TD, Winn NC, Mari A, Booth FW, Rector RS, Thyfault JP, Kanaley JP. Post-dinner resistance exercise improves postprandial metabolic risk factors better than pre-dinner resistance exercise in obese patients with type 2 diabetes. In preparation, will be submitted to journal "Diabetologia".

4) Winn NC, Liu Y, Heden TD, Boyle LJ, Thyfault JP, Kanaley JP. Acute and chronic aerobic exercise training does not modulate irisin levels in obese individuals. In preparation.

\section{SELECTED PUBLISHED ABSTRACTS}

1) Heden TD, Liu Y, Park Y, Nyhoff LM, Winn NC, Kanaley JA. Low-intensity physical activity reduces postprandial insulin secretion in obese adolescents consuming high- fructose or high-glucose diets. Medicine and Science in Sports and Exercise, Volume 46:5 Supplement, 2014.

2 ) Kanaley JA, Park Y, Liu Y, Nyhoff L, W inn N, McCleery J, Heden TD. Can meal composition alter overnight glucose and insulin responses in obese individuals with impaired fasting glucose levels? Obesity Society. 2013. 
3) Heden TD, Liu Y, Kearney ML, Dellsperger KC, Thomas TR, Kanaley JA. Prior aerobic exercise and the incretin effect in normal-weight and obese individuals. Medicine and Science in Sports and Exercise, 45(5) Supplement, 2013.

4) Heden TD, Morris EM, Kearney ML, Liu T, Booth FW, Kanaley JA, Thyfault JP. Postprandial hepatic triacylglycerol secretion and fatty acid oxidation are not altered by prior aerobic exercise in obese-susceptible SpragueDawley rats. American Physiological Society Intersociety Meeting: Integrative Biology of Exercise, 21.10, 2012.

5) Heden TD, Liu Y, Kearney ML, Sims LJ, Dellsperger KC, WhaleyConnell AT, Chockalingam A, Nyhoff LM, Thomas MH, Kanaley JA. Brisk walking alters postprandial lipemia after fructose ingestion differently in normal-weight and obese individuals. Medicine and Science in Sports and Exercise 44(5):620, 2012.

6) Kanaley JA, Heden TD, Liu Y, Sims LJ, W haley-Connell AT, Chockalingam A, Dellsperger KC, Fairchild TJ. Pancreatic and gut peptide responses to short term exercise training in obese individuals. Obesity Society Annual Meeting, 2012.

7) Heden TD, Liu Y, Sims LJ, W haley-Connell AT, Chockalingam A, Kearney ML, Kanaley JA. Influence of meal frequency on postprandial glycemia and lipemia in obese individuals. Medicine and Science in Sports and Exercise 43(5):267, 2011.

8) Kanaley JA, Heden TD, Liu Y, Sims LJ, W haley-Connell AT, Chockalingam A, Kearney ML. Effect of meal composition and exercise training on glucose and insulin excursions over $12 \mathrm{~h}$. Medicine and Science in Sports and Exercise 43(5):102, 2011.

9) Kanaley JA, Heden TD, Liu Y, Sims LJ, W haley-Connell AT, Chockalingam A, Kearney ML. The second meal effect is altered by meal composition but not aerobic exercise training. Obesity Society Annual Meeting, 2011.

10 ) Heden TD, Kirk EP, Wessling R, Kanaley JA. Effect of resistance training dose on metabolic responses and behavior habits, 2010 Medicine and Science in Sports and Exercise 42(5):506, 2010. 


\section{ORAL PRESENTATIONS}

"Diet and exercise in gut and pancreatic hormone biology."

University of Missouri Nutrition and Exercise Physiology Spring Seminar Series, March,

2013.

"Prior aerobic exercise and the incretin effect in normal-weight and obese individuals." Central States American College of Sports Medicine, Columbia, MO, October, 2012.

\section{RESEARCH SUPPORT}

\section{Completed support:}

Agency: National Institute of Health

Title of Grant: T32 Training Grant, Exercise and Health: Integration from Molecule to Patient.

Date of Award: 9/2013-6/2014

Role: Trainee

Agency: MU Institute for Clinical and Translational Science

Title of Project: Molecular mechanisms of aerobic exercise induced alterations in hepatic fructose metabolism.

Date of Award: 8/2011 - 8/2012

Role: PI

Project Goals: To determine the underlying mechanisms by which exercise reduces postprandial lipemia in humans and rats fed high fructose diets.

Agency: SIUE Research Grants for Graduate Students

Title of Project: Influence of resistance training dose on resting energy expenditure.

Date of Award: 5/2008-5/2009

Role: PI

Project Goals: To determine how 1 set and 3 sets of whole body resistance training effects resting energy expenditure 24, 48, and 72 hours after the exercise bout in humans. 


\section{REFERENCES}

Jill A. Kanaley, Ph.D.

Professor and Associate Chair

Department of Nutrition and Exercise Physiology

University of Missouri - Columbia

Phone: (315) 744-3659

Email: kanaleyj@missouri.edu

John P. Thyfault, Ph.D.

Associate Professor

Department of Nutrition and Exercise Physiology

Internal Medicine

Veterans Affairs

University of Missouri - Columbia

Phone: (573) 882-9818

Email: thyfaultj@health.missouri.edu

John D. Smith, Ph.D.

Associate Professor

Department of Curriculum and Kinesiology

Texas A\&M University in San Antonio

Phone: (210) 784-2536

Email: john.smith@tamusa.tamus.edu 
Timothy Daniel Heden was born in Chicago, Illinois. After graduating from Oak Forest High School in Oak Forest, Illinois, he entered Southern Illinois University in Edwardsville, Illinois. He received a Bachelor of Science degree and a Master of Science in Education degree in Kinesiology during the Spring and Summer of 2007 and 2009, respectfully, at Southern Illinois University. In the Fall of 2009, he entered graduate school at the University of Missouri in Columbia where he obtained a Ph.D. degree in Exercise Physiology. 